\title{
All-sky visible and near infrared space astrometry
}

\author{
David Hobbs ${ }^{1}$ (D) . Anthony Brown ${ }^{2}$ - Erik Høg ${ }^{3}$. Carme Jordi ${ }^{4}$. \\ Daisuke Kawata ${ }^{5}$. Paolo Tanga ${ }^{6}$.Sergei Klioner $^{7}$. Alessandro Sozzetti ${ }^{8}$.

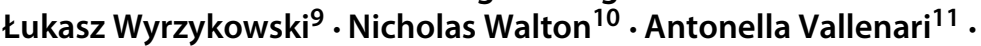 \\ Valeri Makarov $^{12}$. Jan Rybizki ${ }^{13}$. Fran Jiménez-Esteban ${ }^{14}$. José A. Caballero ${ }^{14}$. \\ Paul J. McMillan ${ }^{1}$ - Nathan Secrest ${ }^{12}$. Roger Mor ${ }^{4}$. Jeff J. Andrews ${ }^{3}$. \\ Tomaž Zwitter $^{15}$. Cristina Chiappini ${ }^{16}$. Johan P. U. Fynbo ${ }^{3}$. Yuan-Sen Ting ${ }^{17}$. \\ Daniel Hestroffer ${ }^{18}$. Lennart Lindegren ${ }^{1}$ - Barbara McArthur ${ }^{19}$. \\ Naoteru Gouda ${ }^{20}$. Anna Moore ${ }^{21}$. Oscar A. Gonzalez ${ }^{22}$ - Mattia Vaccari23,24
}

Received: 24 July 2020 / Accepted: 28 January 2021 / Published online: 11 March 2021

(C) The Author(s) 2021

\begin{abstract}
The era of all-sky space astrometry began with the Hipparcos mission in 1989 and provided the first very accurate catalogue of apparent magnitudes, positions, parallaxes and proper motions of 120000 bright stars at the milliarcsec (or milliarcsec per year) accuracy level. Hipparcos has now been superseded by the results of the Gaia mission. The second Gaia data release contained astrometric data for almost 1.7 billion sources with tens of microarcsec (or microarcsec per year) accuracy in a vast volume of the Milky Way and future data releases will further improve on this. Gaia has just completed its nominal 5-year mission (July 2019), but is expected to continue in operations for an extended period of an additional 5 years through to mid 2024. Its final catalogue to be released $\sim 2027$, will provide astrometry for $\sim 2$ billion sources, with astrometric precisions reaching 10 microarcsec. Why is accurate astrometry so important? The answer is that it provides fundamental data which underpin much of modern observational astronomy as will be detailed in this White Paper. All-sky visible and Near-InfraRed (NIR) astrometry with a wavelength cutoff in the K-band is not just focused on a single or small number of key science cases. Instead, it is extremely broad, answering key science questions in nearly every branch of astronomy while also providing a dense and accurate visible-NIR reference frame needed for future astronomy facilities.
\end{abstract}

Keywords Space astrometry · Galactic dynamics · Space mission · Photometry • Gaia

David Hobbs

david@astro.lu.se

Extended author information available on the last page of the article. 


\section{Introduction}

For almost 2 billion common stars the combination of two all-sky space observatories would provide an astrometric foundation for all branches of astronomy - from the Solar System and stellar systems, including exoplanet systems, to compact galaxies, quasars, neutron stars, binaries and dark matter (DM) substructures. The addition of NIR will result in up to 8 billion newly measured stars in some of the most obscured parts of our Galaxy, and crucially reveal the very heart of the Galactic bulge region (see Fig. 1 for a note of caution!).

In this White Paper we argue that rather than improving on the accuracy to answer specific science questions, a greater overall science return can be achieved by going deeper than Gaia and by expanding the wavelength range to the NIR. An obvious question to ask is - can a more accurate all-sky mission than Gaia be done? Clearly the answer is yes, if we can build a space telescope with a larger aperture $(D)$ but in practice it is very difficult to do this without greatly inflating the cost of the mission. Gaia was designed very well and only just fitted in the available launchers so improving the telescope's angular resolution (i.e. minimum angular separation) $R \propto \lambda / D$ at a fixed wavelength $\lambda$ is very costly. Other mission proposals have tried to avoid this by employing long focal lengths and advanced metrology systems for ultra-accurate narrow field proposals, like SIM, NEAT and Theia, but these missions were focused on answering important specific science cases and did not aim to do a broad all-sky astrometric survey. Nevertheless, the metrology systems explored may find application in improving a future all-sky mission.

A new all-sky NIR astrometric mission will expand and improve on the science cases of Gaia using basic astrometry. Key topics are focused on what dark matter is and how is it distributed, how the Milky Way was formed and how has it been impacted by mergers and collisions? How do stars form and how does stellar feedback affect star formation; what are the properties of stars, particularly those shrouded in dust, and small Solar System bodies; how are they distributed and what is their motion? How many co-planar systems like ours (with Earth-sized and giant planets) are there and what fraction have planets with long period orbits? To answer these questions there are three main science challenges for a new all-sky astrometry mission:

1. NIR astrometry (and simultaneous photometry) is crucial for penetrating obscured regions and for observing intrinsically red objects when implemented with sufficient accuracy. Peering through the dust of the Milky Way to obtain a dense sampling of the phase space is necessary to properly study the bulge, bar, bar-disc interface and spiral arms. Spiral structure can excite stars to radially migrate and induce disc heating and accurate measurements of the 3-D motion and properties of these obscured stars are needed to trace the dynamical history and evolution of our Galaxy. Preliminary estimates show that a new NIR astrometry mission would observe at least 5 times as many stars as Gaia, assuming the same magnitude range, giving a huge increase in the catalogue size and would dramatically increased phase space sampling of the disc, especially of the innermost regions where co-existing populations require better statistics. 

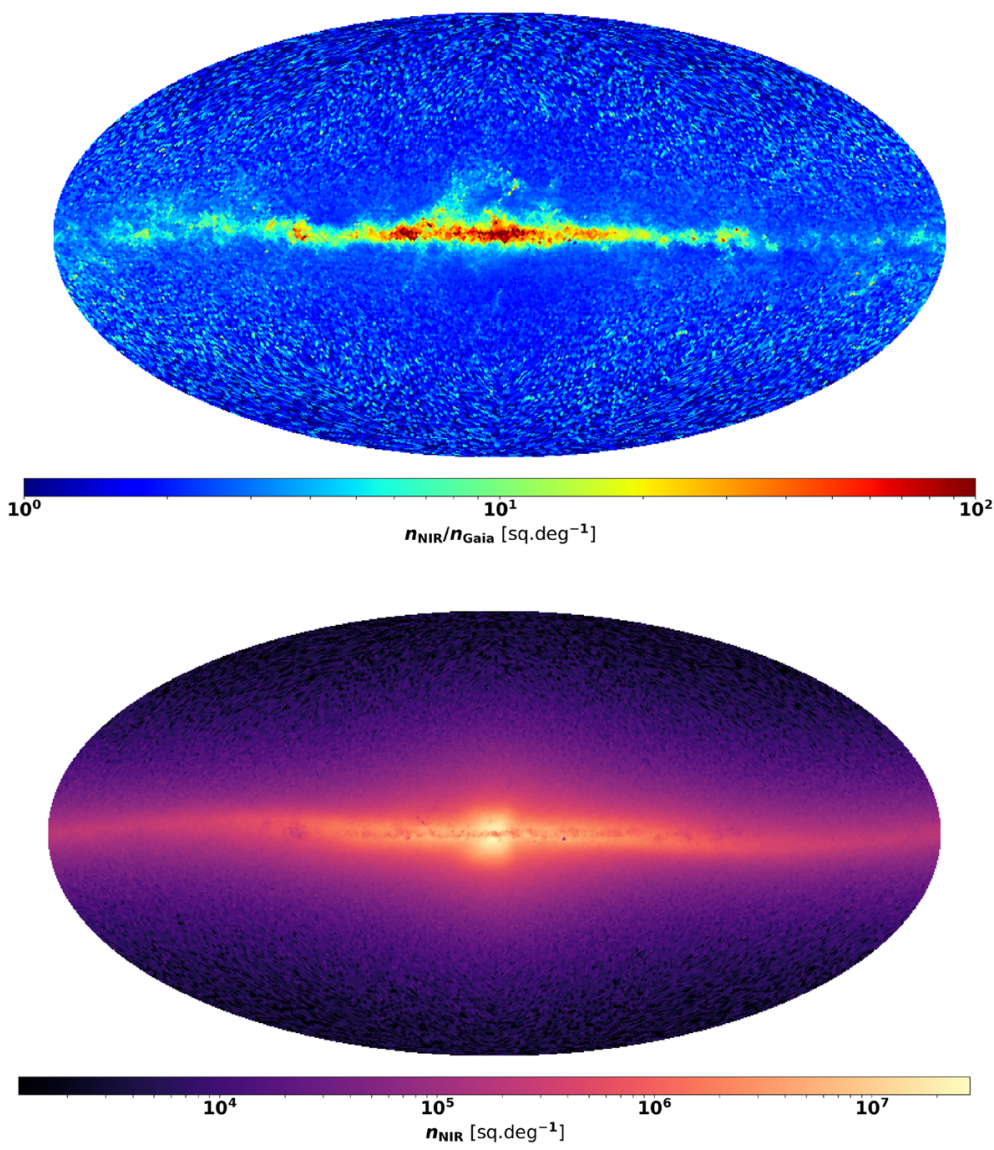

Fig. 1 All-sky projection in Galactic coordinates of the star count ratio per square degree between GaiaNIR and Gaia (G-band limit of 20.7th mag giving 1.5 billion Gaia sources). In total 5 times more stars could be observed, especially in the disc where extinction is highest, by GaiaNIR for the H-band limit of 20th mag (top figure) and 6 times more stars could be observed by including the K-band limit of 20th mag. Crowding is not taken into account here and will limit the increase in numbers in the densest areas. In the bottom figure we show the corresponding H-band number densities. The underlying Milky Way model (which does not include clusters or external galaxies, e.g. SMC and LMC) is similar to GDR2mock [204] (using Galaxia [212] with the extinction map of [34]) but only $0.1 \%$ of the stars are sampled explaining the noise in low density regions. A note of caution, the estimation of the star count ratio between Gaia and GaiaNIR is uncertain due to the uncertainty in the extinction model used (older models gave a lower ratio of around 3), mainly towards the centre of the Galaxy. However, one could argue that this uncertainty is a key science case in itself that cannot be resolved by Gaia alone

2. A new mission could be combined with the older Gaia catalogue (currently $\sim 1.7$ billion sources) with a 20 year interval to give a much longer baseline of 2535 years, with very accurate proper motions (a factor of 14-20 better in the two components) and improved parallaxes needed to measure larger distances. Dynamical studies in all of the outer halo would give an enormous sample and greatly enhance the resolving of tangential motions in streams and local dwarf 
galaxies, with a potential accuracy of $2-3 \mathrm{~km} \mathrm{~s}^{-1}$ for samples out to $\sim 100 \mathrm{kpc}$. This will provide great insight into the gravitational potential in the outer reaches of the Milky Way where halo streams are sensitive probes. Proper motions will also allow the study of massive local group galaxies (for instance Cen-A). At the same time the parallaxes, especially of binaries, will be much improved when astrometric data from two missions are combined and the detection of planets with significantly longer periods than by Gaia alone can be achieved. A significant population of stars with planetary system architectures similar to our Sun's (i.e. long period, massive gas giants, like Jupiter and Saturn, in the outer reaches, shielding Earth type planets in the star's habitable zone) will be discovered.

3. A new mission would allow the slowly degrading accuracy of the Gaia visible reference frame, which will become the fundamental Celestial Reference Frame and the basis for all modern astronomical measurements, to be re-initialised back to a maximal precision. This degradation is due to errors in its spin and due to small proper motion patterns which are not accounted for. The catalogue accuracy itself will decay more rapidly due to errors in the measured proper motions. Dense and accurate reference grids are needed for forthcoming giant telescopes but also for smaller instruments currently operating or being planned. The extension of this visible reference frame into the NIR is an important step given that so many new space and ground based observatories will have infrared sensitive instruments. A new mission would provide better accuracy to explore proper motion patterns (e.g. from the Sun's Galactic acceleration and gravitational waves), real time cosmology and fundamental physics.

In summary, the new mission proposed here will observe many new stars in obscured regions. We estimate at least 5 times as many stars will be observed, giving up to 8 billion new objects. NIR opens up a new wavelength range which allows us to probe the dusty obscured regions of the Galactic disc with high-precision astrometry and broad-band high-resolution photometry, while out of the Galactic plane a new mission will go deeper to enhance the halo science cases and provide complementary legacy data to ground based surveys such as the Rubin Observatory. A common astrometric solution for the two missions will give greatly improved proper motions but also improve the parallaxes, for up to 2 billion common stars. Long term maintenance and expansion of the dense and very accurate celestial reference frame with a new mission is necessary for future precise astronomical observations and provides an essential service for the astronomical community. These features ensure that a new mission is not simply an increment on the previous one but will create an astrometric revolution in itself!

ESA's study of the proposal for GaiaNIR [108] concluded that the overall cost of the mission would be similar to that of Gaia. However, as the US is the world leader in detector technology we recently proposed [109, 154] a collaboration with the US on this project, particularly for the detectors, which would make the mission feasible and allow it to remain within the ESA M-class mission budget provided the US, Japanese, and Australian partners contribute significantly. 


\section{Science case background}

The nominal Gaia mission [87] will provide global astrometry to unprecedented accuracies (17-25 $\mu \mathrm{as}\left(\mathrm{yr}^{-1}\right)$ at $\left.G=15\right)$, in positions, absolute parallaxes and proper motions, with the addition of all-sky homogeneous multi-colour photometry and spectroscopy. These unique capabilities go well beyond and are complementary to the science cases being addressed by ground-based surveys (such as RAVE, SDSS (photometry, SEGUE and APOGEE), Gaia-ESO, LAMOST, GALAH, VISTA (VVV), Pan-STARRS, DES, the Rubin Observatory, WEAVE and 4MOST; see Section 7 on synergies). A space-based mission avoids the limitations caused by the turbulent atmosphere and the use of Earth rotation parameters and models of nutation and precession. All-sky space-based astrometry leads to a global solution and provides a rigid sphere for a celestial reference frame that cannot be accurately obtained with any other method [89].

An obvious technological improvement to the current Gaia mission is to also go into the non-thermal Near-InfraRed (NIR) with a wavelength cutoff in the K-band allowing the new mission to probe deeper through the Galactic dust to observe the structure and kinematics of the star-forming regions in the disc, the spiral arms and the bulge region, to give model independent distances and proper motions in these obscured parts of the sky. Additionally, having two 5 or 10-year Gaia-like missions separated by 20 years would give 14-20 times better proper motions for a few billion common stars and also improved parallax determinations with new observations. After the publication of the final Gaia catalogue the positions of stars will be accurately known at the chosen reference epoch, which will be close to mid 2019. However, this accurate positional information and the accuracy of the link to the VLBI reference frame will slowly degrade due to the small uncertainties in the proper motions of the stars. Hence, it is very desirable to repeat the measurements of Gaia after about 20 years to maintain the positional accuracy of the stars and the visible reference frame. Gaia is already one of the most transformational missions ever as measured in terms of scientific output. The rate at which papers appear using Gaia data is soon to outstrip the HST and a new NIR mission will enable a further explosion of science insight.

Hobbs et al. [108] proposed to the European Space Agency (ESA) a new all-sky NIR astrometry mission, called GaiaNIR. Such a NIR space observatory is however not possible today: it requires new types of Time Delay Integration (TDI) NIR detectors to scan the entire sky and to measure global absolute parallaxes. Although developing TDI-NIR detectors is a significant challenge, the US is well placed to advance such technology, and thus to open the doors to what can become the first international collaboration for a global astrometry space observatory. McArthur et al. [154] and Hobbs et al. [109] submitted White Papers to the US decadal survey (ASTRO 2020) outlining the science cases and a possible US-European collaboration. The science is also being pursued elsewhere, for example, the Australian National University is developing NIR astronomical detector technology with TDI capabilities. ISAS/JAXA have selected small-JASMINE for their M-3 mission with a 
current scheduled launch in the mid-2020s, to do relative (to Gaia) astrometry in the NIR, but only focusing on the small region within $\sim 100 \mathrm{pc}$ from the Galactic Centre and relatively bright $(\mathrm{Hw}<15 \mathrm{mag})$ stars. In Section 8 we will return to the possible implementation of our mission.

Below we present the science cases for a new all-sky astrometry mission assuming the associated technological challenges can be resolved in reasonable time. The accuracy of the new mission should be at least that of Gaia using tried and trusted instrumentation, techniques, and lessons learned from Gaia to unveil a wealth of new and more accurate information about our Galaxy. The science cases have been roughly divided into three Sections 1) NIR science cases; 2) improved proper motion science cases and 3) reference frame science cases. Clearly there is a strong overlap between these three areas but they are presented separately for clarity.

\subsection{Measurement concept}

The measurement concept for obtaining global astrometric measurements only possible from space is well-defined and well demonstrated (see, for example, $[146,181]$ ). Indeed, the concept is identical to that of the currently flying Gaia mission where the nominal mission lifetime is 5-6 years. However, for Gaia, the community has already proposed to continue observations for a total of 10 years if fuel consumption and the hardware on-board continue to operate as expected. Such a mission will effectively double the number of measurements giving an accuracy improvement by a factor of $\sqrt{2}$ in the positions and parallaxes but a factor of $2 \sqrt{2}$ in the proper motions which also benefit from a doubling of the measurement baseline. However, in addition to just doubling the number of measurements, if we also add a gap between missions, a simple calculation shows that the combination of two 5-year missions (labelled with subscript $\mathrm{N}$ for GaiaNIR and $\mathrm{G}$ for Gaia), assuming a positional and a proper motion accuracy of $25 \mu \mathrm{as}\left(\mathrm{yr}^{-1}\right)^{1}$ with a separation of 20 years will give (see equation 2 in [85]):

$$
\begin{gathered}
\sigma_{\mu_{\alpha} *}=\frac{\sqrt{\sigma_{\alpha_{\mathrm{N}}^{*}}^{2}+\sigma_{\alpha_{\mathrm{G}}^{*}}^{2}}}{t_{\mathrm{N}}-t_{\mathrm{G}}}=\frac{\sqrt{25^{2}+25^{2}}}{20} \sim 1.77 \mu \mathrm{as} \mathrm{yr}^{-1}, \\
\sigma_{\mu_{\delta}}=\frac{\sqrt{\sigma_{\delta_{\mathrm{N}}}^{2}+\sigma_{\delta_{\mathrm{G}}}^{2}}}{t_{\mathrm{N}}-t_{\mathrm{G}}}=\frac{\sqrt{25^{2}+25^{2}}}{20} \sim 1.77 \mu \mathrm{as} \mathrm{yr}^{-1}
\end{gathered}
$$

which is a factor of 14 better in both proper motion components. If we then assume two 10-year missions one gets an extra factor of $\sqrt{2}$ improvement in the individual positions from each mission giving an overall improvement by a factor of 20 for both

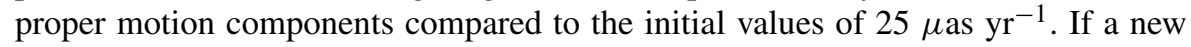
mission follows we would get these improvements in proper motions for a few billion stars. Parallaxes will also improve mainly due to the additional measurements [110]

\footnotetext{
${ }^{1}$ This is the assumed end of mission (5 years) accuracy but neglects the fact that proper motions are generally better than positions. On the other hand, we do not account for parallax improvements which indirectly improve the joint proper motion solutions. Our AGISLab simulations, discussed above, include all of these effects naturally and the results are roughly in agreement with these simplistic predictions.
} 
but also because the proper motions are much more accurate. The parallaxes can then be better determined (an indirect improvement) which has already been demonstrated in the TGAS solution [159, 160] for Gaia where Hipparcos/Tycho-2 data were combined with Gaia's to form the first data release. Residual systematic errors will be present in both Gaia and the new mission, but they should be uncorrelated and it may be possible to use a joint solution of both missions to partially reduce these errors.

AGISLab numerical simulations (See Fig. 6 [108]) of two joint missions using periods of 5 and 10 years for each mission segment, respectively, show that, indeed, the improvements in the final astrometric errors for proper motion are roughly factors of 14 and 20 better than a 5-year Gaia mission alone. Likewise, the improvements in positions and parallax followed the prediction of $\sqrt{2}$ also mentioned above. The numerical simulations show that these simplistic estimates give the correct order of improvement for 'real' astrometric missions. This approach brings the advantage of a largely known and already implemented mission concept [145], which can be improved based on existing experience (particularly by going into the NIR). The lessons learned from Gaia will be invaluable and improvements in the data processing and instrument modelling can be built on already well developed concepts. To accomplish this goal we need to build a new all-sky astrometry mission to fly around 2040 .

\section{NIR astrometric science cases}

\subsection{The bulge/bar-disc interface and radial migration}

Since Gaia was proposed, it has become clear that the evolution of the Milky Way is far more complex than had been realized. Not only is it not in equilibrium (e.g. [12]), but its stars move away from their birth places, a process called radial migration. Originally it was proposed that radial migration could be triggered by bars (e.g. [83]) or spiral arms [211]. Subsequent studies have shown the migration efficiency to vary with time and distance from the Galactic Centre, and to be strongly influenced by the bar and its interaction with spiral arms [40, 163] as well as by minor mergers (e.g. [27]). This has important consequences for the chemodynamical evolution of the Galaxy [164, 165, 203, 209]. It implies that even the local volume near the Sun cannot be understood in isolation, without a proper description of the innermost regions of the Milky Way and its merger history.

An important element of radial migration happens at the interface between the bar and the spiral arms, which occurs in the inner few kpc of the Galaxy. [100] demonstrated that radial migration can be captured as systematically different stellar motions by about $10 \mathrm{~km} \mathrm{~s}^{-1}$ at different sides of the spiral arm. It also needs to be tested at a large range of radii, because it is expected to be strong at the resonances [211] and the resonance radii of the bar and spiral arms are unknown in the Milky Way. Therefore, it is required to trace the kinematics of stars on both sides of the spiral arm at a large range of radius from the bar to the outer disc, i.e. galactocentric radius range of $0<R_{\mathrm{G}} \lesssim 10 \mathrm{kpc}$. Also, radial migration is efficient for kinematically colder stars [219] which can leave imprints in some of the observed chemodynamical 
relations [166], and hence it is crucial to trace stellar kinematics (to be complemented by chemical information from ground based facilities) close to the Galactic disc midplane. Gaia has sufficient astrometric accuracy to observe such motions, especially when traced with bright stars such as Cepheids [181]. However, in the inner disc and the far side of the spiral arms extinction is a serious problem, and studies at visible wavelengths, such as with Gaia, are unable to see through the dust to observe any but the intrinsically brightest and/or nearest stars. A new NIR astrometry mission would allow us to probe this region and determine proper motions and parallaxes of the stars there. This is the only way that we will be able to get a full $3 \mathrm{D}$ picture of the dynamics of this vitally important region of the Galaxy (see [28], for a review).

\subsection{The bulge/bar}

The centre of the Galaxy is dominated by a stellar bar, which has created a peanutshaped pseudo-bulge [174, 252]. There are also claims that the bar extends as far as $5 \mathrm{kpc}$ from the Galactic Centre in the plane of the Galaxy [245]. Prior to Gaia DR2, dynamical studies of the bar (e.g. [187]) were typically limited to using line-of-sight velocities for stars in a relatively small number of fields. For a review on the bulge/bar before GaiaDR2 see [19]. Progress has been made since Gaia DR2 by using Gaia positions and proper motions to tie the relative proper motions found for stars in the VVV Infrared Astrometric Catalogue (VIRAC) as found by [218] to an absolute rest frame. Using these proper motions it has been possible to investigate the kinematics of the bar [58, 208], and even to measure the pattern speed of its rotation [207]. These studies are still hampered by the relatively low precision of VIRAC proper motions, and the difficulty of relating them to the absolute rest frame. The pattern speed of the bar is also measured with the combination of Gaia DR2 and APOGEE by [35]. However, their study is also not free from systematics due to challenges of obtaining accurate photometric distance and small number of Gaia stars in the disc plane. Indeed, when combining Gaia parallax information with APOGEE spectroscopic information plus other photometric bands, the Bayesian code StarHorse [191] is able to obtain distances in the bar/bulge area which still show uncertainties of the order of $1 \mathrm{kpc}$ ( 10\% precision) which is clearly not enough to disentangle the mix of populations in the the bulge area [192]. A new NIR astrometry mission would directly provide proper motions and parallaxes for stars in this complicated region and allow us to disentangle its dynamics. For example, it would allow us to learn about the growth of the bar by observing stars that it recently captured [14] and to what extent a spheroidal component is also present. Astrometric mapping of the Galactic plane would allow us to answer once and for all critical questions regarding the structure of the inner Milky Way: is there a large scale, in-plane inner disc or ring [63]; what is the nature of the density and velocity dispersion peak in the central degree of the Galaxy [240]; and how does the apparent multiple component nature of the bulge extend to the innermost regions [19]?

Cosmological models find that the density profiles of dark matter haloes peak at their centre with $\rho \propto r^{-1}$. However, this is in simulations assuming that dark matter is dynamically cold, and without any of the complicated physics associated 
with the baryonic components of galaxies. It has been shown that feedback from star formation can affect the dark matter density profile [98], and the effect of the bar on the dark matter halo is also the subject of debate [112].

The Milky Way presents our best opportunity to study the dark matter content near the centre of an $L^{*}$ galaxy [59], which may teach us about the nature of dark matter and/or the processes associated with galaxy formation and evolution on these scales. Without an astrometric mission that operates in the NIR, proper motions for these stars will never be determined, and the unique opportunity we have to study all-sky and the $6 \mathrm{D}$ phase space distribution of stars in the centre of an $L^{*}$ galaxy will be squandered. However, many of these objects will not have been observed by Gaia so that a new NIR mission will give unique measurements of them with similar accuracy to Gaia. The parallaxes, and proper motions at the Galactic Centre distances will thus not be very accurate for many new single objects. However, double epoch observations for bright stars will give a smaller sample of very accurate parallaxes and proper motions. Most ground-based IR surveys, for example GRAVITY [73], will cover very small patches on the sky compared to the very unique all-sky astrometry that a new NIR mission can offer. While GRAVITY will be crucial to investigate better the orbits of stars around the supermassive black hole at the Galactic Centre, a new NIR astrometry mission will constrain the very detailed dynamical and orbital structure of many more stars and at greater distances around the black hole (i.e. at larger scales).

\subsection{The milky way disc}

Gaia data has uncovered a wealth of unexpected structure and disequilibrium in the disc of the Milky Way [13, 88, 124], which has changed how we view it as a system. It is no longer sufficient to approximate it as an equilibrium structure even in the solar neighbourhood (e.g. [182]), and we are forced to consider the fact that the local velocity field is rich in structure [88]. This includes a 'phase-spiral' when looking at the vertical velocity as a function of height above the Galactic plane [13], thought to be the consequence of the passage of a dwarf galaxy through the plane of the Milky Way. Disequilibrium associated with the Milky Way's spiral structure affects the velocity field of the disc stars in a way that varies substantially across the Galaxy. The Galactic plane itself shows clear signs of a warp [84, 186]. This means that approximations such as thinking of the Galaxy as having a simple rotation curve which is a function of radius must be discarded, and we must map the velocity field across the Galactic plane. A future astrometric mission will allow us to do this far more accurately than Gaia alone, because of the improved proper motions, and working in the infrared will alleviate the selection effects caused by dust extinction which mean that stars in the midplane of the Galaxy are not seen by Gaia. For example, the phase spiral feature has a scale of $0.1 \mathrm{kpc}$ in distance and a few $\mathrm{km} \mathrm{s}^{-1}$ in velocity. To identify the origin of such phase spiral features, requires this feature to be measured at large radial $\left(0<R_{\mathrm{G}}<\sim 20 \mathrm{kpc}\right)$ and azimuthal angle $\left(\theta< \pm 60^{\circ}\right)$ ranges with a similar accuracy and number of stars to those currently achieved with Gaia DR2 in the solar neighbourhood. This will enable us to measure the vertical and 
in-plane oscillation of the Galactic disc in a large radial range, which will be crucial information for Galactoseismology, to examine the influences of the satellite galaxies interactions and the bar formation as well as measuring the stellar disc and dark matter density (e.g. [120]).

\subsection{The spiral arms}

It is surprising that very little is known about the spiral structure of the Milky Way (e.g. [241, 242]). The Bar and Spiral Structure Legacy (BeSSeL) survey ${ }^{2}$ and the Japanese VLBI Exploration of Radio Astrometry (VERA) survey ${ }^{3}$ have yielded over 100 parallax measurements in the spiral arms and the central bar with typical parallax accuracy of about $\pm 20 \mu \mathrm{as}$, and some as good as $\pm 5 \mu$ as. These radio measurements are providing good constraints on the fundamental parameters of the Galaxy, including the distance to the Galactic Centre. Gaia on the other hand is very limited by extinction at visible wavelengths and will not be able to freely probe the Galactic plane [197].

The Milky Way is the only galaxy in which we can accurately observe the motion of the stars inside and around the spiral arms. This provides the crucial information for the origin of the spiral arms, which is one of the fundamental questions in galactic astronomy, but still hotly debated. The classical density wave theory [144] is recently challenged by transient spiral arm scenarios seen in N-body simulations (e.g. [210]). Using Gaia DR1, [15] studied the stellar motions in the Perseus arm using 77 Cepheids, and found $\sim 10 \mathrm{~km} \mathrm{~s}^{-1}$ level of velocity structures and a tentative sign of the disruption of the Perseus arm. Firmly testing the spiral arm scenario requires measurements of the stellar motion inside and around the different spiral arms at different radii with many more tracer samples with a few $\mathrm{km} \mathrm{s}^{-1}$ accuracy in velocity and sub kpc accuracy in distance, to resolve the motion at the different sides of the spiral arms. A new NIR astrometry mission can provide many more samples of stars in the disc plane with enough astrometric accuracy up to about the distance of $8 \mathrm{kpc}$, and can uncover the stellar motion around the Outer, Perseus, Local, Sagittarius, and Scutum-Centaurus arms over a large range of Galactocentric radii and azimuthal angles. This will ultimately provide an answer for the origin of the spiral arms.

A new NIR astrometry mission will provide a more complete sample of bright stars up to a larger distance, which will enable us to map the stellar density distribution and identify the over-density of stars for the nearby spiral arms, such as the Perseus, Local and the Sagittarius arms. [168] identified the stellar over-density of the Local arm, and found a tentative offset from the high-mass star-forming regions identified with VLBI. Measurements of such offsets for different spiral arms at different radii help to constrain the spiral arm scenario, and also answer which spiral arms in the Milky Way are the major stellar arms (e.g. [25]). In addition, the combination of the accurate density distribution and kinematic measurements for tracer populations

\footnotetext{
${ }^{2}$ See this http://bessel.vlbi-astrometry.org.

${ }^{3}$ See this http://veraserver.mtk.nao.ac.jp.
} 
allows us to measure the pattern speed of the spiral arm at different radii by applying the continuity equation locally $[158,235]$.

Spiral arms are the main areas of star formation in the Milky Way, and are responsible for a significant portion of radial migration and disc heating. A greater understanding of their structure will help in understanding these processes. A new NIR astrometry mission would be able to see through dust and would allow us to better map the spiral arms and the star-forming regions, not just for a few hundred objects as is the case for radio astrometry, but potentially for hundreds of millions of objects, revealing much more detailed structure that radio surveys cannot hope to achieve. A new NIR astrometry mission could provide model-independent distances and proper motions, avoiding the need to use extinction maps together with galaxy modelling but also better constraining extinction maps. Galactic archaeology relies on accurate distances and needs better accuracy towards the spiral arms and the Galactic Centre to give accurate stellar ages and astrophysical parameters in these important regions.

\subsection{Galactic rotation curve and dark matter}

Currently, the gravitational force at different points in the inner disc of the Milky Way is not well known. This property is key to learning about the distribution of dark (and luminous) matter near the centre of the Milky Way. A new NIR astrometry mission will be the only instrument able to solve this problem since it will unveil the inner dynamics of the disc, in unprecedented detail, from hundreds of millions of stars, in the bulge, bar, spiral arms, and between the spiral arms. This will allow us to perform high spatial resolution mapping of the dark matter distribution in these regions. This will be key to resolving questions regarding the nature of dark matter particles, by showing us whether the Galaxy has a cored or cusped dark matter halo, whether there are any thin, disc-like components to the dark matter distribution, and whether spiral arms have their own dark matter component.

In external galaxies, and the outer parts of our own (where we can assume axisymmetry), it is common to approximate the gravitational force in the plane using a rotation curve. In the inner Milky Way, this is not an appropriate approach because of the non-axisymmetry $[125,236]$ and because some key tracers $\left(\mathrm{H}_{\mathrm{I}}\right.$ or $\mathrm{CO}$ regions $)$ provide poor spatial coverage [54]. A more sophisticated approach is required (e.g. $[113,187])$ along with more data. VLBI cm measurements of masers [197] are excellent probes of the rotation curve, kinematics and structure in the low latitude regions of the Milky Way. Unfortunately they are too limited in the number of targets (at most a few hundreds when their survey is complete), and are only sensitive in starforming regions (thus only in the spiral arms or along the dust lanes of the bar), to provide high spatial resolution. VLBI mm measurements with ALMA will also play a role in a very near future to do similar science as cm-VLBI, likely with many more detections because there are more emission lines at $\mathrm{mm}$ - than at $\mathrm{cm}$-wavelengths. Gaia and VLBI will help in the quest to understand the inner dynamics of the Galaxy but a NIR capable astrometric mission would allow much greater insight.

The incredible strength of a new NIR astrometry mission with respect to radio interferometry is that it will not be restricted to any particular disc perturbations or 
regions and will observe millions of sources. A new mission will be the only way to probe both the low latitude arm and inter-arm regions, as well as directly inside the bar. It will improve greatly on $\mathrm{cm}$ - and mm-VLBI measurements restricted to star-forming regions only, and 21-cm HI or mm CO measurements which do not allow direct measurements of distances and proper motions of gas clouds. A new mission is the only way to determine hundreds of millions of velocities at all points in the Galactic plane and model their dynamics. [236] and [125] demonstrated that the rotation curve estimates from stellar kinematics are affected by non-axisymmetric structures, such as spiral arms, and it is important to measure the rotation curve at different azimuthal angles of the Galaxy. A new NIR astrometry mission will provide accurate enough positions and velocities for a large number of thin disc stars hidden in the dust as far as $\sim 8 \mathrm{kpc}$ and covers the azimuthal angle of $\theta< \pm 60^{\circ}$. This will make it possible to make a comparison with stellar rotation curves of other galaxies, and the cusp-core controversy (among others) will then be accurately investigated.

\subsection{Baryonic content of the Galaxy}

In Section 3.5 we described the role that a new NIR astrometry mission can play in the determination of the rotation curve of the Galaxy and the dark matter distribution. When using dynamics and kinematics to determine the dark matter distribution it is crucial to have a good knowledge of the distribution of the baryonic content of the Galaxy. The stellar initial mass function (IMF), the star formation history (SFH), the star formation rate (SFR), and the density laws are fundamental to determine the baryonic content of the Galaxy. The SFH contains essential information to understand the Galactic structure and evolution, including key information on its merger history (e.g. [93]). The SFH and the IMF directly influence the chemical composition of the Galaxy, together with the density laws they constitute a good description of the stellar content of the Galaxy.

Faint M dwarfs and brown dwarfs have an important contribution in the total stellar mass density of the Milky Way (e.g. [156]). A new NIR astrometry mission will be a key instrument to have a full-sky census of faint $M$ dwarfs and brown dwarfs. The photometry, the positions and the parallaxes of a new NIR astrometry mission will be very important to improve the determination of the IMF in the low-mass stars and sub-stellar regime. Complemented by Gaia data we will be able to cover a large mass range, from the fainter and reddest sub-stellar objects up to the more massive, bluer, and brighter stars, covering a wide range of the IMF. Furthermore, as a new NIR astrometry mission will survey plenty of old stars in the Galaxy, the photometry and astrometry for the full sky census of M dwarfs and brown dwarfs will reduce much of the Star Formation Rate (SFR) and Star Formation History (SFH) uncertainty for the oldest epochs (more than 7 Gyrs ago, e.g. [169]). Additionally, the positions and parallaxes of a new NIR astrometry mission will allow to determine the density distribution of brown dwarfs and the faintest $\mathrm{M}$ dwarfs (and pre-main sequence) much beyond the solar neighbourhood.

A determination of the SFR and the SFH together with the IMF and density distribution will give us the full picture of the distribution of the stellar content in the Milky Way, which is critical for the determination of the dark matter distribution. A 
model for the star formation efficiency and complementary observations can be used to estimate both the gas needed to form the stars through the history of the Milky Way and the present gas distribution. The caveat remains of the impact of radial migration on these estimates. Therefore, once again, it is crucial to trace the whole disc and innermost areas of the Galaxy in order to be able to model and estimate the impact of radial migration onto our estimates for the SFHs and how we interpret the chemokinematic-age relations in the Galaxy, and in particular in the disc (see discussion in [161] on how age can be used to solve some of the key Galactic archaeology open questions).

\subsection{An age map of the inner milky way}

Our understanding of the formation history of the inner regions of the Milky Way is hampered by our lack of knowledge of the large scale distribution of its relative stellar ages. This is a purely technical limitation. The magnitude of the Main Sequence Turn Off (MSTO) is the most age-sensitive tracer that allows, with a combination of optical and NIR photometry, to break the age-metallicity degeneracy in the colour-magnitude diagram to recover the star formation histories of the bulge stellar population (see for example [198]). Astrometric information is the most critical ingredient for the success of these studies, as proper-motions with precision $<0.3 \mathrm{mas} / \mathrm{yr}$ are required to separate bulge MSTO stars from the foreground disc. For this reason, bulge stellardating studies have been so far restricted to a few discrete fields with deep enough photometry (mostly with HST) to reach the magnitude of the MSTO. Most of them have been carried out at high Galactic latitudes to minimise the effects of reddening.

Plans for the Rubin Observatory have been suggested by the community to build on the legacy of Gaia and the VVV survey to perform this type of study across the bulge $[30,97]$. These fields are in very complicated areas for the Rubin Observatory due to the combined effects of crowding and faint magnitudes. Therefore, even in the best case scenario in which some of these vital inner Galactic areas are covered by the Rubin Observatory, its field-coverage would certainly be constrained to high Galactic latitudes $(|b|>2.5)$. A new NIR astrometry mission would break all of these restrictions, providing homogeneous proper-motions, parallaxes, and NIR magnitudes well within the required precision which, when coupled with the other multi-band surveys, would allow us to estimate accurate ages and metallicities for millions of stars across all the components of the inner Milky Way. These will constitute extremely valuable targets to be followed up by the Mosaic MOS at ELT and will also be complementary to the efforts being made in asteroseismology [161], which right now are still concentrated on the outer disc (e.g. [3, 4]).

\subsection{Clusters}

Stars are continuously formed in clusters of tens to thousands and evolve together for a shorter ( $\sim 100 \mathrm{Myr}$ ) or longer time (a few Gyr) in associations or open clusters, respectively, depending on whether they are gravitationally bound or unbound. Clusters are often located in the spiral arms of the Milky Way and are composed of young stars that have recently formed in the disc. The stars belonging to a cluster 
have roughly the same age and metallicity and can be used to probe the Galactic disc structure and formation rate, as well as to study young star properties and their formation process (see for example: [142]) as well as probing radial migration (see [4] and [50]).

More than 2000 open clusters are known today, most of them within a distance of $\sim 2 \mathrm{kpc}$ from the Sun [128]. This is roughly $1 \%$ of the total population of over $10^{5}$ open clusters expected in the Galaxy [170]. The Gaia mission is extending the census of open clusters, most of which are located at high Galactic latitudes with low interstellar extinction. More than 2000 open clusters were listed as such in the preGaia era [69, 128]. Using Gaia's precise astrometry and photometry [44] revealed that some of those clusters are only asterisms and confirmed about 1200 clusters. Looking at groups in a five-dimensional space (positions, proper motions and parallax), [45, $52,53,213$ ] have discovered hundreds of clusters undetected until now to limiting magnitudes of $G \sim 17-18$. Future data releases will extend this limit to $\sim 5 \mathrm{kpc}$, potentially increasing the number of mapped clusters to $\sim 10 \%$ of the Milky Way total.

A mission with 14-20 times better proper motion accuracy for common stars and NIR capabilities would cover half of the Galaxy or more, including regions towards the Galactic Centre. It would thus enable us to probe a much more diverse range of environments for cluster formation, in terms of stellar and gas density, and metallicity (all of which increase towards central regions). Cluster properties such as stellar density (cluster size and number of members), dynamics, and age as a function of location within the Galaxy will provide strong constraints on models of star formation. Furthermore, the range of stellar types will be increased to include low-mass stars (subsolar) whose fluxes peak in the NIR. This will allow a better characterisation of the IMF at low masses and its dependence on the environment, and will again provide clues on the physics of star-forming processes. With an increased astrometric accuracy it will be possible to access the more crowded inner regions of each cluster.

The internal dynamics of embedded clusters are needed to study the small scale structure of the molecular clouds and shed light on the conditions and physics of the cluster at the epoch of star formation. Likewise the bulk dynamics of these clusters allow one to probe the large scale structure of the molecular clouds and the kinematics tell us about the state of the gas at the epoch of formation. A NIR option would allow the dusty star-forming regions to be globally surveyed for the first time. These important stellar birth places are in the regions where extinction intervenes to make visible observations, such as those of Gaia, difficult. A new mission will also help to constrain the distances of any gas clouds whose line-of-sight kinematics are similar to nearby stars while 3-D dust maps of the ISM need good distances.

Open clusters are excellent tracers of the Galactic potential if their tangential motions and accelerations are accurately measured, particularly as the cluster distances can be very accurately constrained from astrometry and photometry. Accurate proper motions from combined missions would allow us to derive cluster membership more clearly. This is already partially possible with the Gaia data alone but a combined mission separated by 20 years would allow internal accelerations and the initial mass functions to be studied and allow us to probe much larger volumes of the Galaxy. 
ALMA, ALMA-VLBI, and the SKA will help us to understand the way gas clouds evolve (condense, fragment) and form stars with unprecedented detail. However, it will not be possible to perform astrometry on a large scale with these instruments. A new NIR astrometry mission is an excellent instrument to complement ALMA, as it will enable us to reveal the position-velocity phase space of low mass, newly born stars, etc. A new mission will be a unique opportunity to compare the velocities of these new stars to the dynamics of the gas, thus fully understanding the star-formation processes.

\subsection{Single and multiple stars}

High-precision astrometry in the NIR will have an impact on a wide variety of stellar physics topics. With NIR wavelengths we will be able to reach regions in the Galactic plane and near the centre of the Galaxy which are particularly interesting for studies of star formation, as they are the common birthplaces of stellar clusters and associations. For binary stars and multiple systems the measurements of astrometric orbits and distances will make it possible to determine accurate masses of the components of binary systems, which are crucial to advancement in several areas of stellar physics. For a planetary system, the mass of the host star must be known in order to determine the masses of the planets. Masses of single stars are determined with the help of stellar evolution models, and have uncertainties of up to $30 \%$, leading to $15 \%$ uncertainty in planet masses (see e.g. the exoplanets.org database). This is particularly true for low-mass stars, which also seem to be the most-frequent planet hosts [72]. Masses of binary stars determined from astrometric orbits together with observables to be compared with predictions from stellar models provide the necessary constraints for developing the most realistic models.

More exotic objects, which will benefit from improved mass estimates from binary orbits, are neutron stars and stellar-mass black holes. This science case is similar to those envisaged for the SIM astrometry mission (see [237], and references therein). The equation of state for matter at densities beyond those of nuclei is not known. Several proposals exist, with different predictions for neutron star masses, and current mass estimates are compatible with all of those. Likewise, large uncertainties are associated with current mass estimates for Galactic black holes and hamper our understanding of their nature. In addition, dynamics of neutron star or black hole binary systems measured from accurate proper motions will allow to constrain their formation mechanism.

Simulations indicate that, with upcoming data releases, Gaia will vastly improve the detection and characterization of exoplanets, brown dwarfs, white dwarfs, neutron stars, and black holes as hidden companions to more luminous, non-degenerate stars [11]. These populations will substantially impact a broad range of fields. For instance, the astrometric detection of even a handful of stellar-mass black holes with main sequence or giant companions will resolve outstanding problems in accretion physics and black hole formation physics [38]. A NIR mission would allow exoplanet or compact object detection around cooler, lower mass stars than those observed by Gaia. The astrometric signal for a body of mass $M_{b}$ orbiting a star of mass $M_{*}$ goes as $M_{b}\left(M_{b}+M_{*}\right)^{-1}$, therefore astrometric detection becomes easier for lower-mass stars 
at a given distance. In practice this means that the "distance horizon" for detection is increased (resulting in expanded samples) and systems can be detected to smaller masses (e.g., Neptune-like planets). A similar limit will hold for long-period binary stars: if the period is 30 years and both have masses $0.5 M_{\odot}$, the semi-major axis will be 12 AU (slightly larger than Saturn's orbit). Since longer-period binaries have larger astrometric signals, these binaries will be detectable out to larger distances (Andrews et al., in prep).

Proper motions derived from short missions can be affected by systematic errors due to the motions in unresolved binaries and the error depends on the mass and orbital period of the pair. For instance, unresolved double stars can be detected due to the large residuals in the astrometric solution, which is normally based on linear motion for a single star [76]. Additionally, a comparison of the proper motions from each mission with a joint solution from both missions can improve binary population statistics and reveal the acceleration in the orbit. More detailed non-linear modelling for multi-star systems is then needed for the classification of such binaries and this is greatly enhanced by a new mission and the longer time baseline leading to new discoveries.

At the same time, the precise astrometry from Gaia affords the identification of wide binaries, which are detected as resolved stars with consistent proper motions and parallaxes $[8,177,178]$. Since the stars in such binaries were formed from the same pre-stellar material, such binaries can be used to test the chemical consistency of stars from the same population, and can therefore be used to calibrate chemical tagging as a method of uniquely identifying stellar sub-populations in the Milky Way [9, 10, 215]. With precise enough astrometry, these binaries can be used to test gravity in the low-acceleration regime [18, 183]. However, the tightest constraints are placed on binaries with orbital separations in excess of $0.1 \mathrm{pc}$. At these separations astrometric measurements from Gaia are not yet precise enough to discern between various gravity laws [184]. With a factor of 14-20 improvement in proper motion measurements resulting from the addition of a NIR mission, the combined Gaia and GaiaNIR data set would provide direct, precise measurements of gravity in the low-acceleration regime.

\subsection{Brown dwarfs}

A strong limitation of Gaia is that it observes in the visible. As such, it is not sensitive to very red objects and sources in high-extinction regions. In spite of it, Gaia has been able to identify over 2000 ultracool dwarfs, a diverse ensemble of sources that include very low-mass stars and brown dwarfs in the solar neighbourhood with spectral type later than M7 and that emit the bulk of their radiation in the $J H K$ bands [216]. However, of them only a few hundred have L spectral type $\left(T_{\text {eff }} \sim 2200-1300 \mathrm{~K}\right)$, less than ten have T spectral type ( $\left.T_{\text {eff }} \sim 1300-550 \mathrm{~K}\right)$, and none have Y spectral type $\left(T_{\text {eff }} \lesssim 550 \mathrm{~K}\right)$. Besides, most early-L dwarfs in the solar neighbourhood are mainsequence stars with masses above the hydrogen-burning limit [129]. As a result, only a few dozen nearby brown dwarfs have actually been detected by Gaia [217]. 
A comparable number of more distant, younger brown dwarfs, with mid-M spectral type but over-luminous, have also been detected by Gaia in open clusters and associations of 3-10 Myr, at $150-400 \mathrm{pc}$, and of low extinction (especially $\sigma$ Orionis and Upper Scorpius). A new NIR astrometry mission would provide us with homogeneous, accurate, parallactic distances of a very large sample of both ultracool dwarfs in the solar neighbourhood and brown dwarfs in a larger number of young starforming regions and juvenile open clusters (e.g. $\rho$ Ophiuchi, Orion Nebula Cluster, Pleiades) down to, perhaps, the deuterium-burning mass limit [43]. Such an unbiased sample of very low-mass stars and substellar objects with masses down to the planetary regime and with very different ages will offer a magnificent panorama of the bottom of the initial mass function, which is a topic in astronomy that triggers vigorous discussion (see for example [20]).

\subsection{White dwarfs}

White dwarfs (WD) are the final evolutionary stage of intermediate and low mass stars (about $95 \%$ of all stars end as WD remnants). Their study provides key information about the late stages of the star's life, and also of the structure and evolution of the Galaxy because they have an imprinted memory of its history [114, 143]. The LISA mission will study the Milky Way's structure from the gravitational waves produced by close double WDs throughout the whole Galaxy, and in combination with their motion information obtained from electromagnetic observations the Milky Way's disc and bulge masses can be derived [137]. Through comparison of the empirical and theoretical Luminosity Functions (LF) of WDs one can derive the age of the Galaxy and its star formation rate (see [91] for a review). The LF allows the reconstruction of the IMF.

The Gaia mission has allowed the construction of the largest sample of WDs so far [92], and the first almost complete volume-limited sample up to $100 \mathrm{pc}$ [119]. The analysis of their position in the Hertzsprung-Russell diagram has shown a bifurcation not predicted by the models, which cannot be entirely attributed to the existence of a population of helium-rich atmosphere WDs [119]. In addition, the double WD binary population detected by Gaia is of particular interest to the LISA community, although many more double WD binaries are hidden in the dusty Galactic plane making their multi-messenger electromagnetic characterization more difficult.

In this context, a new NIR astrometry mission will be extremely important. As an example, pure-H and pure-He atmospheres can only be distinguished in the NIR regime [49]. Especially important for the construction of the empirical LF are the oldest members of the WD populations of thin and thick discs and halo, which were formed by high-mass progenitors and evolved very quickly to the WD stage, being very cool and faint in the present day. A NIR facility will allow the characterization of the cool WD population much better than Gaia in the optical, which has limited capabilities [49] both in terms of detection and parametrization. Furthermore, a new mission will be able to detect and characterise close WD binaries in the dusty regions of the Milky Way's disc and bulge necessary for LISA studies Fig. 2. 

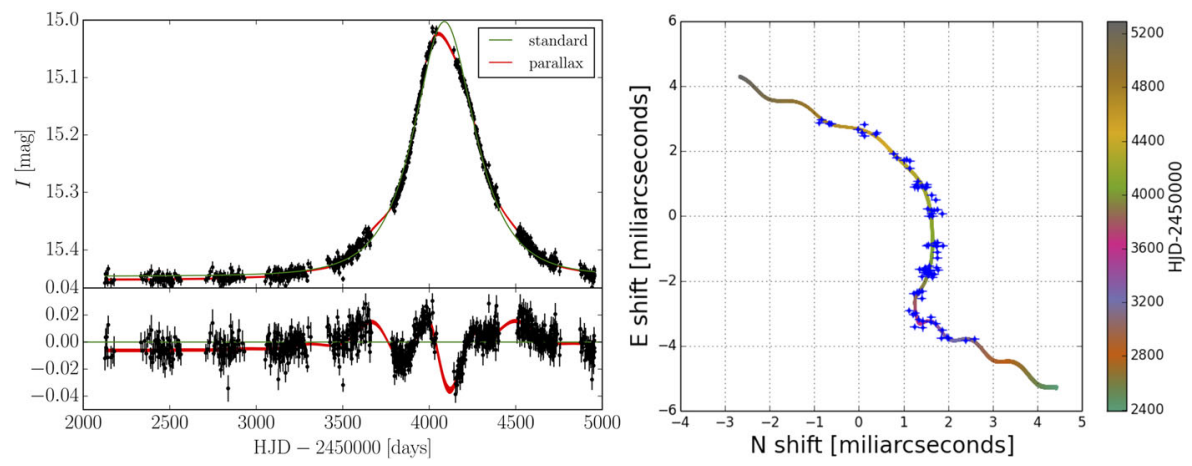

Fig. 2 Microlensing event, OGLE3-ULENS-PAR-02, a candidate for a $\sim 10 \mathrm{M}_{\odot}$ single black hole. Left: photometric data from OGLE-III survey from 2001-2008. Right: simulated position of the source (and the centre of light of the two microlensing images) for a similar event, combining source's proper motion, parallax and astrometric microlensing signal. Blue data points are simulated GaiaNIR observations

\subsection{Astrometric microlensing}

Black holes, neutron stars, and brown dwarfs are expected to fill our Galaxy in large numbers, however, such dark objects are very hard to find, as most of them do not emit any detectable light [248]. About thirty years ago Bohdan Paczyński [179] proposed a new method of finding those compact dark objects via photometric gravitational microlensing. This technique relies on continuous monitoring of millions of stars in order to spot their temporal brightening due to space-time curvature caused by the presence and motion of a dark massive object. Microlensing exhibits itself also in astrometry, since the centre of light of both unresolved images (separated roughly by two times the size of the Einstein Ring, $\sim 1$ mas) changes its position while the relative brightness of the images changes over the course of the event (e.g. [70]). Astrometric time-series at sub-mas precision over a range of timescales (depending on the mass and distance of the lens) of several months to years would provide measurement of the size of the Einstein Ring that, combined with photometric light curves, would directly yield the lens's distance and mass. So far (in 2020) there have been thousands of microlensing events discovered in photometry (e.g. OGLE, MOA, Gaia Alerts), however, the mass of the lens has only been measured in a handful of them (e.g. [249]).

At the typical brightness of the microlensing events at $I=19-20$ mag a new NIR astrometry mission would be capable of providing a good-enough astrometric signal for the events discovered in photometry by future Bulge or all-sky surveys (including GaiaNIR itself). The superb astrometric time series combined with microlensing parallax measurements obtained for events lasting more than $\sim 4$ months, will allow the mass and distance of the lens to be measured and the nature of the lens to be derived. A NIR capability would be best for Bulge and Galactic plane microlensing due to lower extinction and more sources to monitor (see for example [126]). 
Detection of isolated black holes and a complete census of masses of stellar remnants will for the first time allow for a robust verification of theoretical predictions of stellar evolution. Discovery of a large number of stellar-mass black holes in the Milky Way could also help constrain the content of dark matter in the form of primordial black holes (e.g., [48]). Additionally, it would yield a mass distribution of lensing stars as well as hosts of planets detected via microlensing.

\subsection{Stellar variability}

Visible-NIR astrometry will enable extensive local tests of stellar standard candles (e.g. [237]). Accurate distances of Cepheids and RR Lyrae stars throughout the Galactic plane, including the Galactic bulge, will result in improved periodluminosity relations, since the key uncertainties of variable extinction and metallicity are significantly reduced in the NIR (see Figure 15 in [7]). By extending these measurements to local group galaxies the validity of these relations can be tested for different chemical environments and galaxy types. For Cepheids, a better understanding of the physics of pulsation may improve the applicability of the period-luminosity relations for determining accurate galaxy distances. Advancement in pulsation models requires accurate masses of Cepheids, which can be obtained from astrometric orbits of binary systems with Cepheid components. [247] have shown that the Cepheids are very difficult to observe towards the Galactic Centre (see their Fig. 4) and at low latitudes (see their Fig. 5) and a NIR-capable mission would help greatly to uncover this difficult region. The Cepheids are also crucial for determining the distance scale which is essential for dark energy studies. The distance scale will be anchored on Gaia parallaxes soon, but the key uncertainty remains: extinction. NIR photometry will help to resolve this problem and significantly contribute to a highly accurate measurement of the Hubble constant, a fundamental quantity for cosmology [201].

Gaia has detected more than 150000 Long Period Variables (LPVs), mainly Mira and Semi Regular (SR) variable stars [172]. LPVs are long period pulsating stars in the final stage of their evolution, on the asymptotic giant branch (AGB). Similarly to Cepheids or RR Lyrae, LPV variables show defined period-luminosity relations making them important stellar standard candles [80]. LPVs also cover a wide range of age, and there is a potential age-period relation [99]. Hence, they can trace the kinematics of different age populations. Moreover, they are much more numerous and brighter than Cepheids, so they could really help in the study of the Galactic structure and extragalactic studies.

The pulsation is accompanied by heavy mass loss which forms a circumstellar envelope of gas and dust which obscures the central star, absorbing its light and re-emiting it in the IR. The more obscure AGBs (the so-called OH/IR stars), those optically invisible, are even brighter than Miras and could be even better distance indicators. However, their period-luminosity relation is barely known due to the dearth of well determined periods [29, 118]. Only a NIR mission would be able to really characterize these stars and to ultimately define their period-luminosity relation. With 
such a new mission it would be also possible to study the effect of the different metallicities in the bulge and the disc on the period-luminosity relation [117], so they could be used as standard candles improving the distance scale.

\subsection{Exoplanetary science}

Around the 2030's space-borne and ground-based exoplanet projects will focus on the characterization of structural and atmospheric properties of exoplanet systems, particularly those hosting temperate, rocky terrestrial planets amenable to the detection of biosignature gases indicating the presence of life. However, there will still be uncharted regions of the parameter space bracketed by mass, orbital separation, and properties of the host stars that can provide critically important constraints on our understanding of all possible outcomes of planet formation and evolution processes. Precision astrometry is particularly sensitive to orbiting planets at increasing separation from the host star, and the measurement of orbital motion in the plane of the sky allows the derivation of the full set of orbital parameters, including the inclination, even for multiple-planet systems (see [221, 224] and, for a real application, [155]). However, in spite of its complementarity with other techniques, the applications of precision astrometry to exoplanetary science have thus far been limited to the characterization of small numbers of known Doppler-detected systems. The required astrometric precision is challenging and it is only with Gaia that astrometric detection and orbital element fitting for large samples of extrasolar planets are about to become routine [223]. Gaia's large compilation of new, high-accuracy astrometric orbits of intermediate-separation (0.5-4 AU) giant planets, unbiased across all spectral types, chemical composition, and age of the primaries, will allow astrometry to crucially contribute to many aspects of the formation, physical and dynamical evolution of planetary systems, particularly given its synergy potential with ongoing and planned exoplanet detection and (atmospheric) characterization programs [223]. However, even after Gaia's ultimate exoplanet catalog publication, key uncharted territories will remain to be explored in the realm of extrasolar planets.

A new NIR astrometry mission will revolutionize the field of exoplanetary science in two ways. First, it will establish the presence of planetary-mass companions orbiting classes of stellar and sub-stellar primaries that cannot be observed with sufficient sensitivity using other techniques and that appear bright in the NIR, enabling a jump of typically a factor of several in achievable astrometric precision with respect to Gaia. These include two particularly relevant samples: 1) hundreds of ultra-cool early-L through mid-T dwarfs in the Sun's backyard ( $d \lesssim 40$ pc, see e.g. [130]), around which GaiaNIR could complete the census of any existing population of cold giant planets out to $\sim 4-5 \mathrm{AU}$ (e.g., [222]), with the possibility to access the regime of cold Neptunes and Super Earths orbiting the nearest, brightest L dwarfs; 2) a statistical sample of maybe thousands of heavily reddened young stars all the way to the bottom of the main sequence in the nearest star-forming regions $(t \sim 3-$ $10 \mathrm{Myr}, d \lesssim 200-300 \mathrm{pc}$, see Section 3.10), around which only GaiaNIR might be sensitive to intermediate-separation gas giants. These unique contributions of a new NIR astrometry mission would provide a) first-time measurements of the efficiency 
of planet formation around brown dwarfs, which is today a matter of hot speculation (e.g., [199]), with very little observational support [102], and b) much improved constraints on the true planetary companion mass function at all separations and at very young ages (when orbital evolution might still be ongoing), in synergy with present and planned high-contrast imaging programs. All the above results will also greatly benefit from improved parallax estimates for the host stars, particularly in the low-mass star and ultra-cool dwarf regime (see Sections 3.10 and 3.9).

Second, systematic GaiaNIR observations of all stellar samples (possibly $\geq 10^{6}$ stars) around which Gaia (particularly a 10-yr extended mission) is sensitive to planetary companions [223] would also bring novel insight on the global architectures of planetary systems. The loss in sensitivity to orbit reconstruction with Gaia beyond the mission lifetime is well known [51, 180, 195, 225]. A new mission launching approximately in, for example, 2040 and using a similar scanning law to Gaia's would allow accessing gas giant companions with orbital periods in the $\sim 30-35$ year interval, depending on the new mission's lifetime. Astrometric detection of a planet with a non-zero eccentricity can be done with a small fraction of the orbit, so we may be able to determine much longer period planets also. Tests show that the presence of a gap in the data collection does not introduce too large biases in the recovered orbital elements. Aliases have been found to be easily identifiable, with their presence greatly mitigated when at least one mission is extended. With two short missions $(5+5$ year) we expect fractions of false positive and false negative detections under $\sim 10 \%$; and significantly less for two long missions $(10+10$ year). The Gaia+GaiaNIR unbiased census of bright stars screened for gas giants at Saturn-like distances and the possibility of determining the frequency of multiple systems containing both giant planets at Jupiter- and Saturn-like separations would allow, for instance, identification of exact Solar System-like architectures, complementing at intermediate separations the systematic searches for temperate terrestrial planets around the nearest solar-type stars with extreme-precision radial velocities (e.g., [81]), and the characterization of potentially habitable rocky planets transiting Sun-like stars identified by the PLATO mission [196].

\section{Improved proper motion science cases}

A portion of the rich and violent history of the Milky Way has become clear due to Gaia data. The Milky Way underwent a major merger with another galaxy in its early life (around $10 \mathrm{Gyr}$ ago), and the remnant of this merging galaxy makes up the majority of the Milky Way's inner stellar halo [23, 105]. Gaia data have also been used to find the remnants of another significant merger in the early life of the Milky Way [173], and to detect and characterise nearby streams of stars, formed when satellite galaxies or globular clusters are pulled apart in the potential of the Milky Way $[152,189]$. However it will not be sufficient to discover and characterise most of the stream-like structures in the halo. Improvements in the accuracy of proper motions would allow a new mission to resolve tangential motions in streams and local dwarf galaxies, with a potential accuracy of $2-3 \mathrm{~km} \mathrm{~s}^{-1}$ for specific samples 
out to $\sim 100 \mathrm{kpc}$. Additionally, improved proper motions will also be crucial to help disentangle the mixed populations in the bulge region. This is only possible by exploiting the long time baseline allowed by combining Gaia measurements with those from a future astrometric mission.

Furthermore, using the Gaia proper motions of tracers in the Milky Way halo, the potential of the Milky Way, and therefore its mass, was measured out to around $20 \mathrm{kpc}$ [188, 244, 246], but this is only thought to be 10-20 percent of its total mass. The total mass of the Milky Way is an important quantity because it tells us where the Milky Way fits in a cosmological context. The local group has been called the "Ultimate Deep Field" [36] because it probes a large co-moving volume at high redshift, and can be observed with great detail. Important questions in cosmology such as the "Missing satellite" problem and the "Too Big to Fail" problem are related to whether the Milky Way is typical of galaxies seen in cosmological simulations. To make this comparison in a fair way one needs to know the mass of the Milky Way.

\subsection{The halo and streams}

Streams in the Milky Way halo are formed when satellite galaxies or globular clusters are pulled apart in the tidal potential of the Milky Way. The stars then drift apart because they are on different orbits and form a (typically thin) band of stars across the sky. This makes them sensitive probes of the Milky Way's potential (e.g. [33, 151]) because the model of their formation is relatively straightforward, and their narrow distribution across the sky provides tight constraints in some ways (though ones that require more phase-space information if they are to be useful). This will allow us to determine the dark matter distribution at large radii, including any flattening of the potential, and the total mass of the Galaxy. A future astrometry mission would provide highly accurate proper motions and more accurate distances to these stars, which will allow much more precise determination of the potential of the Milky Way at these large radii.

A further exciting opportunity opened up by improving proper motion measurements for stars in Milky Way streams is that by finding "gaps" in the streams (more easily found if we have proper motions as well as positions for stars), we might see the influence of dark matter sub-haloes in the Milky Way's halo even though they contain no stellar matter. It is hypothesised that such sub-haloes are common in the Milky Way halo, but the only way to detect them is through their gravitational interaction with luminous matter. When one of these dark matter haloes has a flyby encounter with a stellar stream, it can create a disturbance (or gap) that may be detectable with sufficiently accurate proper motion measurements [32, 46, 206]. The dynamically cold nature of the stellar streams makes them ideal candidates for detecting these flybys.

Another method of determining the mass of the Milky Way out to large radii is by modelling the motion of a tracer population in the outer halo that can be assumed to be in equilibrium. The major difficulty with this endeavour has historically been that there is a degeneracy between the mass enclosed within a given radius and the orbital anisotropy of the tracer population [64]. This is only a problem because the only available measured velocity component was the line-of-sight velocity, and for 
stars in the outer halo this component is nearly identical to the Galactocentric radial velocity. The availability of accurate proper motions for these stars will break this degeneracy and enable us to learn the mass of the Milky Way from the tracers we see.

Further, it is an open question what fraction of the stellar halo is in substructures and what fraction is in a smooth component, and how much impact the LMC made [75]. Sometimes substructure can be found as an over-density on the sky, but the availability of accurate proper motions will make it easier to find substructure in the larger phase-space and even the dark matter wake due to the LMC $[24,90]$.

All of the above questions cannot be answered by Gaia alone due to the low accuracy of the proper motions at great distances $(\sim 10 \mathrm{kpc})$. However, a new mission combined with the older Gaia/Hipparcos/Tycho-2 catalogues would give the much longer baseline needed to get very accurate proper motions; remember a factor of 14-20 better in the two proper motion components (see Section 2.1) to open up these critical areas to understand the Galaxy in a much greater volume than is currently possible.

\subsection{Hyper-velocity stars}

Brown [39] and references therein, have shown that the origin of hyper-velocity stars (HVSs) is most likely due to gravitational interactions with massive black holes due to their extreme velocities. Future and very accurate proper motion measurements are a key tool to study these objects. Precise proper motion measurements, due to the largely radial trajectories of these stars, combined with radial velocities can provide the three dimensional space velocity of these objects. Unfortunately, known HVSs are distant and on largely radial trajectories [39]. Some HVSs originate in the Galactic Centre while others have an origin in the disc but an origin in the Magellanic Clouds or beyond is also possible. Proper motion accuracies from Gaia (or combined missions) are needed to reconstruct their trajectories and distinguish between the different possible origins.

Gnedin et al. [96] showed that precise proper motion measurements of HVSs give significant constraints on the structure (axis ratios and orientation of triaxial models) of the Galactic halo. Triaxiality of dark matter halos is predicted by cold dark matter models of galaxy formation and may be used to probe the nature of dark matter. Using data from Gaia and a new NIR mission, combined with distances and radial velocity measurements, would allow for a factor of 14-20 improvement in the accuracy of the proper motions to advance our understanding of HVSs and the Galactic potential. Adding the NIR capability would also allow us to probe more deeply into the Galactic Centre and potentially detect small populations of HVSs closer to their ejection location.

\subsection{Co-moving stars}

Simulations [121] have demonstrated that co-moving stars, even those that are widely separated $(\sim 10 \mathrm{pc})$, are mostly co-natal. This picture is further borne out by observations [9, 215], supporting the idea co-moving stars are "clusters of two." Such objects are of high interest in Galactic archaeology because they are significantly 
more abundant than their open cluster counterparts. The lack of nearby open clusters for detailed spectroscopic follow-up has always been a significant roadblock to calibrating stellar models. The vastly abundant co-moving (and co-natal) stars have enormous potential in changing the landscape of stellar model calibrations (e.g., spectral models, isochrones). The frequency of co-moving stars is a tell-tale sign of Galactic perturbations (e.g., giant molecular clouds and dark matter substructures) and clustered star formation. Most co-moving stars were formed in a clustered star formation environment and were subsequently disrupted by Galactic perturbations [122]. It is the competition of these two effects which sets the number density of co-moving stars. Modelling the statistics of co-moving stars will thus put stringent constraints on these two subtle properties that are otherwise not directly detectable. A new mission will be an important step forward in finding more of these co-moving pairs.

\subsection{Local group}

Astrometrically resolving internal dynamics of nearby galaxies, such as M31, dwarf spheroidal galaxies, globular clusters, the Large and Small Magellanic Clouds (LMC, SMC), sets requirements on the accuracy. For example, the LMC has a parallax of $20 \mu$ as and an accuracy of about $10 \%$ is needed, which is just within the reach of Gaia. Precise mapping of dark matter (sub-)structure in the local group and beyond is possible with accurate proper motions. Gaia can only just directly measure internal motions of nearby galaxies. Combining proper motions from two Gaia-like missions opens up the tantalising possibility of accurately measuring their internal motions and thus resolving the dynamics within the Local Group. A number of science cases are within reach:

- Using photometric distances to the LMC allows us to learn about internal dynamics and structure.

- Using proper motions to determine rotational parallaxes to M31, M33 and other local galaxies.

- Dynamical measurements of the mass distribution of the Milky Way and M31.

- Probe the internal kinematics classical dwarf spheroidal galaxies.

- Mapping the dark matter sub-structure throughout the local group out to M31.

- Resolve the core/cusp debate ${ }^{4}$ with 6D phase space information.

- Ultra-faint satellite galaxies would be accessible and their orbits could be better determined than by Gaia alone [214].

Dwarf spheroidal galaxies (dSph) are fascinating systems and likely fossils of the re-ionisation epoch ${ }^{5}$ In order to study their internal proper motions there is a need to disentangle the Milky Way environment using very accurate proper motion measurements. The dSphs are small, almost spherical, agglomerates of stars that orbit more

\footnotetext{
${ }^{4}$ Discrepancy between observed dark matter density of low-mass galaxies and density profiles predicted by cosmological N-body simulations.

${ }^{5}$ This paragraph is extracted from an unpublished note from Rodrigo Ibata.
} 
massive hosts. In the local group these $\mathrm{dSph}$ galaxies are the most numerous class of galaxies, with 15 discovered around the Milky Way, 25 around the Andromeda galaxy, and possibly one orbiting M33. Several other nearby galaxies have also been found to harbour these satellites. It is believed that hundreds of dSphs and globular clusters were accreted during the formation of the Galaxy, and their remnants should be visible as coherent phase-space structures. The challenge is of course to identify the stars with common dynamical properties.

An interesting aspect of these galaxies is their identification as possible cosmological "building blocks" of the type predicted by cold dark matter theory. A generic feature of a Universe in which the dominant form of matter is in the form of cold dark matter is that galaxies should be surrounded by tens of thousands of dark matter satellites with masses comparable, or larger, than those of dwarf galaxies. While the number of observed satellites is very much lower than this, it has often been suggested (or assumed) that baryons collapsed to form substantial stellar populations in only the most massive of the dark satellites. Other dark satellites may have lost their baryons, or had star-formation suppressed for a variety of reasons. Thus the dwarf satellite galaxies may represent the surviving remnants of the original population of primordial galaxies that coalesced and merged to form our Milky Way.

To confirm this picture we need to understand the internal dynamics of dwarf galaxies, and to date, the necessary observations to test these ideas have remained beyond our reach. The issue is that there is a trade-off between dark matter content and tangential anisotropy and/or tidal heating. A system with stars on preferentially tangential orbits to the Galaxy and that has also been heated by repeated tidal encounters with the Milky Way can masquerade as a highly dark matter dominated galaxy. The only way to be sure of the true dark matter content is to measure the internal proper motions of the stars in the dwarf galaxy, not only the bulk proper motion of the system (feasible with Gaia). Only the combination of Gaia and GaiaNIR can hope to achieve this challenging goal.

\subsection{Prospects for exoplanets and long period binaries}

Proper motions from short missions are affected by systematic errors due to the motions in unresolved binaries and the error depends on the mass and orbital period of the pair. Such systems are poorly surveyed at present. The unresolved double stars can be detected due to the large residuals in the astrometric solution, which is normally based on linear motion for a single star. Additionally, a comparison of the proper motions from each mission with a joint solution from both can improve binary population statistics and reveal the acceleration in the orbit. More detailed non-linear modelling for multi-star systems is then needed for the classification of such binaries which is greatly enhanced a new astrometry mission and the longer time baseline, leading to new discoveries.

Kervella et al. [127] and Brandt [37] have shown that Hipparcos and Gaia astrometry can be used to measure changes in proper motion, i.e., accelerations in the plane of the sky, which opens the possibility to identify long period orbital companions otherwise inaccessible while also constraining their orbits and masses. Synergies between the two Gaia-like missions and the PLATO mission [196] would allow 
a comprehensive comparison of the short period planets detected by PLATO and the long period planets from two Gaia-like missions giving quantitative insight into possible Solar System analogues.

\subsection{The solar system}

A strong impact of Gaia and of the proposed follow-up mission GaiaNIR is on the study of the population of small Solar System bodies, asteroids, comets, and planetary satellites. Precise fundamental ephemerides of the planets in the Solar System rely mostly on radar and spacecraft data for the inner Solar System, spacecraft data for Jupiter and Saturn, and lunar laser ranging for the Moon. The link to the ICRF is provided by VLBI observations of the spacecraft $[82,111]$. Radar techniques have also been exploited on small bodies, such as Earth-crossing asteroids (NEOs) but remote optical observations are still required, as only they are capable of covering the largest sample of object sizes and distances.

Infrequent spacecraft flybys of asteroids are very useful for determining masses and other physical properties, but do not always constrain the orbital parameters very well. So far only 20 small bodies have been visited, while a NIR survey will reach hundreds of thousands. For the distant outer planets, the modern astrometric technique is to observe the positions of the natural satellites and infer the position of the centre-of-mass of the planet-satellite system. Better orbits for the satellites are also of interest for modelling the dynamical history of the satellite systems. In the inner Solar System, there is much current interest in near-Earth asteroids (NEOs) that pose some risk of colliding with Earth in the future. These are generally small, faint objects that do not have long-term (or any) observing histories, as their orbits are closer to the Sun than the Earth's orbit making their detection particularly challenging. Estimating the future paths of these objects and their risk to Earth therefore requires accurate absolute astrometry. Also, to trace back their origin to the Main Belt, the history of collisional families must be studied. Their spread in the orbital element space can be used as an astronomical clock for their evolution [227, 243], and is driven by the Yarkovsky acceleration (recoil force due to the emission of thermal photons [65]). Measuring the Yarkovsky effect, which causes a secular drift in the orbital semi-major axis, is one of the main challenges of asteroid science.

Today, Gaia is demonstrating the potential of high accuracy astrometry and photometry in the dynamical and physical characterisation of the population of small Solar System bodies. Among modern challenges that require accurate astrometric measurements and precise orbits are, determining the masses of asteroids, predicting stellar occultations by asteroids [230], monitoring the impact risk of potentially hazardous near-Earth objects, which may exhibit very fast apparent motion, and, conversely, the determination of the orbits of very slow and faint bodies, the so-called Trans-Neptunian Objects (TNOs).

Asteroid masses can be determined by very precise astrometry before and after mutual encounters [106]. However, asteroid-asteroid encounters that are useful in determining asteroid masses are infrequent events for ground-based observers, due to the limitations in the astrometric accuracy. Unknown masses of asteroids contribute "noise" in the ephemeris of the planets in the inner Solar System [228], although the 
last decade's worth of ranging data from Mars spacecraft have allowed for improvements in the determination of the masses of the asteroids that significantly perturb that planet's orbit [141]. However, Gaia astrometry is accurate enough to directly provide masses of the largest 100 perturbers [171]. Gaia astrometry of stars is also being effectively used to improve stellar occultation predictions. For instance, the use of Gaia astrometry in the case of the occultation targets by the TNO 2014 MU69 has permitted positive observations and provided strict constraints on its orbit. This success has been fundamental for planning of the flyby by the New Horizons (NASA) space probe [42].

While successful occultations and mass determination are based on the instantaneous knowledge of very accurate orbits, taking into account subtle long-period, or secular effects, requires observations over a long time span. For instance, measuring the Yarkovsky acceleration directly in the Main Belt would give a major contribution to our understanding of asteroid families and to the mechanisms that lead to the injection of asteroids on Earth-crossing trajectories. The best current Yarkovsky determinations are based on the joint use of both Gaia and previous ground-based astrometry spanning several years (or decades). They can be successful only when the orbit uncertainty (on the semi-major axis) reaches a few $\sim 100 \mathrm{~m}$ or less. The low accuracy of pre-Gaia asteroid astrometry is a clear limitation, as the average RMS of the residuals for CCD astrometry in the Minor Planet Center database is 400 mas [68]. Careful data selection, and zonal de-biasing strategies for old positions derived relatively to stars from pre-Gaia catalogues, are required [47, 55, 79]. With this approach, Gaia is expanding the limit of detection for Yarkovsky from the NEO region to the inner Main Belt.

The possibility of a direct, accurate measurement of Yarkovsky deep into the Main Belt on collisional family members, would be a major breakthrough brought about by an astrometric follow-up mission. The expected improvement factor in the orbit accuracy obtained by combining the data of two astrometric missions on a 20 year baseline, is similar to the improvement estimated for the stellar proper motion, in the case of NEOs, Main Belt asteroids, or natural planetary satellites. For them, observations will be distributed all along their orbits. On the other hand, for small bodies whose orbital period is substantially longer than the Gaia mission alone the gain in accuracy is potentially much higher, as in this case it depends strongly on the fraction of the orbital period that is covered. This is the case for objects exterior to the orbit of Jupiter such as the Centaurs (periods between 20 and 150 years) or the TNOs. This population also harbours the highest priority target for stellar occultations, the only technique capable of providing accurate size measurements and vertical density profiles of their atmospheres (such as for Pluto) at those distances.

The second major contribution of a NIR mission would be its complementarity with respect to the spectral coverage of Gaia. In fact, Gaia is building a new taxonomy based on the visible, but asteroid classifications including the NIR breaks the degeneracy of certain spectral types and bring additional information on the composition [67]. For wavelengths exceeding 1 micron, slope changes can occur and wide absorption bands, diagnostics of the mineralogy, can affect the slope at the extreme of the GaiaNIR spectral coverage (1.8-2 microns). Being able to retrieve mineralogy information for hundreds of thousands of objects would be a major milestone with a 
direct impact on the models describing the formation and the evolution of the Solar System [66]. GaiaNIR would then be the largest NIR-inclusive survey of the asteroid population, providing a new portrait of the asteroid belt composition. This constitutes a clear advantage, as physical properties such as surface composition, or rock porosity, directly affect the Yarkovsky effect and are related to the properties of the family parent bodies. Extension in time and wavelength coverage are thus complementary aspects that give a clear strength to GaiaNIR in Solar System science.

\subsection{Astrometric radial velocities}

High-accuracy astrometry permits the determination of not only stellar tangential motion, but also the component along the line-of-sight. Such non-spectroscopic (i.e. astrometric) radial velocities are independent of stellar atmospheric dynamics, spectral complexity and variability, as well as of gravitational redshift. For a detailed discussion see [71]. There are three methods for measuring astrometric stellar radial velocity: a) changing annual parallax, b) changing proper motion (perspective acceleration), and c) changing angular extent of a moving group of stars. All three have significant potential for a combination of Gaia and a new astrometric mission. The baseline design of GaiaNIR does not envisage any spectrograph on-board, but perspective acceleration will allow to measure velocity vectors up to a few ten's of parsecs. This may be relevant for brown dwarfs, for nearby exoplanets ejected from their host star systems and for objects at the outer edges of the Solar System.

\section{Maintenance of the celestial reference frame}

Celestial Reference Frames (CRFs) represent catalogues of sources with accurately known positions (possibly as functions of time). CRFs are important for a number of reasons:

- To refer positions of fixed or moving sources

- To detect tiny motions (e.g. exoplanet detection by relative astrometry)

- To quantify the motion of sources without any kind of bias:

- Motion of stars in the Galaxy

- Differential rotation of the Galaxy

- Dynamics of star clusters

- Rotational and translational motion of external galaxies

- Dynamics of the Solar System

- Source cross-identification in $\gamma$-ray, X-ray, visible, IR and radio wavelengths

- To monitor the rotation of the Earth and study tectonic plate motions using ground-based VLBI

- Angular positions (and distances) of quasars, galaxies, stars, planets, spacecraft navigation, GNSS maintenance, etc.

Gaia is expected to provide the first highly-accurate Celestial Reference Frame (Gaia-CRF) in the optical based on extragalactic sources, thus being less sensitive to 
possible proper motion errors of the sources. The uncertainties of typical individual positions of Gaia-CRF based on 5 years of data is 0.1 mas. This accuracy will degrade over time because of errors in the spin of the Gaia-CRF and in the proper motions due to the acceleration of the Solar System barycentre relative to the rest frame of the quasars. These uncertainties can be expected at the level of about $0.003 \mathrm{mas} / \mathrm{yr}$, so that the uncertainty will double within 50 years after the reference epoch of Gaia.

However, one million quasars expected in the Gaia-CRF represent a tiny fraction of the Gaia sources. With the typical angular distance between the quasars of about 6 arcminutes the Gaia-CRF is not dense enough to provide a suitable reference grid needed for forthcoming Extreme, Giant and Overwhelming telescopes but also for smaller instruments currently operating or being planned. Moreover, most of the quasars of Gaia-CRF are rather faint (between magnitudes $G=19$ and 21) so that the accessibility of the reference frame is given only in the optical and almost exclusively in that interval of magnitudes.

For this reason the whole Gaia catalogue with its accurate proper motions of stars is de facto used as a standard CRF. Because of the errors in proper motions the accuracy of this frame degrades with time. The accuracy degradation is almost linear in time with the slope equal to the error in the proper motions. This is depicted on the left pane of Fig. 3. With proper motions of up to 20 times better than for Gaia, the degradation of the common reference frame becomes much slower (right pane of Fig. 3). Due to the observational principles of Gaia there are six degrees of freedom the orientation $\epsilon=\left(\epsilon_{x}, \epsilon_{y}, \epsilon_{z}\right)$ and the spin $\omega=\left(\omega_{x}, \omega_{y}, \omega_{z}\right)$ of the resulting reference frame. The orientation is determined using a set of a few thousand radio ICRF3/VLBI sources with known positions and proper motions while the spin is determined using a set consisting of hundreds of thousands of quasar-like objects identified in the Gaia catalogue using external photometric surveys of quasars.

The need for a global survey mission like Gaia to maintain the realisation of the $\mathrm{CRF}$ is a science objective in itself as it lies at the heart of fundamental astrometry and provides a reference grid for much of modern observational astronomy. An important aspect of reference frames is to link them, cross-matching with absolute coordinates, to reference frames at other wavelengths to produce reference grids for various surveys. This requires the maintenance of the accuracy of the Gaia visual reference
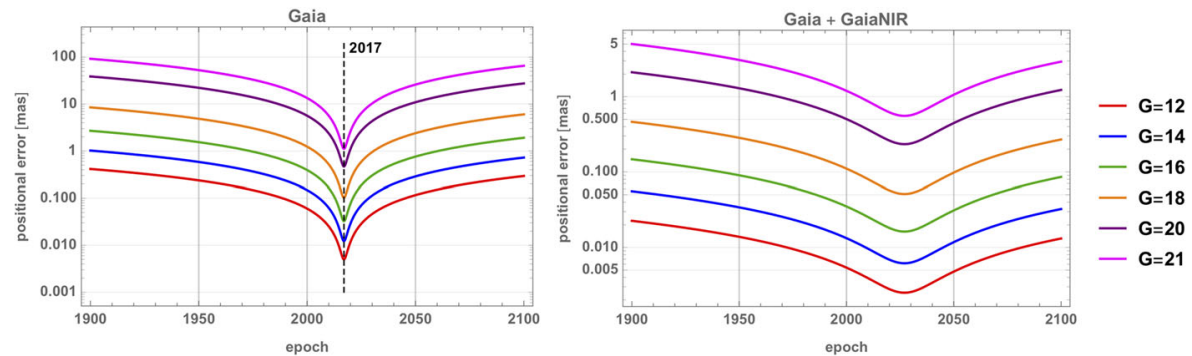

Fig. 3 Degradation of the astrometric accuracy of the individual sources in the Gaia catalogue (left pane) and of the common solution using 10 years of Gaia and 10 years of GaiaNIR data (right pane) depending on the $\mathrm{G}$ magnitude and time from the reference epoch (J2017 for the Gaia catalogue and a mean epoch of Gaia and GaiaNIR taken here as J2027). Note the very different vertical scales 
frame at an appropriate density that is useful for new surveys. A key improvement is to expand the Gaia visual reference to the NIR, increase its density in obscured regions and to then link this new reference frame to the ICRF. Maintaining the visibleNIR reference frame is a vital service to the community and is not feasible from the ground.

Experience in comparing astrometry at different wavelengths has been gained from cross matching the optical Gaia-CRF and the radio ICRF2/ICRF3. The socalled radio-optical offsets have been detected and are being extensively discussed in the literature $[16,150]$. Although these offsets are very important for the discussion of individual objects, they are significant for a small fraction of sources and also uncorrelated from one source to another. This is therefore only an additional source of noise. One can also expect that quasars have astrometric variability at the $10 \mu$ as level due to internal structure and activity.

Eventually, the quasars will be found from the observational data of Gaia itself using quasar classification and this will also hold for future missions. Bailer-Jones and Smith [17] developed a method using Gaia photometry by low-dispersion spectra and showed with simulated data, that it is possible to achieve a pure sample of quasars (upper limit on contamination of 1 in 40000) with a completeness of $65 \%$ at $\mathrm{G}=$ 18.5 , and $50 \%$ at $\mathrm{G}=20.0$, even when quasars have a frequency of only 1 in every 2000 objects.

With a different approach [103] aimed to discover quasars using only their lack of proper motions. The technique has been further validated after the release of the Gaia DR2 data [104]. Astrometric selection of extragalactic point sources also has the potential to select other extragalactic point sources, e.g. potentially new classes of objects.

It is well known that some quasars may have non-zero apparent proper motions and parallaxes (the latter because of time-dependent proper motions projected on the 1-year parallax pattern) due to the quasars internal structure [231], so that only some quasars can be found using purely astrometric approach. Nevertheless, purely astrometric selection is attractive since it is unbiased by assumptions on spectra and photometry. The incompleteness of quasar samples based on selection by visible photometry has been studied intensively for many years and it is now well established that such samples miss a substantial number of, in particular dust-reddened, quasars (see, e.g., [86, 140] for recent studies). This is a serious problem for a number of important issues in astrophysics starting with the study of the quasar phenomena itself. For example, Broad Absorption Line quasars are systematically underrepresented in quasar samples.

The results of [103] show that with the expected accuracy of Gaia astrometric selection gives $20 \%-50 \%$ of stellar contaminations depending on the Galactic latitude. In the set of sources observed by both Gaia and GaiaNIR, due to more accurate proper motions one should expect a truly dramatic improvement: the percentage of stellar contaminants will be reduced by a factor of up to 400, thus leading to an extremely clean quasar sample everywhere on the sky.

In conclusion, a new NIR astrometry mission will be necessary in the coming decades in order to maintain the visible realization of the CRF. This on its own is a strong science case due to the need to maintain dense and accurate reference grids 
for observational astronomy in the visible. However, the addition of NIR astrometry will increase the density of this grid in obscured regions and provide a link to the ICRF in a new wavelength region and drastically improve the quality of the Celestial Reference Frame.

\subsection{Proper motion patterns, real time cosmology and fundamental physics}

A number of specific proper motion pattern of quasars are expected and represent a scientifically exciting topic by themselves. A first sort of systematic pattern in proper motions of quasars comes from the acceleration of the Solar System relative to the rest frame of quasars causing a drift of secular aberration [16, 21, 78, 101, 131, 134, $138,153,220]$. This acceleration is dominated by the acceleration from the motion of the Solar System in the Galaxy. The amplitude of the corresponding proper motions

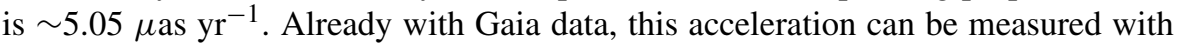
an accuracy of $\pm 0.35 \mu$ as $\mathrm{yr}^{-1}$ meaning a 10-sigma detection of the effect. From a combined optical-NIR solution the effect will be measured with 2 or 3 digits giving both much better stability of the resulting reference frame and a stringent constraint for the modelling of the galactic dynamics.

Another pattern in the proper motions of quasars comes from the instantaneous velocity of the Solar System with respect to the cosmological microwave background (CMB). This will cause extragalactic sources to undergo an apparent systematic proper motion. The effect is referred to as cosmological or parallactic proper motion [123]. This effect depends on the redshift, $z$, and fundamental cosmological parameters can in principle be determined from the motion. The velocity of the Solar System with respect to the rest frame of the observed Universe can be measured from a dipole pattern in the $\mathrm{CMB}$ temperature. Observations of the $\mathrm{CMB}$ indicate that $v=369 \pm 0.9 \mathrm{~km} \mathrm{~s}^{-1}$ in the apex direction with Galactic longitude $l=263.99^{\circ}$ and latitude $b=48.26^{\circ}[107,185]$. This motion should produce a parallactic shift of all extragalactic objects towards the antapex.

Both the acceleration of the Solar System and the cosmological proper motion give rise to dipole patterns in the proper motions of distant objects. However, the former does not depend on the redshift, while the latter does, which makes it possible to separate the effects. [16] showed that the cosmological proper motion effect is only just within the theoretical limits of the Gaia mission but the centroiding of extended Galaxies may result in the effect being too difficult to measure. However, improving the proper motions by a factor of 14-20 with a new astrometry mission 20 years later would bring such measurements safely within reach. Thus, at least for quasars with $z<1$ the Hubble law can be verified by the model-independent trigonometric parallaxes.

There are additional, rather speculative scenarios leading to additional patterns of proper motions in certain cosmologies. Kristian and Sachs [139] pointed out that an anisotropic Hubble expansion of the Universe would result in a certain quadrupole pattern of proper motions of quasars that could be a function of the redshifts and can be measured in principle. Quercellini et al. [193, 194] used the term cosmic parallax for the varying angular separation between any two distant sources, again caused by the anisotropic expansion of the Universe. Some models predict that anisotropy of 
the expansion cannot be constrained by the CMB measurements. The predicted sys-

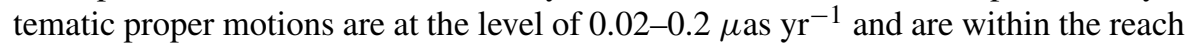
of the common Gaia-GaiaNIR astrometry that will be used to confirm or constrain these scenarios.

Finally, one more source of systematic proper motions is gravitational waves. It is well known that a gravitational wave passing through an astrometric observer causes shifts of observed position of all sources of the sky. Those shifts depend on the mutual orientation of the direction towards the source and the propagation direction of the gravitational wave as well as on time. This effect allows for an astrometric detection or constraints of gravitational waves [31, 101, 132, 190]. Two regimes for astrometric detection of gravitational waves should be distinguished [132]. The gravitational waves with periods longer than the time span covered by astrometric data (i.e., many years; these are primordial gravitational waves of cosmological nature) are absorbed by proper motions. One can show that the corresponding systematic proper motions of quasars are dominated by the quadrupole. Since the effect is indistinguishable from residual systematic errors in the solution, this allows only to give an upper estimate of the energy flux of primordial gravitational waves with all frequencies below a certain limit $[101,162]$ The current estimate from VLBI astrometry based on the data of [233] is $\Omega<0.009 \times H / 100 \mathrm{~km} / \mathrm{s} / \mathrm{Mpc}$ for frequencies below $1.5 \mathrm{nHz}$ [162]. Gaia is expected to improve this limit by a factor of 80 . The combined Gaia-GaiaNIR solution can improve Gaia results by a factor of up to 400 .

Another regime is the detection of higher-frequency gravitational waves from binary supermassive black holes. Recently, an algorithm for the detection was suggested [132] and is now being implemented for Gaia data. A new mission will give another improved opportunity to detect the signature of gravitational waves from astrometry. Compared to Gaia, the detection limit of GaiaNIR is improved both because of larger number of observed sources and the possibility to use significantly more accurate proper motions for the sources common to both missions.

Some additional tests of fundamental physics can be performed with GaiaNIR data which both provide a longer observational time span (combined with Gaia) and allow for accurate astrometry in optically obscured regions. A few examples here are:

- More accurate tests using the motion of asteroids in the weak field limit of the Solar System (PPN- $\gamma$ and PPN- $\beta$, time dependence of the gravitational constant).

- Strong field gravity tests at the Galactic Centre in the NIR (this includes both deflection of light in the gravitational field of the central Black Hole and the dynamics of the stars near the Galactic Centre).

\section{Photometry}

For a new NIR astrometry mission it is proposed that the photometry be implemented as filters deposited directly on the detector material forming 4 broad bands. This will provide photometry of all sources, sufficient for the chromatic corrections of astrometry and it will give photometry of narrow double stars and crowded areas 
which cannot be obtained from the ground nor can it be obtained with Gaia because the long prism photometer spectra of the two stars overlap. Ground-based surveys of multi-colour photometry and spectra will be available for astrophysical studies for a large fraction of the stars but Gaia-like precision and accuracy, in the mmag realm, is much better than any ground-based survey. There are several reasons to perform photometry:

- chromatic corrections of the astrometric observations;

- astrophysical information for all objects, including astrophysical classification for objects such as stars, quasars, etc.;

- astrophysical characterisation, e.g., interstellar reddening, effective temperatures of stars, photometric redshifts of quasars, etc.;

- photometric distances for those sources with large parallax relative error;

- multiwavelength variability of sources.

For a new NIR astrometry mission the first reason is still valid to the same extent as for Gaia, but the justification for the others has changed. By the time of a new NIR astrometry mission, multi-colour photometry will be available for many of the observed stars with better spectral resolution than the mission itself can provide. Such photometry will be available from large surveys such as Pan-STARRS and the Rubin Observatory, providing five or six spectral bands from 300 to $1100 \mathrm{~nm}$. However, both surveys have bright saturation limits at 15 mag and 17 mag, respectively, and their angular resolution cannot be much better than 0.5 arcsec since they are ground based. In addition, they do not cover the full sky. Instead, the new mission will be an all-sky survey and, very importantly, will be at the mmag accuracy level. For Gaia the angular resolution along scan of the astrometric observation is about 0.12 arcsec. For photometry the spectral dispersion limits the angular resolution for multiple stars and crowded regions as the spectra overlap, each spectrum having a length of 1-2 arcsec.

For the new mission, it is proposed to consider filter photometry which gives higher angular resolution in crowded fields due to far less blending and reduces the readout noise and the background contributions, so improving the accuracy at the faint limit. The two prisms on Gaia could be replaced by four filters as illustrated in Fig. 4. The first band must cover the complete wavelength (white light) to ensure all stars are detected while subsequent bands have a reduced wavelength coverage to provide photometry and calibration data as mentioned above. The filters themselves can be deposited directly onto the detector material to avoid complicating the telescope optics and there is no need for Sky Mapper (SM) detectors as the motion of the stars on the detectors is different for each Field-of-View (FoV) and this can be used to determine their origin and to track them across the focal plane. This new design was outlined in the ESA study report (https://sci.esa.int/s/8a65kZA) and helped to greatly reduce the complexity and hence cost of the focal plane assembly.

Detailed studies of the optimal filter bands need to be done for a mission proposal and the concept in Fig. 4 may be modified depending on a detailed trade-off between achieving accurate chromatic corrections and the possibility of getting excellent astrophysical information at the same time. 


\section{GaiaNIR Focal Plane}

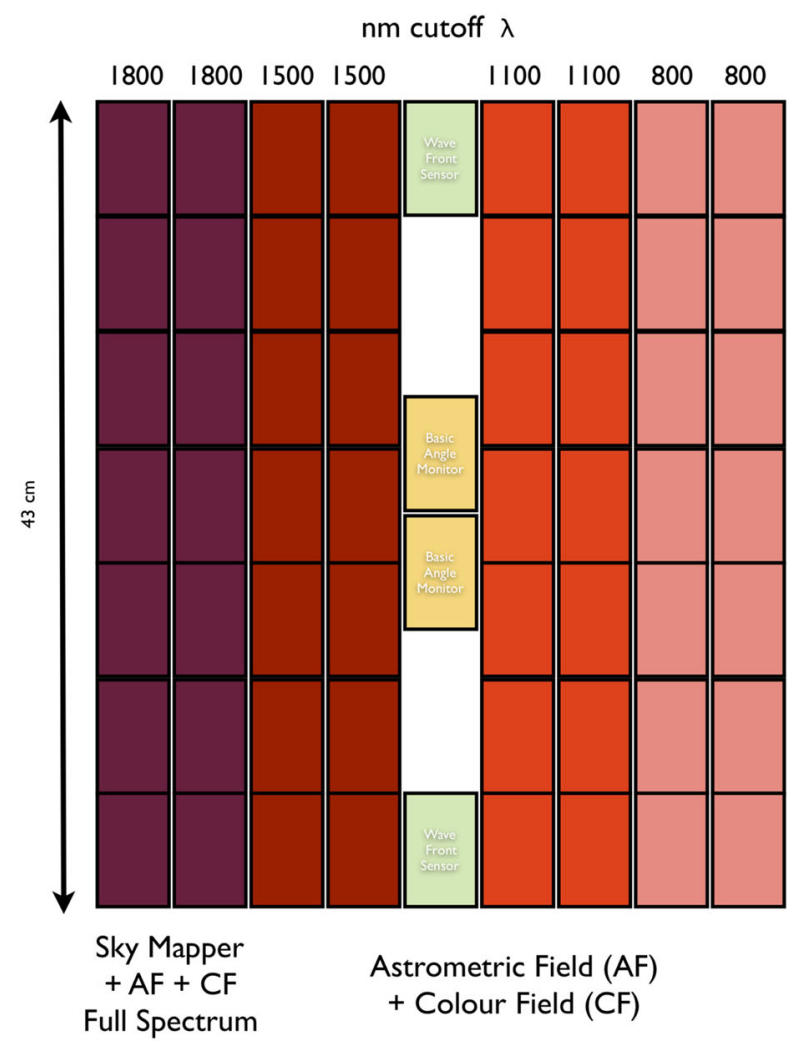

Fig. 4 Proposed focal plane array and filter bands used in the GaiaNIR CDF study (see page 203 in https://sci.esa.int/s/8a65kZA). The array consists of $60 \mathrm{NIR}$ detectors, arranged in 7 across-scan rows and 9 along-scan strips (out of which 8 are for the astrometric/photometric field, divided into 4 photometric fields (i.e. 4 different cut-off wavelengths each starting from $\sim 400 \mathrm{~nm}$ ). The new array is less than half the size of Gaia's

\subsection{HR diagrams and completeness}

The multi-band photometry from a new NIR astrometry mission, when combined with its precise parallax, enables the construction of various colour-magnitude diagrams (CMD) from these bands. The less extinction-limited CMDs from GaiaNIR are highly complementary to spectroscopic surveys in two ways: a) The star formation history of any stellar population can be well constrained by the number distribution of stars in the CMDs and better constraining the SFH is the key to better interpreting the chemical evolution of the Milky Way; and b) A precise CMD will put a strong prior on $\log g$ and effective temperature of the stars and, subsequently, improve the determination of elemental abundances of stars from stellar spectra. 
Galactic archaeology is propelled by the myriad of large-scale surveys. However, how well we can disentangle and measure subtle Galactic properties depends critically on our ability to model very precisely the selection function of these surveys what stars or populations do we miss, at what location, and at what ratio. Currently, most statistical modelling of the Milky Way relies on the 2MASS IR survey to set the completeness, but the 2MASS completeness cuts off around 16th magnitude. As future spectroscopic surveys such as MSE [232] intend to go deeper than that, having an all-sky, less extinction-limited, deep, NIR survey such as GaiaNIR will be instrumental for statistically modelling the Milky Way.

\subsection{Photometric distances}

Distances of stars are needed not only to derive their 3D location but to derive tangential velocities from proper motions as well. Distances may be obtained from the Gaia parallaxes, but they must be supplemented by photometric-spectroscopic distances for remote stars $([5,6,61,157]$, etc). The latter method will be much strengthened through calibration with accurate trigonometric distances from Gaia which will provide, e.g., better than 1 percent accuracy for 10 million stars, most of these will be dwarfs. For giants of luminosity class III, the sample within $2 \mathrm{kpc}$ (corresponding to a parallax of $500 \mu \mathrm{as}$ ) from the Sun will contain stars brighter than $\mathrm{V}=11.5$ if we assume $\mathrm{M}=0.0 \mathrm{mag}$ to be typical. With $\sigma_{\pi}=7.1 \mu$ as a relative accuracy better than $7.1 / 500$ or 1.4 percent is then obtained for distances of giants. More precise estimates of the number of stars may be obtained from the population synthesis Galaxy star count model TRILEGAL [94, 95] as described in Section 3.1. of [180].

It will be crucial for the determination of photometric distances to these stars that their absolute magnitudes can be accurately calibrated. The following studies of photometric calibration in clusters are available: [22, 238, 239] and [202]. An accuracy of $10 \%$ will perhaps be common by 2025 after calibration with Gaia data and an accuracy of $1 \%$ or $2 \%$ may even be obtained for giants and some other types of stars.

The uncertainties of photometry in Gaia DR2 are presented by [77] (in Figs. 9-11) and scaling to the end-of-mission, the photometric errors at $\mathrm{G}=19$ are about $3 \mathrm{mmag}$ for $\mathrm{G}$ and $10 \mathrm{mmag}$ for the low-dispersion red photometer $\left(G_{\mathrm{RP}}\right)$, but much larger for the blue band photometer $\left(G_{\mathrm{BP}}\right)$. Absolute magnitudes may therefore be obtained from the spectra of faint red stars [110]. The main source for accurate absolute magnitudes of faint Gaia stars, after luminosity calibration with the Gaia parallaxes, would be the many photometric and spectroscopic surveys. Examples include 4MOST [62], WEAVE [60], MOONS [57], APOGEE I-II [149], LAMOST [251].

\subsection{Inferred chemical abundances}

Almost every object will have its position on the Kiel and HR diagrams determined with superb accuracy. However, chemistry will be missing, except for a relatively small fraction of objects which will be targeted by ground-based spectroscopic surveys. But knowledge of effective temperature, luminosity and surface gravity allows 
us to play a classical game for estimation of chemical abundances of $[\mathrm{Fe} / \mathrm{H}],[\alpha / \mathrm{Fe}]$, and beyond: by using either narrow-band photometric filters centred on strong NIR lines of individual elements (and on the continuum) or using very low resolution spectrophotometry, as done with Gaia's $G_{\mathrm{BP}}$ and $G_{\mathrm{RP}}$ bands. Studies of this concept are currently ongoing for ESA [253] in the context of the Gaia mission where it is being tested on real stars (using Gaia parallaxes, chemistry and parameters derived by the Galah ground-based survey [41]). Note that ground-based spectroscopic surveys are crucial for providing a training set (comprised of millions of stars) which can then form a base for the billions of sources using suitable artificial intelligence techniques (e.g. The Cannon, [176]). If successful, this approach can be reformulated for the NIR (e.g. K-band) and is potentially very interesting.

\section{Synergies with other surveys}

In order to understand how our science case complements other surveys and instruments in the coming decades we briefly review the main relevant ground-based surveys currently ongoing and coming into operation. We also consider specific spectroscopic instruments which will provide complementary radial velocity measurements and finally we will consider synergies between the new astrometric mission with some forthcoming space telescope missions currently under preparation. The discussion here is limited to the most obvious projects as new instruments and surveys are being proposed as we write.

\subsection{Ground based surveys and spectrographs}

- SDSS: The Sloan Digital Sky Survey has been one of the most-successful and influential surveys in the history of astronomy, creating three-dimensional maps with deep multi-colour images of one third of the sky, and spectra for more than three million astronomical objects. SDSS-V [133] is unique among spectroscopic surveys in that it is NIR, optical and all-sky, with infrastructures in both hemispheres giving a multi-epoch spectroscopic survey of over six million objects. It is designed to decode the history of the Milky Way, trace the emergence of the chemical elements, reveal the inner workings of stars, and investigate the origin of planets. It will create an integral-field spectroscopic map of the gas in the Galaxy and the Local Group that is a thousand times larger than the current state of the art. SDSS-V's program (2020-2025) will be well-timed to coincide with other major space missions (e.g., TESS, Gaia, eROSITA, PLATO) and groundbased projects. There are clear synergies between a new all-sky NIR mission and SDSS-V's Milky Way Mapper (MWM) which is a multi-object spectroscopic survey to obtain NIR and/or visible spectra of more than 4 million stars.

- The Rubin Observatory: The Rubin Observatory (previously the Large Synoptic Survey Telescope) [116] is a large, wide-field ground-based system designed to obtain deep images covering the sky visible from northern Chile. The telescope will have an $8.4 \mathrm{~m}$ (6.5 m effective) primary mirror, a $9.6 \mathrm{deg}^{2}$ field of view, a 3.2 
gigapixel camera, and six filters (u,g,r,i,z,y) covering the wavelength range 320$1050 \mathrm{~nm}$. Taking repeated images of the sky every few nights in multiple bands for ten years will result in an astronomical catalogue thousands of times larger than previous ones. The project will address: What is the mysterious dark energy that is driving the acceleration of the cosmic expansion? What is dark matter, how is it distributed, and how do its properties affect the formation of stars, galaxies, and larger structures? How did the Milky Way form, and how has its present configuration been modified by mergers with smaller bodies over cosmic time? What is the nature of the outer regions of the Solar System? Is it possible to make a complete inventory of smaller bodies in the Solar System, especially potentially hazardous asteroids that could someday impact the Earth? Are there new exotic and explosive phenomena in the universe that have not yet been discovered? A new NIR mission would broaden the wavelength range and provide much more accurate astrometry down to $\mathrm{H}<20$ magnitude. The Rubin Observatory will do good astrometry but with limited accuracy from the ground, obtaining parallax and proper-motion measurements of comparable accuracy, $\sigma_{\pi} \sim 0.6$ mas, $\sigma_{\mu} \sim$ 0.2 mas $\mathrm{yr}^{-1}$, to those of Gaia at its faint limit $(\mathrm{G}<20)$ and smoothly extend the error versus magnitude curve deeper by about 5 mag [115]. Thus, the Rubin Observatory astrometric project is anchored in the successful Gaia mission and is extremely complementary to GaiaNIR.

- Pan-STARRS is a wide-field imaging system operated by the Institute for Astronomy at the University of Hawaii. The survey uses a $1.8 \mathrm{~m}$ telescope and its 1.4 gigapixel camera to image the sky in five broadband filters ( $\mathrm{g}, \mathrm{r}, \mathrm{i}, \mathrm{z}, \mathrm{y})$ sensitive from 400-1100 nm [234]. By detecting differences from previous observations of the same areas, Pan-STARRS is discovering a large number of new asteroids, comets, variable stars, supernovae and other celestial objects. Its primary mission is now to detect Near-Earth Objects that threaten impact events and it is expected to create a database of all objects visible from Hawaii (three quarters of the entire sky) down to apparent magnitude 24. Similar synergies with a new NIR mission apply here.

- VVV: ESO's NIR variability survey [167] is scanning the Milky Way bulge and an adjacent section of the mid-plane where star formation activity is high. The survey uses the 4-metre VISTA telescope during five years, covering $\sim 10^{9}$ point sources across an area of $520 \mathrm{deg}^{2}$, including 33 known globular clusters and $\sim 350$ open clusters. The final product is a deep NIR atlas in five passbands $(900-2500 \mathrm{~nm})$ and a catalogue of more than $10^{6}$ variable point sources. Unlike single-epoch surveys that, in most cases, only produce 2-D maps, the VVV variable star survey will enable the construction of a 3-D map of the surveyed region using distance indicators (albeit with potentially unknown biases [148]) such as RR-Lyrae stars, and Cepheids, yielding important information on the ages of the populations. An extended survey (VVVX) is ongoing. Observation data will be combined from many sources including Gaia to better understand variable sources in the inner Milky Way, globular cluster evolution, a population census of the Galactic bulge and centre, as well as studies of star-forming regions in the disc. By combining VVV, whose accuracy is currently around $0.7 \mathrm{mas} \mathrm{yr}^{-1}$ 
[218], and the precise distances from a new NIR astrometry mission one would get more precision in extinction and distances in the most difficult and unexplored regions of the Milky Way disc which would also help to calibrate any biases in the VVV distance indicators.

- 4MOST is the ESO's wide-field, high-multiplex (1500-3000 fibres) spectrograph (see Fig. 1 [62]) which will be running nearly full time on the $4 \mathrm{~m}$-class telescope (VISTA) from about 2022, and capable of providing radial velocity, stellar parameters and chemical composition for a large number of faint stars. Radial velocity accuracies of $\leq 2 \mathrm{~km} \mathrm{~s}^{-1}$ for the faintest stars observed by Gaia, which will be also observed in a new NIR astrometry mission, may be obtained with 4MOST, which will provide the full 3-D space velocities for unambiguous dynamical studies as well as chemistry for more than 10 million stars [26, 56].

- WEAVE [60] is a new wide-field multi-object spectrograph for the William Herschel Telescope that will do optical ground-based follow up of the LOFAR and Gaia surveys. WEAVE will host 1000 multi-object fibres which are fed to a single spectrograph, with a pair of cameras. The project is now being developed with commissioning in 2020 and science operations starting in early 2021. Unfortunately, 4MOST and WEAVE will only operate in the wavelength region 390-1000 nm and 370-1000 nm respectively and will not probe the NIR to open new science cases in the Galactic disc and the Galactic Centre region.

- MOONS: Multi-Object Optical and NIR Spectrograph [57] on the Very Large Telescope (VLT) $8.2 \mathrm{~m}$ telescope will operate in the wavelength range 800$1800 \mathrm{~nm}$. The large collecting area offered by the VLT, combined with the large multiplex and wavelength coverage will constitute a powerful, unique instrument able to pioneer a wide range of Galactic, extragalactic, and cosmological studies and provide follow-up for major facilities such as Gaia. MOONS is capable of providing radial velocity and chemical compositions for faint stars for a new NIR astrometry mission.

- APOGEE-I \& II [149], part of the SDSS survey, operates from 1510-1700 nm using high-resolution, high signal-to-noise IR spectroscopy to penetrate the dust that obscures the disc and bulge of our Galaxy to survey over 400,000 red giant stars across the full range of the Galactic bulge, bar, disc, and halo. By the end of the survey in 2020 APOGEE will provide a catalogue of 600,000 objects in both hemispheres. Combining APOGEE data with Gaia is already having a major impact but this is just the tip of the iceberg as the statistics are low both because the number of targets are still low, and because Gaia is not NIR. APOGEE will yield $\sim 0.1 \mathrm{~km} / \mathrm{s}$ accuracy radial velocities as well as chemical information for over 15 elements.

- Subaru Prime Focus Spectrograph (PFS; [229]) is a massively multiplexed fiberfed optical and NIR three-arm spectrograph (Nfiber $=2400,380 \leq \lambda \leq 1260$ $\mathrm{nm}, 1.3$ degree diameter field of view). Data will be secured for $10^{6}$ stars in the Galactic thick-disc, halo, and tidal streams as faint as $\mathrm{V} \sim 22$, including stars with $\mathrm{V}<20$ to complement the goals of the Gaia mission. A mediumresolution mode with $\mathrm{R}=5000$ to be implemented in the red arm will allow the measurement of multiple $\alpha$-element abundances and more precise velocities for Galactic stars. 
- The Large Sky Area Multi-Object Fiber Spectroscopic Telescope (LAMOST; [251]) survey contains two main parts: the LAMOST ExtraGAlactic Survey (LEGAS), and the LAMOST Experiment for Galactic Understanding and Exploration (LEGUE) survey of Milky Way stellar structure. The unique design of LAMOST enables it to take 4000 spectra in a single exposure to a limiting magnitude as faint as $\mathrm{r}=19$ at the resolution $\mathrm{R}=1800$. This telescope therefore has great potential to efficiently survey a large volume of space for stars and galaxies.

- And other surveys are coming online, a few examples include: The Maunakea Spectroscopic Explorer (MSE) [232] is an end-to-end science platform for the design, execution and scientific exploitation of spectroscopic surveys; SpecTel [74] is a proposed 10-12 m class Spectroscopic Survey Telescope which is being designed to access a larger fraction of objects from Gaia, the Rubin Observatory, Euclid, and WFIRST than any currently funded or planned spectroscopic facility.

\subsection{Space missions}

- WFIRST: The Wide Field Infrared Survey Telescope [226] is a NASA observatory which will perform galaxy surveys over thousands of square degrees for dark energy weak lensing and baryon acoustic oscillation measurements and will monitor a few square degrees for dark energy SN Ia studies. It will perform microlensing observations of the Galactic bulge for an exoplanet census and direct imaging observations of nearby exoplanets with a pathfinder coronagraph. WFIRST has a $2.4 \mathrm{~m}$ telescope, the same size as Hubble's, but with a FoV 100 times greater. WFIRST is slated for launch in the mid-2020s although it faces repeated funding challenges. The wide field instrument includes filters that provide an imaging mode covering 480-2000 nm, and two slit-less spectroscopy modes covering 1000-1930 nm with resolving power 450-850, and 800-1800 nm with resolving power of 70-140. The instrument provides a sharp point spread function, precision photometry, and stable observations for implementing the dark energy, exoplanet microlensing, and NIR surveys. WFIRST is not an all-sky survey mission but is extremely complementary to Gaia and the JWST and could act as a source of first epoch measurements for a new NIR astrometry mission in a similar manner to small-JASMINE (see below).

- The Euclid mission [2] will have very high quality NIR photometry but its science cases are focused on the extragalactic sky which is presently defined by the regions covering $|b|>30^{\circ}$ which precisely avoids those regions which would be most interesting for NIR Galactic astronomy. Euclid measurements of objects out of the Galactic plane could act as second epoch measurements for Gaia and for very red faint objects, not already in Gaia, could provide first epoch measurements for a new NIR astrometry mission.

- JWST: The James Webb Space Telescope [175] has a NIR (600-2500 nm) instrument called NIRCam with 32 mas pixels. Combined with the telescope's $6.5 \mathrm{~m}$ segmented primary mirror, NIRCam images will have an angular resolution similar to what is achieved by the HST. Thus, JWST will have the potential for astrometric performance similar to that achieved with HST imaging. Using archived data from HST observations to provide first epoch positions, JWST 
will measure proper motions of stars within globular clusters and other crowded fields. JWST can also provide parallaxes for nearby objects too faint (e.g. neutron stars, L-dwarfs) for Gaia. As an example of the usefulness of all-sky astrometry the Gaia astrometric catalog is being included in the HST Guide Star Catalog (GSC), the Hubble Source Catalog (HSC) and the JWST calibration field catalog in order to improve their astrometric accuracy [205], and similar applications of a new astrometry mission will surely arise. As with WFIRST the JWST can be used to provide first epoch measurements in small regions of the sky. However, a new all-sky NIR mission would provide astrometric measurements with slightly lower resolution all over the sky!

- LISA: The Laser Interferometer Space Antenna [1] is a mission led by ESA to detect and accurately measure gravitational waves from astronomical sources. LISA would be the first dedicated space-based gravitational wave detector. Ultrashort period Galactic double white dwarf binaries are unique multi-messenger tracers of the Milky Way. They can be detected in large numbers through electromagnetic radiation by Gaia and through gravitational waves by the upcoming LISA mission [135]. The synergies between Gaia and LISA observations of double white dwarf binaries would allow the study of the Milky Way baryonic structure due to LISA's ability to localise binaries through virtually the whole Galactic plane while Gaia yields information on their motion; tracing the underlying total enclosed mass. However, many of the objects detected by LISA will be in regions that are hardly accessible to visible observations such as the inner part of the Galactic disc, the bulge, and beyond [136]. Of course such synergies can be improved if a new NIR astrometry mission can partially see into the disc and centre of the Galaxy where many of the double white dwarfs are found.

- PLATO [147, 161, 196], TESS [200] and beyond: There are also synergies with the many upcoming exoplanet surveys, generally space astrometry addresses very long period massive exoplanets, while other transit and radial velocity exoplanet surveys are aimed at shorter period exoplanets. We have shown using numerical studies (see Section 3.14) that the combination of two missions with a 20 year gap can yield exoplanets with periods of 30-40 years allowing the coplanarity of these systems to be studied with multi-mission data. Therefore, a new astrometry mission will be very complementary for exoplanet science particularly as astrometry recovers all seven orbital parameters for the exoplanet as well as for the science being made with asteroseismology for Galactic archaeology [161].

- Small-JASMINE [250] is being developed by the National Astronomical Observatory of Japan, Japanese universities, and ISAS/JAXA in collaboration with ESA, Gaia-DPAC, and the US Naval Observatory. The aim is to make astrometric measurements in the Hw-band (1100-1700 nm). Small-JASMINE will use the Gaia visible reference frame to do relative plate overlap astrometry in a radius of 0.7 degrees or $\sim 100 \mathrm{pc}$ around the bulge and Galactic Centre and selected directions towards interesting objects such as star-forming regions and/or M-dwarf exoplanet transit follow-up. The mission will make high frequency measurements (100 minutes) with astrometry accuracy of $\sim 25 \mu \mathrm{as}\left(\mathrm{yr}^{-1}\right)$ for bright stars $(\mathrm{Hw}<12.5 \mathrm{mag})$ and $\sim 125 \mathrm{mas}\left(\mathrm{yr}^{-1}\right)$ for fainter stars $(\mathrm{Hw}<15 \mathrm{mag})$. 
Small-JASMINE will be very complementary to Gaia providing proper motions for about a hundred thousand bulge stars allowing the astrophysics of the Galactic Centre to be studied. The mission is due to be launched by the mid2020's and can be seen as a precursor by providing first epoch measurements, albeit very limited, for an all-sky visible and NIR survey. The combination of Small-JASMINE-GaiaNIR in the Galactic nuclear bulge region would be a very powerful tool.

The combination of multi-messenger data from these surveys and instruments would greatly complement a new space astrometry mission in the NIR in many cases providing first epoch measurements. The spectroscopic instruments would provide the third dimension of the space velocities needed particularly in the dusty regions that currently cannot be explored by Gaia and at first sight it would seem unnecessary to include a spectrograph in a new mission design. However, a new NIR astrometry mission could provide spectra for possibly billions of objects (to some limiting magnitude) in the visible and NIR globally on the sky, albeit at low resolution, which would provide this third component of the space velocity for many observed objects compared to some tens of millions of spectra from selected areas with different instruments. A new mission would potentially provide a unique opportunity to obtain photometry and spectroscopy with a single instrument making the calibration of the science results uniform across the sky. However, we have concluded that the spectroscopic option for a new mission is a nice addition (presumably at considerable cost) but is not a necessary requirement to achieve the science goals outlined in this document.

In this context it is worth noting that the advent of Gaia led to the community organizing facilities for Gaia follow-up (see the ESA-ESO WG document) from where projects like 4MOST, WEAVE, and the Milky Way Mapper project in SDSS-V were born. We can thus expect the astronomy community to undertake similar efforts to organize a massive NIR spectroscopic follow-up should GaiaNIR become a reality.

\section{Mission scenarios}

As discussed in the introduction we see international collaboration as the best way to achieve the science goals outlined here while keeping within the M-class budget constraints of ESA, so we would welcome collaboration with the US, Australia, and/or Japan. The US is the world leader in detector technology particularly for the NIR so a collaboration with the US makes a lot of sense. We have recently submitted an APC white paper [109] to the ASTRO 2020 call outlining the state of the profession considerations on detector development for our application, which may also be generally applicable to astronomy, remote sensing, planetary observation and for LIDAR applications. In the APC white paper four different technologies have been identified which could meet our needs provided sufficient market motivation and investment in them can be found. Here we make a clear statement that it is possible to have visible-NIR detectors with TDI-like operation but their development will come with a financial cost. 
1. A hybrid solution which uses a HgCdTe NIR detector layer bump bonded to a Si CCD. The idea is that the photons are detected in the surface NIR layer and transferred to the Si buried channel at each pixel. Charge can then be easily moved along the pixels in sync with the charge generation, thus achieving TDI. What is not known yet is how efficiently the charge can be transferred from the NIR detection layer to the Si CCD and if both materials can be operated at the same temperature in a space environment making this development potentially complex.

2. Using HgCdTe Avalanche Photodiodes (APDs) with TDI-like signal processing capability. The challenge here is to scale the existing technology to larger format arrays and ensure the dark current does not introduce unwanted noise at temperatures above $100 \mathrm{~K}$. The Australia National University working with Leonardo (Italy) are actively developing this technology for large-format astronomy applications and are keen to be involved. The development effort required for these devices does not seem excessive.

3. Ge detectors due to the lower band gap can detect NIR radiation of longer wavelengths than possible with Si detectors. Clearly this technology is new but many of the manufacturing techniques developed for $\mathrm{Si}$ are also applicable to Ge and further development is needed to see if they can be used for low noise and visible-NIR capabilities in large format arrays. The wavelength range is, however, limited to $1600 \mathrm{~nm}$.

4. Microwave Kinetic Inductance Detectors (MKIDs) are cooled, multispectral, single photon counting, multiplexed devices capable of observation in the UV through to mid-IR. They measure the energy of each photon to within several percent and $\log$ the time of arrival to within 2 microseconds, making them ideal for TDI-like operation. Whilst relatively new, small MKID arrays (several thousand pixels) have already been utilised on ground-based telescopes but moving towards gigapixel space-based devices is very challenging given that they also require active cooling which is undesirable.

The use of visible-NIR TDI capable detectors is optimal for maximising the science return by reusing the same well established concepts as Gaia. A new NIR astrometry mission will detect at least 5 times as many stars and open up the important NIR regions of the Galaxy. While the spacecraft is scanning outside the Galactic plane it would be possible to go deeper than $G=20.7$ improving the science return in the Halo region. The main challenge for this exciting science mission is clearly the development of the new detector technology at a reasonable cost.

Funding Open access funding provided by Lund University.

Open Access This article is licensed under a Creative Commons Attribution 4.0 International License, which permits use, sharing, adaptation, distribution and reproduction in any medium or format, as long as you give appropriate credit to the original author(s) and the source, provide a link to the Creative Commons licence, and indicate if changes were made. The images or other third party material in this article are included in the article's Creative Commons licence, unless indicated otherwise in a credit line to the material. If material is not included in the article's Creative Commons licence and your intended use is not permitted by statutory regulation or exceeds the permitted use, you will need to obtain permission directly from the copyright holder. To view a copy of this licence, visit http://creativecommonshorg/licenses/by/4.0/. 


\section{References}

1. Amaro-Seoane, P., Aoudia, S., Babak, S., Binétruy, P., Berti, E., Bohé, A., Caprini, C., Colpi, M., Cornish, N.J., Danzmann, K.: eLISA: Astrophysics and cosmology in the millihertz regime. GW Notes 6, 4-110 (2013). 1201.3621

2. Amendola, L., Appleby, S., Bacon, D., Baker, T., Baldi, M., Bartolo, N., Blanchard, A., Bonvin, C., Borgani, S., Branchini, E., Burrage, C., Camera, S., Carbone, C., Casarini, L., Cropper, M., de Rham, C., Di Porto, C., Ealet, A., Ferreira, P.G., Finelli, F., García-bellido, J., Giannantonio, T., Guzzo, L., Heavens, A., Heisenberg, L., Heymans, C., Hoekstra, H., Hollenstein, L., Holmes, R., Horst, O., Jahnke, K., Kitching, T.D., Koivisto, T., Kunz, M., La Vacca, G., March, M., Majerotto, E., Markovic, K., Marsh, D., Marulli, F., Massey, R., Mellier, Y., Mota, D.F., Nunes, N.J., Percival, W., Pettorino, V., Porciani, C., Quercellini, C., Read, J., Rinaldi, M., Sapone, D., Scaramella, R., Skordis, C., Simpson, F., Taylor, A., Thomas, S., Trotta, R., Verde, L., Vernizzi, F., Vollmer, A., Wang, Y., Weller, J., Zlosnik, T.: Cosmology and fundamental physics with the euclid satellite. Living Rev. Relativit. 16(1), 6 (2013). https://doi.org/10.12942/lrr-2013-6, arXiv:1206.1225

3. Anders, F., Chiappini, C., Minchev, I., Miglio, A., Montalbán, J., Mosser, B., Rodrigues, T.S., Santiago, B.X., Baudin, F., Beers, T.C., da Costa, L.N., García, R.A., García-Hernández, D.A., Holtzman, J., Maia, M.A.G., Majewski, S., Mathur, S., Noels-Grotsch, A., Pan, K., Schneider, D.P., Schultheis, M., Steinmetz, M., Valentini, M., Zamora, O.: Red giants observed by CoRoT and APOGEE: The evolution of the Milky Way's radial metallicity gradient. A\&A 600:A70 DOI10.1051/0004-6361/201629363 arXiv:1608.04951 (2017a)

4. Anders, F., Chiappini, C., Rodrigues, T.S., Miglio, A., Montalbán, J., Mosser, B., Girardi, L., Valentini, M., Noels, A., Morel, T., Johnson, J.A., Schultheis, M., Baudin, F., de Assis Peralta, R., Hekker, S., Themeß1, N., Kallinger, T., Garcí, R.A., Mathur, S., Baglin, A., Santiago, B.X., Martig, M., Minchev, I., Steinmetz, M., da Costa, L.N., Maia, M.A.G., Allende Prieto, C., Cunha, K., Beers, T.C., Epstein, C., García pérez, A.E., García-hernández, D.A., Harding, P., Holtzman, J., Majewski, S.R., Mészáros, S., Nidever, D., Pan, K., Pinsonneault, M., Schiavon, R.P., Schneider, D.P., Shetrone, M.D., Stassun, K., Zamora, O., Zasowski, G.: Galactic archaeology with asteroseismology and spectroscopy: Red giants observed by CoRoT and APOGEE. A\&A 597, A30 (2017b). https://doi.org/10.1051/0004-6361/201527204 arXiv:1604.07763

5. Anders, F., Queiroz, A.B., Chiappini, C., Santiago, B.X., Fernández-Trincado, J.G., Meza, A., SDSS-IV/APOGEE Collaboration: The DR14 APOGEE-TGAS catalogue: Precise chemokinematics in the extended solar vicinity. In: Chiappini, C., Minchev, I., Starkenburg, E., Valentini, M. (eds.) Rediscovering Our Galaxy, IAU Symposium, vol. 334, pp. 153-157 (2018). https://doi.org/10.1017/S1743921317008882, arXiv:1708.09319

6. Anders, F., Khalatyan, A., Chiappini, C., Queiroz, A.B., Santiago, B.X., Jordi, C., Girardi, L., Brown, A.G.A., Matijevič, G., Monari, G., Cantat-Gaudin, T., Weiler, M., Khan, S., Miglio, A., Carrillo, I., Romero-Gómez, M., Minchev, I., de Jong, R.S., Antoja, T., Ramos, P., Steinmetz, M., Enke, H.: Photo-astrometric distances, extinctions, and astrophysical parameters for Gaia DR2 stars brighter than $\mathrm{G}=18$. A\&A 628, A94 (2019). https://doi.org/10.1051/0004-6361/201935765

7. Anderson, R.I., Saio, H., Ekström, S., Georgy, C., Meynet, G.: On the effect of rotation on populations of classical Cepheids. II. Pulsation analysis for metallicities 0.014, 0.006, and 0.002. A\&A 591, A8 (2016). https://doi.org/10.1051/0004-6361/201528031, arXiv:1604.05691

8. Andrews, J.J., Chanamé, J., Agüeros, M.A.: Wide binaries in Tycho-Gaia: search method and the distribution of orbital separations. MNRAS 472(1), 675-699 (2017). https://doi.org/10.1093/mnras/stx 2000, arXiv:1704.07829

9. Andrews, J.J., Chanamé, J., Agüeros, M.A.: Wide binaries in Tycho-Gaia II: metallicities, abundances and prospects for chemical tagging. MNRAS 473(4), 5393-5406 (2018). DOI10.1093/mnras/stx2685, arXiv:1710.04678

10. Andrews, J.J., Anguiano, B., Chanamé, J., Agüeros, M.A., Lewis, H.M., Hayes, C.R., Majewski, S.R.: Using APOGEE wide binaries to test chemical tagging with dwarf stars. ApJ 871(1), 4 (2019a). https://doi.org/10.3847/1538-4357/aaf502, arXiv:1811.12032

11. Andrews, J.J., Breivik, K., Chatterjee, S.: Weighing the darkness: Astrometric mass measurement of hidden stellar companions using gaia. ApJ 886(1), 68 (2019b). https://doi.org/10.3847/1538-4357/ ab441f, arXiv:1909.05606 
12. Antoja, T., Helmi, A., Romero-Gómez, M., Katz, D., Babusiaux, C., Drimmel, R., Evans, D.W., Figueras, F., Poggio, E., Reylé, C., Robin, A.C., Seabroke, G., Soubiran, C.: A dynamically young and perturbed Milky Way disk. Nature 561(7723), 360-362 (2018a). https://doi.org/10.1038/s41586018-0510-7, arXiv:1804.10196

13. Antoja, T., Helmi, A., Romero-Gómez, M., Katz, D., Babusiaux, C., Drimmel, R., Evans, D.W., Figueras, F., Poggio, E., Reylé, C., Robin, A.C., Seabroke, G., Soubiran, C.: A dynamically young and perturbed Milky Way disk. Nature 561(7723), 360-362 (2018b). https://doi.org/10.1038/s41586018-0510-7, arXiv:1804.10196

14. Aumer, M., Schönrich, R.: Origin of the high $\mathrm{v}_{l o s}$ feature in the Galactic bar. MNRAS 454(3), 3166-3184 (2015). https://doi.org/10.1093/mnras/stv2252, arXiv:1507.00907

15. Baba, J., Kawata, D., Matsunaga, N., Grand, R.J.J., Hunt, J.A.S.: Gaia DR1 evidence of disrupting the perseus arm. ApJ 853(2), L23 (2018). https://doi.org/10.3847/2041-8213/aaa839, arXiv: 1712.04714

16. Bachchan, R.K., Hobbs, D., Lindegren, L.: Gaia reference frame amid quasar variability and proper motion patterns in the data. A\&A 589, A71 (2016). https://doi.org/10.1051/0004-6361/201527935, arXiv: 1512.08917

17. Bailer-Jones, C.A.L., Smith, K.W.: Finding rare objects and building pure samples: Probabilistic quasar classification with Gaia. In: Bailer-Jones, C.A.L. (ed.) American Institute of Physics Conference Series, American Institute of Physics Conference Series, vol. 1082, pp. 3-8 (2008). https://doi.org/10.1063/1.3059079, arXiv:0809.3373

18. Banik, I., Zhao, H.: Testing gravity with wide binary stars like $\alpha$ Centauri. MNRAS 480(2), 26602688 (2018). https://doi.org/10.1093/mnras/sty2007, arXiv:1805.12273

19. Barbuy, B., Chiappini, C., Gerhard, O.: Chemodynamical history of the galactic bulge. Annual Rev Astron Astrophys 56(1), 223-276 (2018). https://doi.org/10.1146/annurev-astro-081817-051826

20. Bastian, N., Covey, K.R., Meyer, M.R.: A universal stellar initial mass function? a critical look at variations. ARA\&A 48, 339-389 (2010). https://doi.org/10.1146/annurev-astro-082708-101642, arXiv:1001.2965

21. Bastian, U.: Direct detection of the sun's galactocentric acceleration. In: Perryman, M.A.C., van Leeuwen, F. (eds.) Future Possibilities for Astrometry in Space, vol. 379, p. 99. ESA Special Publication (1995)

22. Bellazzini, M., Ferraro, F.R., Pancino, E.: A new calibration of the RGB Tip as a Standard Candle. In: Lejeune, T., Fernandes, J. (eds.) Observed HR Diagrams and Stellar Evolution, Astronomical Society of the Pacific Conference Series, vol. 274, p. 288 (2002). arXiv:astro-ph/0110645

23. Belokurov, V., Erkal, D., Evans, N.W., Koposov, S.E., Deason, A.J.: Co-formation of the disc and the stellar halo. MNRAS 478(1), 611-619 (2018). https://doi.org/10.1093/mnras/sty982, arXiv: 1802.03414

24. Belokurov, V., Deason, A.J., Erkal, D., Koposov, S.E., Carballo-Bello, J.A., Smith, M.C., Jethwa, P., Navarrete, C.: The Pisces Plume and the Magellanic Wake. MNRAS L102, https://doi.org/10.1093/mnrasl/slz101, arXiv:1904.07909 (2019)

25. Benjamin, R.A., Churchwell, E., Babler, B.L., Indebetouw, R., Meade, M.R., Whitney, B.A., Watson, C., Wolfire, M.G., Wolff, M.J., Ignace, R.: First GLIMPSE results on the stellar structure of the galaxy. ApJ 630(2), L149-L152 (2005). https://doi.org/10.1086/491785, arXiv:astro-ph/0508325

26. Bensby, T., Bergemann, M., Rybizki, J., Lemasle, B., Howes, L., Kovalev, M., Agertz, O., Asplund, M., Barklem, P., Battistini, C., Casagrande, L., Chiappini, C., Church, R., Feltzing, S., Ford, D., Gerhard, O., Kushniruk, I., Kordopatis, G., Lind, K., Minchev, I., McMillan, P., Rix, H.W., Ryde, N., Traven, G.: 4MOST consortium survey 4: Milky Way disc and bulge high-resolution survey (4MIDABLE-HR). Messenger 175, 35-38 (2019). https://doi.org/10.18727/0722-6691/5123, arXiv: 1903.02470

27. Bird, J.C., Kazantzidis, S., Weinberg, D.H.: Radial mixing in galactic discs: the effects of disc structure and satellite bombardment. MNRAS 420(2), 913-925 (2012). https://doi.org/10.1111/j.13652966.2011.19728.x, arXiv:1104.0933

28. Bland-Hawthorn, J., Gerhard, O.: The Galaxy in Context: Structural, Kinematic and Integrated Properties. arXiv:1602.07702 (2016)

29. Blommaert, J.A.D.L., Groenewegen, M.A.T., Justtanont, K., Decin, L.: On the detection of CO and mass-loss of bulge OH/IR stars. MNRAS 479(3), 3545-3562 (2018). https://doi.org/10.1093/mnras/ sty1663, arXiv:1806.09603 
30. Bono, G., Dall'Ora, M., Fabrizio, M., Crestani, J., Braga, V.F., Fiorentino, G., Altavilla, G., Botticella, M.T., Calamida, A., Castellani, M., Catelan, M., Chaboyer, B., Chiappini, C., Clarkson, W., Contreras Ramos, R., Creevey, O., da Silva, R., Debattista, V., Degl'Innocenti, S., Ferraro, I., Gilligan, CK., Gonzalez, O., Hambleton, K., Iannicola, G., Inno, L., Kunder, A., Lemasle, B., Magrini, L., Magurno, D., Marconi, M., Marengo, M., Marinoni, S., Marrese, PM., Martinez-Vazquez, CE., Matsunaga, N., Monelli, M., Prada Moroni, PG., Musella, I., Navarro, MG., Neeley, J., Nonino, M., Pietrinferni, A., Pulone, L., Rich, MR., Ripepi, V., Sacco, G., Saha, A., Salaris, M., Sneden, C., Stetson, PB., Street, RA., Szabo, R., Tantalo, M., Tognelli, E., Torelli, M., Valenti, E., Walker, A., Zoccali, M.: unVEil the darknesS of The gAlactic buLgE (VESTALE). arXiv:1812.03124 (2018)

31. Book, L., Flanagan, E.: Astrometric effects of a stochastic gravitational wave background. In: APS Meeting Abstracts, p G12.004 (2011)

32. Bovy, J.: Detecting the disruption of dark-matter halos with stellar streams. Physic. Rev. Lett. 116(12), 121301 (2016). https://doi.org/10.1103/PhysRevLett.116.121301, arXiv:1512.00452

33. Bovy, J., Bahmanyar, A., Fritz, T.K., Kallivayalil, N.: The shape of the inner milky way halo from observations of the Pal 5 and GD-1 Stellar Streams. ApJ 833(1), 31 (2016a). https://doi.org/10.3847/ 1538-4357/833/1/31, arXiv:1609.01298

34. Bovy, J., Rix, H.W., Green, G.M., Schlafly, E.F., Finkbeiner, D.P.: On galactic density modeling in the presence of dust extinction. ApJ 818, 130 (2016b). https://doi.org/10.3847/0004-637X/818/2/130, arXiv:1509.06751

35. Bovy, J., Leung, H.W., Hunt, J.A.S., Mackereth, J.T., Garcia-Hernandez, D.A., Roman-Lopes, A.: Life in the fast lane: A direct view of the dynamics, formation, and evolution of the Milky Way's bar. arXiv:1905.11404 (2019)

36. Boylan-Kolchin, M., Weisz, D.R., Bullock, J.S., Cooper, M.C.: The local group: The ultimate deep field. Monthly Notices of the Royal Astronomical Society: Letters https://doi.org/10.1093/mnrasl/ slw121. http://mnrasl.oxfordjournals.org/content/early/2016/06/19/mnrasl.slw121.abstract (2016)

37. Brandt, T.D.: The Hipparcos-Gaia catalog of accelerations. ApJS 239(2), 31 (2018). https://doi.org/ 10.3847/1538-4365/aaec06, arXiv:1811.07283

38. Breivik, K., Chatterjee, S., Andrews, J.J.: Constraining compact object formation with 2M0521. ApJ 878(1), L4 (2019). https://doi.org/10.3847/2041-8213/ab21d3, arXiv:1810.08206

39. Brown, W.R.: Hypervelocity stars. ARA\&A 53, 15-49 (2015). https://doi.org/10.1146/annurevastro-082214-122230

40. Brunetti, M., Chiappini, C., Pfenniger, D.: Stellar diffusion in barred spiral galaxies. A\&A 534, A75 (2011). https://doi.org/10.1051/0004-6361/201117566, arXiv:1108.5631

41. Buder, S., Asplund, M., Duong, L., Kos, J., Lind, K., Ness, MK., Sharma, S., Bland-Hawthorn, J., Casey, AR., de Silva, GM., D’Orazi, V., Freeman, KC., Lewis, GF., Lin, J., Martell, SL., Schlesinger, KJ., Simpson, JD., Zucker, DB., Zwitter, T., Amarsi, AM., Anguiano, B., Carollo, D., Casagrande, L., Čotar, K., Cottrell, PL., da Costa, G., Gao, XD., Hayden, MR., Horner, J., Ireland, MJ., Kafle, PR., Munari, U., Nataf, DM., Nordlander, T., Stello, D., Ting, YS., Traven, G., Watson, F., Wittenmyer, RA., Wyse, RFG., Yong, D., Zinn, JC., Žerjal, M., Galah Collaboration.: The GALAH Survey: second data release. MNRAS 478(4), 4513-4552 (2018). https://doi.org/10.1093/mnras/sty1281, arXiv: 1804.06041

42. Buie, M., Porter, S.B., Verbiscer, A., Leiva, R., Keeney, B.A., Tsang, C., Baratoux, D., Skrutskie, M., Colas, F., Desmars, J., Stern, S.A., New Horizons MU69 Occultation Team: Pre-encounter update on (486958) 2014MU69 and occultation results from 2017 and 2018. In: AAS/Division for Planetary Sciences Meeting Abstracts \#50, AAS/Division for Planetary Sciences Meeting Abstracts, vol. 50, p. 509.06 (2018)

43. Caballero, J.A.: A review on substellar objects below the deuterium burning mass limit: Planets, Brown Dwarfs or What? Geosciences 8(10), 362 (2018). https://doi.org/10.3390/geosciences810 0362, arXiv: 1808.07798

44. Cantat-Gaudin, T., Jordi, C., Vallenari, A., Bragaglia, A., Balaguer-Núñez, L., Soubiran, C., Bossini, D., Moitinho, A., Castro-Ginard, A., Krone-Martins, A., Casamiquela, L., Sordo, R., Carrera, R.: A Gaia DR2 view of the open cluster population in the Milky Way. A\&A 618, A93 (2018). https://doi.org/10.1051/0004-6361/201833476, arXiv:1805.08726

45. Cantat-Gaudin, T., Krone-Martins, A., Sedaghat, N., Farahi, A., de Souza, R.S., Skalidis, R., Malz, A.I., Macêdo, S., Moews, B., Jordi, C., Moitinho, A., Castro-Ginard, A., Ishida, E.E.O., Heneka, C., Boucaud, A., Trindade, A.M.M.: Gaia DR2 unravels incompleteness of nearby cluster population: 
new open clusters in the direction of Perseus. A\&A 624, A126 (2019). https://doi.org/10.1051/00046361/201834453, arXiv: 1810.05494

46. Carlberg, R.G.: Dark matter sub-halo counts via star stream crossings. ApJ 748, 20 (2012). https://doi.org/10.1088/0004-637X/748/1/20, arXiv:1109.6022

47. Carpino, M., Milani, A., Chesley, S.R.: Error statistics of asteroid optical astrometric observations. Icarus 166, 248-270 (2003). https://doi.org/10.1016/S0019-1035(03)00051-4

48. Carr, B., Kühnel, F., Sandstad, M.: Primordial black holes as dark matter. Phys. Rev. D 94(8), 083504 (2016). https://doi.org/10.1103/PhysRevD.94.083504, arXiv:1607.06077

49. Carrasco, J.M., Catalán, S., Jordi, C., Tremblay, P.E., Napiwotzki, R., Luri, X., Robin, A.C., Kowalski, P.M.: Gaia photometry for white dwarfs. A\&A 565, A11 (2014). https://doi.org/10.1051/00046361/201220596, arXiv:1403.6045

50. Casamiquela, L., Blanco-Cuaresma, S., Carrera, R., Balaguer-Núñez, L., Jordi, C., Anders, F., Chiappini, C., Carbajo-Hijarrubia, J., Aguado, D.S., del Pino, A., Díaz-Pérez, L., Gallart, C., Pancino, E.: OCCASO - III. Iron peak and $\alpha$ elements of 18 open clusters. Comparison with chemical evolution models and field stars. MNRAS 490(2), 1821-1842 (2019). https://doi.org/10.1093/mnras/stz2595, arXiv: 1909.05865

51. Casertano, S., Lattanzi, M.G., Sozzetti, A., Spagna, A., Jancart, S., Morbidelli, R., Pannunzio, R., Pourbaix, D., Queloz, D.: Double-blind test program for astrometric planet detection with Gaia. A\&A 482, 699-729 (2008). https://doi.org/10.1051/0004-6361:20078997, arXiv:0802.0515

52. Castro-Ginard, A., Jordi, C., Luri, X., Julbe, F., Morvan, M., Balaguer-Núñez, L., Cantat-Gaudin, T.: A new method for unveiling open clusters in Gaia. New nearby open clusters confirmed by DR2. A\&A 618, A59 (2018). https://doi.org/10.1051/0004-6361/201833390, arXiv:1805.03045

53. Castro-Ginard, A., Jordi, C., Luri, X., Cantat-Gaudin, T., Balaguer-Núñez, L.: Hunting for open clusters in Gaia DR2: the Galactic anticentre. A\&A 627, A35 (2019). https://doi.org/10.1051/0004-6361/ 201935531, arXiv:1905.06161

54. Chemin, L., Renaud, F., Soubiran, C.: Incorrect rotation curve of the Milky Way. A\&A 578, A14 (2015). https://doi.org/10.1051/0004-6361/201526040, 1504.01507

55. Chesley, S.R., Baer, J., Monet, D.G.: Treatment of star catalog biases in asteroid astrometric observations. Icarus 210, 158-181 (2010). https://doi.org/10.1016/j.icarus.2010.06.003

56. Chiappini, C., Minchev, I., Starkenburg, E., Anders, F., Fusillo, N.G., Gerhard, O., Guiglion, G., Khalatyan, A., Kordopatis, G., Lemasle, B., Matijevic, G., Queiroz, A.B.D.A., Schwope, A., Steinmetz, M., Storm, J., Traven, G., Tremblay, P.E., Valentini, M., Andrae, R., Arentsen, A., Asplund, M., Bensby, T., Bergemann, M., Casagrande, L., Church, R., Cescutti, G., Feltzing, S., Fouesneau, M., Grebel, E.K., Kovalev, M., McMillan, P., Monari, G., Rybizki, J., Ryde, N., Rix, H.W., Walton, N., Xiang, M., Zucker, D., 4MIDABLE-Lr Team: 4MOST Consortium Survey 3: Milky Way Disc and Bulge Low-Resolution Survey (4MIDABLE-LR). Messenger 175, 30-34 (2019). https://doi.org/10.18727/0722-6691/5122, arXiv:1903.02469

57. Cirasuolo, M., Afonso, J., Carollo, M., Flores, H., Maiolino, R., Oliva, E., Paltani, S., Vanzi, L., Evans, C., Abreu, M., Atkinson, D., Babusiaux, C., Beard, S., Bauer, F., Bellazzini, M., Bender, R., Best, P., Bezawada, N., Bonifacio, P., Bragaglia, A., Bryson, I., Busher, D., Cabral, A., Caputi, K., Centrone, M., Chemla, F., Cimatti, A., Cioni, M.R., Clementini, G., Coelho, J., Crnojevic, D., Daddi, E., Dunlop, J., Eales, S., Feltzing, S., Ferguson, A., Fisher, M., Fontana, A., Fynbo, J., Garilli, B., Gilmore, G., Glauser, A., Guinouard, I., Hammer, F., Hastings, P., Hess, A., Ivison, R., Jagourel, P., Jarvis, M., Kaper, L., Kauffman, G., Kitching, A.T., Lawrence, A., Lee, D., Lemasle, B., Licausi, G., Lilly, S., Lorenzetti, D., Lunney, D., Maiolino, R., Mannucci, F., McLure, R., Minniti, D., Montgomery, D., Muschielok, B., Nandra, K., Navarro, R., Norberg, P., Oliver, S., Origlia, L., Padilla, N., Peacock, J., Pedichini, F., Peng, J., Pentericci, L., Pragt, J., Puech, M., Randich, S., Rees, P., Renzini, A., Ryde, N., Rodrigues, M., Roseboom, I., Royer, F., Saglia, R., Sanchez, A., Schiavon, R., Schnetler, H., Sobral, D., Speziali, R., Sun, D., Stuik, R., Taylor, A., Taylor, W., Todd, S., Tolstoy, E., Torres, M., Tosi, M., Vanzella, E., Venema, L., Vitali, F., Wegner, M., Wells, M., Wild, V., Wright, G., Zamorani, G., Zoccali, M.: MOONS: the Multi-Object Optical and Near-infrared Spectrograph for the VLT. In: Ground-based and Airborne Instrumentation for Astronomy V, Proc. SPIE, vol. 9147, p. 91470N (2014). https://doi.org/10.1117/12.2056012

58. Clarke, J.P., Wegg, C., Gerhard, O., Smith, L.C., Lucas, P.W., Wylie, S.M.: The Milky Way bar/bulge in proper motions: a 3D view from VIRAC \& Gaia. arXiv:1903.02003 (2019)

59. Cooray, A., Milosavljević, M.: What is $\mathrm{L}_{*}$ ? anatomy of the galaxy luminosity function. ApJ 627, L89-L92 (2005). https://doi.org/10.1086/432259, arXiv:astro-ph/0504580 
60. Dalton, G., Trager, S., Abrams, D.C., Bonifacio, P., López Aguerri, J.A., Middleton, K., Benn, C., Dee, K., Sayède, F., Lewis, I., Pragt, J., Pico, S., Walton, N., Rey, J., Allende Prieto, C., Peñate, J., Lhome, E., Agócs, T., Alonso, J., Terrett, D., Brock, M., Gilbert, J., Ridings, A., Guinouard, I., Verheijen, M., Tosh, I., Rogers, K., Steele, I., Stuik, R., Tromp, N., Jasko, A., Kragt, J., Lesman, D., Mottram, C., Bates, S., Gribbin, F., Rodriguez, L.F., Delgado, J.M., Martin, C., Cano, D., Navarro, R., Irwin, M., Lewis, J., Gonzalez Solares, E., O’Mahony, N., Bianco, A., Zurita, C., ter Horst, R., Molinari, E., Lodi, M., Guerra, J., Vallenari, A., Baruffolo, A.: Project overview and update on WEAVE: The next generation wide-field spectroscopy facility for the William Herschel Telescope. In: Ground-based and Airborne Instrumentation for Astronomy V, Proc. SPIE, vol. 9147, p. 91470L (2014). https://doi.org/10.1117/12.2055132, arXiv:1412.0843

61. Das, P., Sanders, J.L.: MADE: A spectroscopic mass, age, and distance estimator for red giant stars with Bayesian machine learning. MNRAS 484(1), 294-304 (2019). https://doi.org/10.1093/mnras/ sty2776, arXiv:1804.09596

62. de Jong, R.S., Bellido-Tirado, O., Chiappini, C., Depagne, É., Haynes, R., Johl, D., Schnurr, O., Schwope, A., Walcher, J., Dionies, F., Haynes, D., Kelz, A., Kitaura, F.S., Lamer, G., Minchev, I., Müller, V., Nuza, S.E., Olaya, J.C., Piffl, T., Popow, E., Steinmetz, M., Ural, U., Williams, M., Winkler, R., Wisotzki, L., Ansorge, W.R., Banerji, M., Gonzalez Solares, E., Irwin, M., Kennicutt, R.C., King, D., McMahon, R.G., Koposov, S., Parry, I.R., Sun, D., Walton, N.A., Finger, G., Iwert, O., Krumpe, M., Lizon, J.L., Vincenzo, M., Amans, J.P., Bonifacio, P., Cohen, M., Francois, P., Jagourel, P., Mignot, S.B., Royer, F., Sartoretti, P., Bender, R., Grupp, F., Hess, H.J., Lang-Bardl, F., Muschielok, B., Böhringer, H., Boller, T., Bongiorno, A., Brusa, M., Dwelly, T., Merloni, A., Nandra, K., Salvato, M., Pragt, J.H., Navarro, R., Gerlofsma, G., Roelfsema, R., Dalton, G.B., Middleton, K.F., Tosh, I.A., Boeche, C., Caffau, E., Christlieb, N., Grebel, E.K., Hansen, C., Koch, A., Ludwig, H.G., Quirrenbach, A., Sbordone, L., Seifert, W., Thimm, G., Trifonov, T., Helmi, A., Trager, S.C., Feltzing, S., Korn, A., Boland, W.: 4MOST: 4-metre multi-object spectroscopic telescope. In: Ground-based and Airborne Instrumentation for Astronomy IV, Proc. SPIE, vol. 8446, p. 84460T (2012). https://doi.org/10.1117/12.926239, arXiv:1206.6885

63. Debattista, V.P., Earp, S.W.F., Ness, M., Gonzalez, O.A.: Predicted stellar kinematics of a kiloparsecscale nuclear disc (or ring) in the Milky Way. Monthly Notices R. Astronom. Soc. 473(4), 52755285 (2017). https://doi.org/10.1093/mnras/stx2709. http://oup.prod.sis.lan/mnras/article-pdf/473/4/ 5275/21980804/stx2709.pdf

64. Dehnen, W., McLaughlin, D.E., Sachania, J.: The velocity dispersion and mass profile of the Milky Way. MNRAS 369, 1688-1692 (2006). https://doi.org/10.1111/j.1365-2966.2006.10404.x, arXiv:astro-ph/0603825

65. Delbó, M., Tanga, P., Mignard, F.: On the detection of the yarkovsky effect on near-earth asteroids by means of gaia. Planet Space Sci 56(14), 1823-1827 (2008). https://doi.org/10.1016/j.pss.2008. 02.029. http://www.sciencedirect.com/science/article/pii/S0032063308001839, mutual Events of the Uranian Satellites in 2007 - 2008Mutual Events of the Uranian Satellites in 2007-2008 and Further Observations in Network

66. DeMeo, F.E., Carry, B.: Solar System evolution from compositional mapping of the asteroid belt. Nature 505, 629-634 (2014). https://doi.org/10.1038/nature12908, arXiv:1408.2787

67. DeMeo, F.E., Binzel, R.P., Slivan, S.M., Bus, S.J.: An extension of the Bus asteroid taxonomy into the near-infrared. Icarus 202, 160-180 (2009). https://doi.org/10.1016/j.icarus.2009.02.005

68. Desmars, J., Bancelin, D., Hestroffer, D., Thuillot, W.: Statistical and numerical study of asteroid orbital uncertainty. A\&A 554, A32 (2013). https://doi.org/10.1051/0004-6361/201321090, arXiv: 1303.2946

69. Dias, W.S., Alessi, B.S., Moitinho, A., Lépine, J.RD.: New catalogue of optically visible open clusters and candidates. A\&A 389, 871-873 (2002). https://doi.org/10.1051/0004-6361:20020668, arXiv:astro-ph/0203351

70. Dominik, M., Sahu, K.C.: Astrometric microlensing of stars. ApJ 534(1), 213-226 (2000). https://doi.org/10.1086/308716

71. Dravins, D., Lindegren, L., Madsen, S.: Astrometric radial velocities. I. Non-spectroscopic methods for measuring stellar radial velocity. A\&A 348, 1040-1051 (1999). arXiv:astro-ph/9907145

72. Dressing, C.D., Charbonneau, D.: The occurrence of potentially habitable planets orbiting $\mathrm{m}$ dwarfs estimated from the full Kepler dataset and an empirical measurement of the detection sensitivity. ApJ 807, 45 (2015). https://doi.org/10.1088/0004-637X/807/1/45, arXiv:1501.01623

73. Eisenhauer, F., Perrin, G., Brandner, W., Straubmeier, C., Perraut, K., Amorim, A., Schöller, M., Gillessen, S., Kervella, P., Benisty, M., Araujo-Hauck, C., Jocou, L., Lima, J., Jakob, G., Haug, M., 
Clénet, Y., Henning, T., Eckart, A., Berger, J.P., Garcia, P., Abuter, R., Kellner, S., Paumard, T., Hippler, S., Fischer, S., Moulin, T., Villate, J., Avila, G., Gräter, A., Lacour, S., Huber, A., Wiest, M., Nolot, A., Carvas, P., Dorn, R., Pfuhl, O., Gendron, E., Kendrew, S., Yazici, S., Anton, S., Jung, Y., Thiel, M., Choquet, É., Klein, R., Teixeira, P., Gitton, P., Moch, D., Vincent, F., Kudryavtseva, N., Ströbele, S., Sturm, S., Fédou, P., Lenzen, R., Jolley, P., Kister, C., Lapeyrère, V., Naranjo, V., Lucuix, C., Hofmann, R., Chapron, F., Neumann, U., Mehrgan, L., Hans, O., Rousset, G., Ramos, J., Suarez, M., Lederer, R., Reess, J.M., Rohloff, R.R., Haguenauer, P., Bartko, H., Sevin, A., Wagner, K., Lizon, J.L., Rabien, S., Collin, C., Finger, G., Davies, R., Rouan, D., Wittkowski, M., Dodds-Eden, K., Ziegler, D., Cassaing, F., Bonnet, H., Casali, M., Genzel, R., Lena, P.: GRAVITY: Observing the universe in motion. Messenger 143, 16-24 (2011)

74. Ellis, R., Dawson, K., Bland-Hawthorn, J., Bacon, R., Bolton, A., Bremer, M., Brinchmann, J., Bundy, K., Conroy, C., Delabre, B., Dey, A., Drlica-Wagner, A., Greene, J., Guzzo, L., Johnson, J., Leauthaud, A., Lee, K.G., Pasquini, L., Pentericci, L., Richard, J., Rix, H.W., Rockosi, C., Schlegel, D., Slosar, A., Strauss, M., Takada, M., Tolstoy, E., Watson, D.: SpecTel: A 10-12 meter class spectroscopic survey telescope. arXiv:1907.06797 (2019)

75. Erkal, D., Belokurov, V., Laporte, C.F.P., Koposov, S.E., Li, T.S., Grillmair, C.J., Kallivayalil, N., Price-Whelan, A.M., Evans, N.W., Hawkins, K.: The total mass of the Large Magellanic Cloud from its perturbation on the Orphan stream. MNRAS 487(2), 2685-2700 (2019). https://doi.org/10.1093/mnras/stz1371, arXiv:1812.08192

76. Evans, D.F.: Evidence for Unresolved Exoplanet-hosting Binaries in Gaia DR2. Res. Notes Amer. Astronomic. Soc. 2(2), 20 (2018). https://doi.org/10.3847/2515-5172/aac173, arXiv:1804.11082

77. Evans, D.W., Riello, M., De Angeli, F., Carrasco, J.M., Montegriffo, P., Fabricius, C., Jordi, C., Palaversa, L., Diener, C., Busso, G., Cacciari, C., van Leeuwen, F., Burgess, P.W., Davidson, M., Harrison, D.L., Hodgkin, S.T., Pancino, E., Richards, P.J., Altavilla, G., Balaguer-Núñez, L., Barstow, M.A., Bellazzini, M., Brown, A.G.A., Castellani, M., Cocozza, G., De Luise, F., Delgado, A., Ducourant, C., Galleti, S., Gilmore, G., Giuffrida, G., Holl, B., Kewley, A., Koposov, S.E., Marinoni, S., Marrese, P.M., Osborne, P.J., Piersimoni, A., Portell, J., Pulone, L., Ragaini, S., Sanna, N., Terrett, D., Walton, N.A., Wevers, T., Wyrzykowski, Ł.: Gaia Data Release 2. Photometric content and validation. A\&A 616, A4 (2018). https://doi.org/10.1051/0004-6361/201832756, arXiv:1804.09368

78. Fanselow, J.L.: Observation Model and parameter partial for the JPL VLBI parameter Estimation Software MASTERFIT-v1.0. JPL Publ. 83- (1983)

79. Farnocchia, D., Chesley, S.R., Chamberlin, A.B., Tholen, D.J.: Star catalog position and proper motion corrections in asteroid astrometry. Icarus 245, 94-111 (2015). https://doi.org/10.1016/j. icarus.2014.07.033, arXiv:1407.8317

80. Feast, M.: Miras and other cool variables with GAIA. In: Munari, U. (ed.) GAIA Spectroscopy: Science and technology, astronomical society of the pacific conference series, vol. 298, p. 257 (2003). arXiv:astro-ph/0301101

81. Fischer, D.A., Anglada-Escude, G., Arriagada, P., Baluev, R.V., Bean, J.L., Bouchy, F., Buchhave, L.A., Carroll, T., Chakraborty, A., Crepp, J.R., Dawson, R.I., Diddams, S.A., Dumusque, X., Eastman, J.D., Endl, M., Figueira, P., Ford, E.B., Foreman-Mackey, D., Fournier, P., Fűrész, G., Gaudi, B.S., Gregory, P.C., Grundahl, F., Hatzes, A.P., Hébrard, G., Herrero, E., Hogg, D.W., Howard, A.W., Johnson, J.A., Jorden, P., Jurgenson, C.A., Latham, D.W., Laughlin, G., Loredo, T.J., Lovis, C., Mahadevan, S., McCracken, T.M., Pepe, F., Perez, M., Phillips, D.F., Plavchan, P.P., Prato, L., Quirrenbach, A., Reiners, A., Robertson, P., Santos, N.C., Sawyer, D., Segransan, D., Sozzetti, A., Steinmetz, T., Szentgyorgyi, A., Udry, S., Valenti, J.A., Wang, S.X., Wittenmyer, R.A., Wright, J.T.: State of the Field: Extreme Precision Radial Velocities. PASP 128(964), 066001 (2016). https://doi.org/10.1088/1538-3873/128/964/066001, arXiv:1602.07939

82. Folkner, W.M., Border, J.S.: Linking the planetary ephemeris to the International Celestial Reference Frame. Highlights Astronomy 16, 219-220 (2015). https://doi.org/10.1017/S1743921314005493

83. Friedli, D., Benz, W., Kennicutt, R.: On the influence of bars and star formation on galactic abundance gradients. ApJ 430, L105-L108 (1994). https://doi.org/10.1086/187449

84. Friske, J., Schönrich, R.: More than just a wrinkle: A wave-like pattern in radial velocity vs. angular momentum from Gaia Data. arXiv:1902.09569 (2019)

85. Frouard, J., Dorland, B.N., Makarov, V.V., Zacharias, N., Finch, C.T.: UrHip proper motion catalog. AJ 150, 141 (2015). https://doi.org/10.1088/0004-6256/150/5/141, arXiv:1509.05626

86. Fynbo, J.P.U., Krogager, J.K., Venemans, B., Noterdaeme, P., Vestergaard, M., Møller, P., Ledoux, C., Geier, S.: Optical/Near-infrared selection of red quasi-stellar objects: Evidence for Steep 
extinction curves toward galactic centers? ApJS 204(6) https://doi.org/10.1088/0067-0049/204/1/6, arXiv:1207.1193 (2013)

87. Gaia Collaboration, Prusti, T., de Bruijne, J.H.J., Brown, A.G.A., Vallenari, A., Babusiaux, C., Bailer-Jones, C.A.L., Bastian, U., Biermann, M., Evans, D.W.: The Gaia mission. A\&A 595, A1 (2016). https://doi.org/10.1051/0004-6361/201629272, arXiv:1609.04153

88. Gaia Collaboration, Katz, D., Antoja, T., Romero-Gómez, M., Drimmel, R., Reylé, C., Seabroke, G.M., Soubiran, C., Babusiaux, C., Di Matteo, P., Figueras, F., Poggio, E., Robin, A.C., Evans, D.W., Brown, A.G.A., Vallenari, A., Prusti, T., de Bruijne, J.H.J., Bailer-Jones, C.A.L., Biermann, M., Eyer, L., Jansen, F., Jordi, C., Klioner, S.A., Lammers, U., Lindegren, L., Luri, X., Mignard, F., Panem, C., Pourbaix, D., Randich, S., Sartoretti, P., Siddiqui, H.I., van Leeuwen, F., Walton, N.A., Arenou, F., Bastian, U., Cropper, M., Lattanzi, M.G., Bakker, J., Cacciari, C., Casta n, J., Chaoul, L., Cheek, N., De Angeli, F., Fabricius, C., Guerra, R., Holl, B., Masana, E., Messineo, R., Mowlavi, N., Nienartowicz, K., Panuzzo, P., Portell, J., Riello, M., Tanga, P., Thévenin, F., GraciaAbril, G., Comoretto, G., Garcia-Reinaldos, M., Teyssier, D., Altmann, M., Andrae, R., Audard, M., Bellas-Velidis, I., Benson, K., Berthier, J., Blomme, R., Burgess, P., Busso, G., Carry, B., Cellino, A., Clementini, G., Clotet, M., Creevey, O., Davidson, M., De Ridder, J., Delchambre, L., Dell'Oro, A., Ducourant, C., Fernández-Hernández, J., Fouesneau, M., Frémat, Y., Galluccio, L., García-Torres, M., González-Núñez, J., González-Vidal, J.J., Gosset, E., Guy, L.P., Halbwachs, J.L., Hambly, N.C., Harrison, D.L., Hernández, J., Hestroffer, D., Hodgkin, S.T., Hutton, A., Jasniewicz, G., Jean-Antoine-Piccolo, A., Jordan, S., Korn, A.J., Krone-Martins, A., Lanzafame, A.C., Lebzelter, T., Löffler, W., Manteiga, M., Marrese, P.M., Martín-Fleitas, J.M., Moitinho, A., Mora, A., Muinonen, K., Osinde, J., Pancino, E., Pauwels, T., Petit, J.M., Recio-Blanco, A., Richards, P.J., Rimoldini, L., Sarro, L.M., Siopis, C., Smith, M., Sozzetti, A., Süveges, M., Torra, J., van Reeven, W., Abbas, U., Abreu Aramburu, A., Accart, S., Aerts, C., Altavilla, G., Álvarez, M.A., Alvarez, R., Alves, J., Anderson, R.I., Andrei, A.H., Anglada Varela, E., Antiche, E., Arcay, B., Astraatmadja, T.L., Bach, N., Baker, S.G., Balaguer-Núñez, L., Balm, P., Barache, C., Barata, C., Barbato, D., Barblan, F., Barklem, P.S., Barrado, D., Barros, M., Barstow, M.A., Bartholomé Muñoz, L., Bassilana, J.L., Becciani, U., Bellazzini, M., Berihuete, A., Bertone, S., Bianchi, L., Bienaymé, O., BlancoCuaresma, S., Boch, T., Boeche, C., Bombrun, A., Borrachero, R., Bossini, D., Bouquillon, S., Bourda, G., Bragaglia, A., Bramante, L., Breddels, M.A., Bressan, A., Brouillet, N., Brüsemeister, T., Brugaletta, E., Bucciarelli, B., Burlacu, A., Busonero, D., Butkevich, A.G., Buzzi, R., Caffau, E., Cancelliere, R., Cannizzaro, G., Cantat-Gaudin, T., Carballo, R., Carlucci, T., Carrasco, J.M., Casamiquela, L., Castellani, M., Castro-Ginard, A., Charlot, P., Chemin, L., Chiavassa, A., Cocozza, G., Costigan, G., Cowell, S., Crifo, F., Crosta, M., Crowley, C., Cuypers, J., Dafonte, C., Damerdji, Y., Dapergolas, A., David, P., David, M., de Laverny, P., De Luise, F., De March, R., de Souza, R., de Torres, A., Debosscher, J., del Pozo, E., Delbó, M., Delgado, A., Delgado, H.E., Diakite, S., Diener, C., Distefano, E., Dolding, C., Drazinos, P., Durán, J., Edvardsson, B., Enke, H., Eriksson, K., Esquej, P., Eynard Bontemps, G., Fabre, C., Fabrizio, M., Faigler, S., Falc a, A.J., Farràs Casas, M., Federici, L., Fedorets, G., Fernique, P., Filippi, F., Findeisen, K., Fonti, A., Fraile, E., Fraser, M., Frézouls, B, Gai, M., Galleti, S., Garabato, D., García-Sedano, F, Garofalo, A., Garralda, N., Gavel, A., Gavras, P., Gerssen, J., Geyer, R., Giacobbe, P., Gilmore, G., Girona, S., Giuffrida, G., Glass, F., Gomes, M., Granvik, M., Gueguen, A., Guerrier, A., Guiraud, J., Gutié, R, Haigron, R., Hatzidimitriou, D., Hauser, M., Haywood, M., Heiter, U., Helmi, A., Heu, J., Hilger, T., Hobbs, D., Hofmann, W., Holland, G., Huckle, H.E., Hypki, A., Icardi, V., Janßen, K, Jevardat de Fombelle, G., Jonker, P.G., Juhász, ÁL, Julbe, F., Karampelas, A., Kewley, A., Klar, J., Kochoska, A., Kohley, R., Kolenberg, K., Kontizas, M., Kontizas, E., Koposov, S.E., Kordopatis, G., Kostrzewa-Rutkowska, Z., Koubsky, P., Lambert, S., Lanza, A.F., Lasne, Y., Lavigne, J.B., Le Fustec, Y., Le Poncin-Lafitte, C., Lebreton, Y., Leccia, S., Leclerc, N., Lecoeur-Taibi, I., Lenhardt, H., Leroux, F., Liao, S. Licata, E., Lindstrøm, HEP, Lister, T.A., Livanou, E., Lobel, A., López, M, Managau, S., Mann, R.G., Mantelet, G., Marchal, O., Marchant, J.M., Marconi, M., Marinoni, S., Marschalkó, G, Marshall, D.J., Martino, M., Marton, G., Mary, N., Massari, D., Matijevič, G, Mazeh, T., McMillan, P.J., Messina, S., Michalik, D., Millar, N.R., Molina, D., Molinaro, R., Molnár, L, Montegriffo, P., Mor, R., Morbidelli, R., Morel, T., Morris, D., Mulone, A.F., Muraveva, T., Musella, I., Nelemans, G., Nicastro, L., Noval, L., O’Mullane, W., Ordénovic, C, Ordóñez-Blanco, D, Osborne, P., Pagani, C., Pagano, I., Pailler, F.: Gaia Data Release 2. Mapping the Milky Way disc kinematics. A\&A 616, A11 (2018a). https://doi.org/10.1051/0004-6361/201832865, arXiv:1804.09380 
89. Gaia Collaboration, Mignard, F., Klioner, S.A., Lindegren, L., Hernández, J., Bastian, U., Bombrun, A., Hobbs, D., Lammers, U., Michalik, D.: Gaia Data Release 2. The celestial reference frame (GaiaCRF2). A\&A 616, A14 (2018b). https://doi.org/10.1051/0004-6361/201832916

90. Garavito-Camargo, N., Besla, G., Laporte, C.F.P., Johnston, K.V., Gómez, F.A., Watkins, L.L.: Hunting for the dark matter wake induced by the large magellanic cloud. arXiv:1902.05089 (2019)

91. García-Berro, E., Oswalt, T.D.: The white dwarf luminosity function. New A Rev. 72, 1-22 (2016). https://doi.org/10.1016/j.newar.2016.08.001, arXiv:1608.02631

92. Gentile Fusillo, N.P., Tremblay, P.E., Gänsicke, B.T., Manser, C.J., Cunningham, T., Cukanovaite, E., Hollands, M., Marsh, T., Raddi, R., Jordan, S., Toonen, S., Geier, S., Barstow, M., Cummings, J.D.: A Gaia Data Release 2 catalogue of white dwarfs and a comparison with SDSS. MNRAS 482, 4570-4591 (2019). https://doi.org/10.1093/mnras/sty3016, arXiv:1807.03315

93. Gilmore, G.: The star formation history of the Milky Way. In: Funes, J.G., Corsini, E.M. (eds.) Galaxy Disks and Disk Galaxies, Astronomical Society of the Pacific Conference Series, vol. 230, pp. 3-12 (2001). arXiv:astro-ph/0011450

94. Girardi, L., Groenewegen, M.A.T., Hatziminaoglou, E., da Costa, L.: Star counts in the Galaxy. Simulating from very deep to very shallow photometric surveys with the TRILEGAL code. A\&A 436, 895-915 (2005). https://doi.org/10.1051/0004-6361:20042352, arXiv:astro-ph/0504047

95. Girardi, L., Barbieri, M., Groenewegen, M.A.T., Marigo, P., Bressan, A., Rocha-Pinto, H.J., Santiago, B.X., Camargo, J.I.B., da Costa, L.N.: TRILEGAL, a TRIdimensional modeL of thE GALaxy: Status and Future. Astrophys. Space Sci. Proc. 26, 165 (2012). https://doi.org/10.1007/978-3-642-18418-5_17

96. Gnedin, O.Y., Gould, A., Miralda-Escudé, J., Zentner, A.R.: Probing the Shape of the Galactic Halo with Hypervelocity Stars. ApJ 634, 344-350 (2005). https://doi.org/10.1086/496958, arXiv:astro-ph/0506739

97. Gonzalez, O.A., Battaglia, G.: From the inner Milky Way to Local Volume galaxies: resolved stellar populations with ELT-HARMONI. arXiv:1810.04422 (2018)

98. Governato, F., Zolotov, A., Pontzen, A., Christensen, C., Oh, S.H., Brooks, A.M., Quinn, T., Shen, S., Wadsley, J.: Cuspy no more: how outflows affect the central dark matter and baryon distribution in $\Lambda$ cold dark matter galaxies. MNRAS 422, 1231-1240 (2012). https://doi.org/10.1111/j.1365-2966. 2012.20696.X, arXiv:1202.0554

99. Grady, J., Belokurov, V., Evans, N.W.: Age gradients throughout the Galaxy with long-period variables. MNRAS 483, 3022-3035 (2019). https://doi.org/10.1093/mnras/sty3284, arXiv:1804.09186

100. Grand, R.J.J., Springel, V., Kawata, D., Minchev, I., Sánchez-Blázquez, P., Gómez, F.A., Marinacci, F., Pakmor, R., Campbell, D.J.R.: Spiral-induced velocity and metallicity patterns in a cosmological zoom simulation of a Milky Way-sized galaxy. MNRAS 460(1), L94-L98 (2016). https://doi.org/10.1093/mnrasl/slw086, arXiv:1604.01027

101. Gwinn, C.R., Eubanks, T.M., Pyne, T., Birkinshaw, M., Matsakis, D.N.: Quasar proper motions and low-frequency gravitational waves. ApJ 485, 87-91 (1997). arXiv:astro-ph/9610086

102. Han, C., Jung, Y.K., Udalski, A., Sumi, T., Gaudi, B.S., Gould, A., Bennett, D.P., Tsapras, Y., Szymański, M.K., Kubiak, M., Pietrzyński, G., Soszyński, I., Skowron, J., Kozłowski, S., Poleski, R., Ulaczyk, K., Wyrzykowski, Ł., Pietrukowicz, P., O.G.L.E. Collaboration, Abe, F., Bond, I.A., Botzler, C.S., Chote, P., Freeman, M., Fukui, A., Furusawa, K., Harris, P., Itow, Y., Ling, C.H., Masuda, K., Matsubara, Y., Muraki, Y., Ohnishi, K., Rattenbury, N.J., Saito, T., Sullivan, D.J., Sweatman, W.L., Suzuki, D., Tristram, P.J., Wada, K., Yock, P.C.M., M.O.A. Collaboration, Batista, V., Christie, G., Choi, J.Y., DePoy, D.L., Dong, S., Hwang, K.H., Kavka, A., Lee, C.U., Monard, L.A.G., Natusch, T., Ngan, H., Park, H., Pogge, R.W., Porritt, I., Shin, I.G., Tan, T.G., Yee, J.C., $\mu$ FUN Collaboration, Alsubai, K.A., Bozza, V., Bramich, D.M., Browne, P., Dominik, M., Horne, K., Hundertmark, M., Ipatov, S., Kains, N., Liebig, C., Snodgrass, C., Steele, I.A., Street, R.A., RoboNet Collaboration: Microlensing discovery of a tight, low-mass-ratio planetary-mass object around an old field brown dwarf. ApJ 778(1), 38 (2013). https://doi.org/10.1088/0004-637X/778/1/38, arXiv:1307.6335

103. Heintz, K.E., Fynbo, J.P.U., Høg, E.: A study of purely astrometric selection of extragalactic point sources with Gaia. A\&A 578, A91 (2015). https://doi.org/10.1051/0004-6361/201526038, arXiv: 1503.02874

104. Heintz, K.E., Fynbo, J.P.U., Høg, E., Møller, P., Krogager, J.K., Geier, S., Jakobsson, P., Christensen, L.: Unidentified quasars among stationary objects from Gaia DR2. A\&A 615, L8 (2018). https://doi.org/10.1051/0004-6361/201833396, arXiv:1805.03394 
105. Helmi, A., Babusiaux, C., Koppelman, H.H., Massari, D., Veljanoski, J., Brown, A.G.A.: The merger that led to the formation of the Milky Way's inner stellar halo and thick disk. Nature 563(7729), 85-88 (2018). https://doi.org/10.1038/s41586-018-0625-x, arXiv:1806.06038

106. Hilton, J.L., Seidelmann, P.K., Middour, J.: Prospects for determining asteroid masses. AJ 112, 2319 (1996). https://doi.org/10.1086/118185

107. Hinshaw, G., Weiland, J.L., Hill, R.S., Odegard, N., Larson, D., Bennett, C.L., Dunkley, J., Gold, B., Greason, M.R., Jarosik, N., Komatsu, E., Nolta, M.R., Page, L., Spergel, D.N., Wollack, E., Halpern, M., Kogut, A., Limon, M., Meyer, S.S., Tucker, G.S., Wright, E.L.: Five-Year Wilkinson Microwave Anisotropy Probe Observations: Data Processing, Sky Maps, and Basic Results. ApJS 180, 225-245 (2009). https://doi.org/10.1088/0067-0049/180/2/225, arXiv:0803.0732

108. Hobbs, D., Høg, E., Mora, A., Crowley, C., McMillan, P., Ranalli, P., Heiter, U., Jordi, C., Hambly, N., Church, R., Anthony, B., Tanga, P., Chemin, L., Portail, J., Jiménez-Esteban, F., Klioner, S., Mignard, F., Fynbo, J., Wyrzykowski, Ł., Rybicki, K., Anderson, R.I., Cellino, A., Fabricius, C., Davidson, M., Lindegren, L.: GaiaNIR: Combining optical and Near-Infra-Red (NIR) capabilities with Time-Delay-Integration (TDI) sensors for a future Gaia-like mission. arXiv:1609.07325 (2016)

109. Hobbs, D., Leitz, C., Bartlett, J., Hepburn, I., Kawata, D., Cropper, M., Mazin, B., Brown, A., Makarov, V., McArthur, B.: Astro2020 Activity, Project of State of the Profession Consideration (APC) White Paper: All-Sky Near Infrared Space Astrometry. State of the Profession Considerations: Development of Scanning NIR Detectors for Astronomy. arXiv:1907.05191 (2019)

110. Høg, E.: Absolute astrometry in the next 50 years. arXiv:1408.2190 (2014)

111. Høg, E., Kaplan, G.: Solar system and small-field astrometry. arXiv:1408.3302 (2014)

112. Holley-Bockelmann, K., Weinberg, M., Katz, N.: Bar-induced evolution of dark matter cusps. MNRAS 363, 991-1007 (2005). https://doi.org/10.1111/j.1365-2966.2005.09501.x, arXiv:astro-ph/0306374

113. Hunt, J.A.S., Kawata, D.: M2M modelling of the Galactic disc via PRIMAL: fitting to Gaia error added data. MNRAS 443(3), 2112-2125 (2014). https://doi.org/10.1093/mnras/stu1306, arXiv: 1312.4949

114. Isern, J., García-Berro, E., Salaris, M.: What are white dwarfs telling us about the galactic disk and halo?. In: von Hippel, T., Simpson, C., Manset, N. (eds.) Astrophysical Ages and Times Scales, vol. 245, p. 328 (2001)

115. Ivezić, Z., Monet, D.G., Claver, C.F., Axelrod, T.S., Gizis, J., Lupton, R.: LSST Astrometry: Simulations and numerical studies. In: American Astronomical Society Meeting Abstracts, vol. 221, p. 247.06 (2013)

116. Ivezić, Ž., Kahn, S.M., Tyson, J.A., Abel, B., Acosta, E., Allsman, R., Alonso, D., AlSayyad, Y., Anderson, S.F., Andrew, J., et al: LSST: From science drivers to reference design and anticipated data products. ApJ 873, 111 (2019). https://doi.org/10.3847/1538-4357/ab042c, arXiv:0805.2366

117. Jiménez-Esteban, F.M., Engels, D.: Study of extremely reddened AGB stars in the Galactic bulge. A\&A 579, A76 (2015). https://doi.org/10.1051/0004-6361/201424609, arXiv:1504.05712

118. Jiménez-Esteban, F.M., García-Lario, P., Engels, D., Manchado, A.: Near-IR variability properties of a selected sample of AGB stars. A\&A 458, 533-539 (2006). https://doi.org/10.1051/0004-6361:20053982, arXiv:astro-ph/0606380

119. Jiménez-Esteban, F.M., Torres, S., Rebassa-Mansergas, A., Skorobogatov, G., Solano, E., Cantero, C., Rodrigo, C.: A white dwarf catalogue from Gaia-DR2 and the Virtual Observatory. MNRAS 480, 4505-4518 (2018). https://doi.org/10.1093/mnras/sty2120, arXiv:1807.02559

120. Johnston, K.V., Price-Whelan, A.M., Bergemann, M., Laporte, C., Li, T.S., Sheffield, A.A., Majewski, S.R., Beaton, R.S., Sesar, B., Sharma, S.: Disk Heating, Galactoseismology, and the Formation of Stellar Halos. Galaxies 5(3), 44 (2017). https://doi.org/10.3390/galaxies5030044, arXiv:1709.00491

121. Kamdar, H., Conroy, C., Ting, Y.S., Bonaca, A., Johnson, B., Cargile, P.: A dynamical model for clustered star formation in the galactic disk. arXiv:1902.10719 (2019a)

122. Kamdar, H., Conroy, C., Ting, Y.S., Bonaca, A., Smith, M., Brown, A.G.A.: Stars that Move Together Were Born Together. arXiv:1904.02159 (2019b)

123. Kardashev, N.S.: Cosmological proper motion. AZh 63, 845-849 (1986)

124. Kawata, D., Baba, J., Ciucă, I., Cropper, M., Grand, R.J.J., Hunt, J.A.S., Seabroke, G.: Radial distribution of stellar motions in Gaia DR2. MNRAS 479(1), L108-L112 (2018). https://doi.org/10.1093/mnrasl/sly107, arXiv:1804.10175 
125. Kawata, D., Bovy, J., Matsunaga, N., Baba, J.: Galactic rotation from Cepheids with Gaia DR2 and effects of non-axisymmetry. MNRAS 482(1), 40-51 (2019). https://doi.org/10.1093/mnras/sty2623, arXiv: 1803.05927

126. Kerins, E., Robin, A.C., Marshall, D.J.: Synthetic microlensing maps of the Galactic bulge. MNRAS 396, 1202-1210 (2009). https://doi.org/10.1111/j.1365-2966.2009.14791.x, arXiv:0805.4626

127. Kervella, P., Arenou, F., Mignard, F., Thévenin, F.: Stellar and substellar companions of nearby stars from Gaia DR2. Binarity from proper motion anomaly. A\&A 623, A72 (2019). https://doi.org/10.1051/0004-6361/201834371, arXiv:1811.08902

128. Kharchenko, N.V., Piskunov, A.E., Schilbach, E., Röser, S., Scholz, R.D.: Global survey of star clusters in the Milky Way. II. The catalogue of basic parameters. A\&A 558, A53 (2013). https://doi.org/10.1051/0004-6361/201322302, arXiv:1308.5822

129. Kirkpatrick, J.D., Cruz, K.L., Barman, T.S., Burgasser, A.J., Looper, D.L., Tinney, C.G., Gelino, C.R., Lowrance, P.J., Liebert, J., Carpenter, J.M., Hillenbrand, L.A., Stauffer, J.R.: A Sample of Very Young Field L Dwarfs and Implications for the Brown Dwarf "Lithium Test" at Early Ages. ApJ 689(2), 1295-1326 (2008). https://doi.org/10.1086/592768, arXiv:0808.3153

130. Kirkpatrick, J.D., Martin, E.C., Smart, R.L., Cayago, A.J., Beichman, C.A., Marocco, F., Gelino, C.R., Faherty, J.K., Cushing, M.C., Schneider, A.C., Mace, G.N., Tinney, C.G., Wright, E.L., Lowrance, P.J., Ingalls, J.G., Vrba, F.J., Munn, J.A., Dahm, S.E., McLean, I.S.: Preliminary Trigonometric Parallaxes of 184 Late-T and Y Dwarfs and an Analysis of the Field Substellar Mass Function into the "Planetary" Mass Regime. ApJS 240(2), 19 (2019). https://doi.org/10.3847/1538-4365/aaf 6af, arXiv: 1812.01208

131. Klioner, S.A.: A practical relativistic model for microarcsecond astrometry in space. AJ 125, 15801597 (2003). https://doi.org/10.1086/367593

132. Klioner, S.A.: Gaia-like astrometry and gravitational waves. Classic. Quantum Grav. 35(4), 045005 (2018). https://doi.org/10.1088/1361-6382/aa9f57, arXiv:1710.11474

133. Kollmeier, J.A., Zasowski, G., Rix, H.W., Johns, M., Anderson, S.F., Drory, N., Johnson, J.A., Pogge, R.W., Bird, J.C., Blanc, G.A.: SDSS-V: Pioneering Panoptic Spectroscopy. arXiv:1711.03234 (2017)

134. Kopeikin, S.M., Makarov, V.V.: Astrometric effects of secular aberration. AJ 131, 1471-1478 (2006). https://doi.org/10.1086/500170, arXiv:astro-ph/0508505

135. Korol, V., Rossi, E.M.: Synergies between Gaia and LISA missions for Galactic multi-messenger studies. In: ESLAB 53: TheGaiaUnivers, Zenodo https://doi.org/10.5281/zenodo.3237213 (2019)

136. Korol, V., Rossi, E.M., Barausse, E.: A multimessenger study of the Milky Way's stellar disc and bulge with LISA, Gaia, and LSST. Monthly Notices R. Astronomic. Soc. 483(4), 55185533 (2018a). https://doi.org/10.1093/mnras/sty3440, http://oup.prod.sis.lan/mnras/article-pdf/483/ 4/5518/27496911/sty3440.pdf

137. Korol, V., Rossi, E.M., Barausse, E.: Constraining the Milky Way potential with Double White Dwarfs. arXiv:1810.03938 (2018b)

138. Kovalevsky, J.: Aberration in proper motions. A\&A 404, 743-747 (2003). https://doi.org/10.1051/ 0004-6361:20030560

139. Kristian, J., Sachs, R.K.: Observations in cosmology. ApJ 143, 379 (1966). https://doi.org/10.1086/1 48522

140. Krogager, J.K., Fynbo, J.P.U., Heintz, K.E., Geier, S., Ledoux, C., Møller, P., Noterdaeme, P., Venemans, B.P., Vestergaard, M.: The Extended High A(V) Quasar Survey: Searching for Dusty Absorbers toward Mid-infrared-selected Quasars. ApJ 832, 49 (2016). https://doi.org/10.3847/0004637X/832/1/49, arXiv:1608.08404

141. Kuchynka, P.: A new approach to asteroid modeling in a planetary ephemeris. In: IAU Joint Discussion, IAU Joint Discussion, vol. 7, p. 38 (2012)

142. Kuhn, M.A., Feigelson, E.D., Getman, K.V., Sills, A., Bate, M.R., Borissova, J.: The spatial structure of young stellar clusters. III. Physical properties and evolutionary states. ApJS 812, 131 (2015). https://doi.org/10.1088/0004-637X/812/2/131, arXiv:1507.05653

143. Liebert, J., Bergeron, P., Holberg, J.B.: The formation rate and mass and luminosity functions of da white dwarfs from the Palomar green survey. ApJS 156, 47-68 (2005). https://doi.org/10.1086/425 738, arXiv:astro-ph/0406657

144. Lin, C.C., Shu, F.H.: On the spiral structure of disk galaxies. ApJ 140, 646 (1964). https://doi.org/10. $1086 / 147955$ 
145. Lindegren, L., Lammers, U., Hobbs, D., O’Mullane, W., Bastian, U., Hernández, J.: The astrometric core solution for the Gaia mission. Overview of models, algorithms, and software implementation. A\&A 538, A78 (2012). https://doi.org/10.1051/0004-6361/201117905, arXiv:1112.4139

146. Lindegren, L., Hernández, J., Bombrun, A., Klioner, S., Bastian, U., Ramos-Lerate, M., de Torres, A., Steidelmüller, H., Stephenson, C., Hobbs, D.: Gaia Data Release 2. The astrometric solution. A\&A 616, A2 (2018). https://doi.org/10.1051/0004-6361/201832727, arXiv:1804.09366

147. Magrin, D., Ragazzoni, R., Rauer, H., Pagano, I., Nascimbeni, V., Piotto, G., Viotto, V., Piazza, D., Bandy, T., Basso, S.: PLATO: the ESA mission for exo-planets discovery. In: Proc. SPIE, Society of Photo-Optical Instrumentation Engineers (SPIE) Conference Series, vol. 10698, p. 106984X (2018). https://doi.org/10.1117/12.2313538

148. Majaess, D., Dékány, I., Hajdu, G., Minniti, D., Turner, D., Gieren, W.: Establishing the Galactic Centre distance using VVV Bulge RR Lyrae variables. Ap\&SS 363(6), 127 (2018). https://doi.org/10.1007/s10509-018-3346-6, arXiv:1805.04119

149. Majewski, S.R., Schiavon, R.P., Frinchaboy, P.M., Allende Prieto, C., Barkhouser, R., Bizyaev, D., Blank, B., Brunner, S., Burton, A., Carrera, R., Chojnowski, S.D., Cunha, K., Epstein, C., Fitzgerald, G., Garcia Perez, A.E., Hearty, F.R., Henderson, C., Holtzman, J.A., Johnson, J.A., Lam, C.R., Lawler, J.E., Maseman, P., Meszaros, S., Nelson, M., Coung Nguyen, D., Nidever, D.L., Pinsonneault, M., Shetrone, M., Smee, S., Smith, V.V., Stolberg, T., Skrutskie, M.F., Walker, E., Wilson, J.C., Zasowski, G., Anders, F., Basu, S., Beland, S., Blanton, M.R., Bovy, J., Brownstein, J.R., Carlberg, J., Chaplin, W., Chiappini, C., Eisenstein, D.J., Elsworth, Y., Feuillet, D., Fleming, S.W., Galbraith-Frew, J., Garcia, R.A., Anibal Garcia-Hernandez, D., Gillespie, B.A., Girardi, L., Gunn, J.E., Hasselquist, S., Hayden, M.R., Hekker, S., Ivans, I., Kinemuchi, K., Klaene, M., Mahadevan, S., Mathur, S., Mosser, B., Muna, D., Munn, J.A., Nichol, R.C., O’Connell, R.W., Robin, A.C., Rocha-Pinto, H., Schultheis, M., Serenelli, A.M., Shane, N., Silva Aguirre, V., Sobeck, J.S., Thompson, B., Troup, N.W., Weinberg, D.H., Zamora, O.: The apache point observatory galactic evolution experiment (APOGEE). Astronom J 154(3), 94 (2017). https://doi.org/10.3847/1538-3881/aa784d

150. Makarov, V.V., Berghea, C.T., Frouard, J., Fey, A., Schmitt, H.R.: The Precious Set of Radiooptical Reference Frame Objects in the Light of Gaia DR2 Data. ApJ 873, 132 (2019). https://doi.org/10.3847/1538-4357/aafa1c, arXiv:1811.10117

151. Malhan, K., Ibata, R.A.: Constraining the Milky Way halo potential with the GD-1 stellar stream. MNRAS 486(3), 2995-3005 (2019). https://doi.org/10.1093/mnras/stz1035, arXiv:1807.05994

152. Malhan, K., Ibata, R.A., Martin, N.F.: Ghostly tributaries to the Milky Way: charting the halo's stellar streams with the Gaia DR2 catalogue. MNRAS 481(3), 3442-3455 (2018). https://doi.org/10.1093/mnras/sty2474, arXiv:1804.11339

153. Malkin, Z.: On the implications of the Galactic aberration in proper motions for the Celestial Reference Frame. MNRAS 445, 845-849 (2014). https://doi.org/10.1093/mnras/stu1796, arXiv:1409.0504

154. McArthur, B., Hobbs, D., Høg, E., Makarov, V., Sozzetti, A., Brown, A., Krone Martins, A., Bartlett, J.L., Tomsick, J., Shao, M., Benedict, F., Bendek, E., Boehm, C., Conroy, C., Uldall Fynbo, J.P., Gnedin, O., Hillenbrand, L., Lindegren, L., Rodriguez, D.R., White, R., Turyshev, S., Unwin, S., Zhai, C.: All-Sky Near Infrared Space Astrometry. arXiv:1904.08836 (2019)

155. McArthur, B.E., Benedict, G.F., Barnes, R., Martioli, E., Korzennik, S., Nelan, E., Butler, R.P.: New Observational Constraints on the $v$ Andromedae System with Data from the Hubble Space Telescope and Hobby-Eberly Telescope. ApJ 715, 1203-1220 (2010). https://doi.org/10.1088/0004-637X/715/ 2/1203

156. McKee, C.F., Parravano, A., Hollenbach, D.J.: Stars, gas, and dark matter in the solar neighborhood. ApJ 814(1), 13 (2015). https://doi.org/10.1088/0004-637X/814/1/13, arXiv:1509.05334

157. McMillan, P.J., Kordopatis, G., Kunder, A., Binney, J., Wojno, J., Zwitter, T., Steinmetz, M., BlandHawthorn, J., Gibson, B.K., Gilmore, G., Grebel, E.K., Helmi, A., Munari, U., Navarro, J.F., Parker, Q.A., Seabroke, G., Watson, F., Wyse, R.F.G.: Improved distances and ages for stars common to TGAS and RAVE. Monthly Notices R. Astronomic. Soc. 477(4), 5279-5300 (2018). https://doi.org/ 10.1093/mnras/sty990, http://oup.prod.sis.lan/mnras/article-pdf/477/4/5279/25020659/sty990.pdf

158. Meidt, S.E., Rand, R.J., Merrifield, M.R.: Uncovering the origins of spiral structure by measuring radial variation in pattern speeds. ApJ 702(1), 277-290 (2009). https://doi.org/10.1088/0004-637X/ 702/1/277, arXiv:0907.3443

159. Michalik, D., Lindegren, L., Hobbs, D., Lammers, U.: Joint astrometric solution of HIPPARCOS and Gaia. A recipe for the Hundred Thousand Proper Motions project. A\&A 571, A85 (2014). https://doi.org/10.1051/0004-6361/201424606, arXiv:1407.4025 
160. Michalik, D., Lindegren, L., Hobbs, D.: The Tycho-Gaia astrometric solution. How to get 2.5 million parallaxes with less than one year of Gaia data. A\&A 574, A115 (2015). https://doi.org/10.1051/ 0004-6361/201425310, arXiv:1412.8770

161. Miglio, A., Chiappini, C., Mosser, B., Davies, G.R., Freeman, K., Girardi, L., Jofré, P., Kawata, D., Rendle, B.M., Valentini, M., Casagrande, L., Chaplin, W.J., Gilmore, G., Hawkins, K., Holl, B., Appourchaux, T., Belkacem, K., Bossini, D., Brogaard, K., Goupil, M.J., Montalbán, J., Noels, A., Anders, F., Rodrigues, T., Piotto, G., Pollacco, D., Rauer, H., Allende Prieto, C., Avelino, P.P., Babusiaux, C., Barban, C., Barbuy, B., Basu, S., Baudin, F., Benomar, O., Bienaymé, O., Binney, J., Bland-Hawthorn, J., Bressan, A., Cacciari, C., Campante, T.L., Cassisi, S., ChristensenDalsgaard, J., Combes, F., Creevey, O., Cunha, M.S., Jong, R.S., Laverny, P., Degl'Innocenti, S., Deheuvels, S., Depagne, É., Ridder, J., Di Matteo, P., Di Mauro, M.P., Dupret, M.A., Eggenberger, P., Elsworth, Y., Famaey, B., Feltzing, S., García, R.A., Gerhard, O., Gibson, B.K., Gizon, L., Haywood, M., Handberg, R., Heiter, U., Hekker, S., Huber, D., Ibata, R., Katz, D., Kawaler, S.D., Kjeldsen, H., Kurtz, D.W., Lagarde, N., Lebreton, Y., Lund, M.N., Majewski, S.R., Marigo, P., Martig, M., Mathur, S., Minchev, I., Morel, T., Ortolani, S., Pinsonneault, M.H., Plez, B., Prada Moroni, P.G., Pricopi, D., Recio-Blanco, A., Reylé, C., Robin, A., Roxburgh, I.W., Salaris, M., Santiago, B.X., Schiavon, R., Serenelli, A., Sharma, S., Silva Aguirre, V., Soubiran, C., Steinmetz, M., Stello, D., Strassmeier, K.G., Ventura, P., Ventura, R., Walton, N.A., Worley, C.C.: PLATO as it is : A legacy mission for Galactic archaeology. Astronomische Nachrichten 338, 644-661 (2017). https://doi.org/10.1002/asna.201713385, arXiv:1706.03778

162. Mignard, F., Klioner, S.: Analysis of astrometric catalogues with vector spherical harmonics. A\&A 547, A59 (2012). https://doi.org/10.1051/0004-6361/201219927, arXiv:1207.0025

163. Minchev, I., Famaey, B.: A new mechanism for radial migration in galactic disks: SpiralBar resonance overlap. ApJ 722, 112-121 (2010). https://doi.org/10.1088/0004-637X/722/1/112, arXiv:0911.1794

164. Minchev, I., Chiappini, C., Martig, M.: Chemodynamical evolution of the Milky Way disk. I. The solar vicinity. A\&A 558, A9 (2013). https://doi.org/10.1051/0004-6361/201220189, arXiv: 1208.1506

165. Minchev, I., Chiappini, C., Martig, M.: Chemodynamical evolution of the Milky Way disk. II. Variations with Galactic radius and height above the disk plane. A\&A 572, A92 (2014a). https://doi.org/10.1051/0004-6361/201423487, arXiv:1401.5796

166. Minchev, I., Chiappini, C., Martig, M., Steinmetz, M., de Jong, R.S., Boeche, C., Scannapieco, C., Zwitter, T., Wyse, R.F.G., Binney, J.J., Bland-Hawthorn, J., Bienaymé, O., Famaey, B., Freeman, K.C., Gibson, B.K., Grebel, E.K., Gilmore, G., Helmi, A., Kordopatis, G., Lee, Y.S., Munari, U., Navarro, J.F., Parker, Q.A., Quillen, A.C., Reid, W.A., Siebert, A., Siviero, A., Seabroke, G., Watson, F., Williams, M.: A New Stellar Chemo-Kinematic relation reveals the merger history of the Milky Way disk. ApJ 781(1), L20 (2014b). https://doi.org/10.1088/2041-8205/781/1/L20, arXiv:1310.5145

167. Minniti, D., Lucas, P.W., Emerson, J.P., Saito, R.K., Hempel, M., Pietrukowicz, P., Ahumada, A.V., Alonso, M.V., Alonso-Garcia, J., Arias, J.I.: VISTA Variables in the Via Lactea (VVV): The public ESO near-IR variability survey of the Milky Way. New A 15(5), 433-443 (2010). https://doi.org/10.1016/j.newast.2009.12.002, arXiv:0912.1056

168. Miyachi, Y., Sakai, N., Kawata, D., Baba, J., Honma, M., Matsunaga, N., Fujisawa, K.: Stellar Overdensity in the Local Arm in Gaia DR2. arXiv:1907.03763 (2019)

169. Mor, R., Robin, A.C., Figueras, F., Roca-Fàbrega, S., Luri, X.: Gaia DR2 reveals a star formation burst in the disc 2-3 Gyr ago. A\&A 624, L1 (2019). https://doi.org/10.1051/0004-6361/201935105, arXiv: 1901.07564

170. Moraux, E.: Open clusters and associations in the Gaia era. arXiv:1607.00027 (2016)

171. Mouret, S., Hestroffer, D., Mignard, F.: Asteroid masses and improvement with Gaia. A\&A 472, 1017-1027 (2007). https://doi.org/10.1051/0004-6361:20077479

172. Mowlavi, N., Lecoeur-Taïbi, I., Lebzelter, T., Rimoldini, L., Lorenz, D., Audard, M., De Ridder, J., Eyer, L., Guy, L.P., Holl, B.: Gaia Data Release 2. The first Gaia catalogue of longperiod variable candidates. A\&A 618, A58 (2018). https://doi.org/10.1051/0004-6361/201833366, arXiv: 1805.02035

173. Myeong, G.C., Vasiliev, E., Iorio, G., Evans, N.W., Belokurov, V.: Evidence for Two Early Accretion Events That Built the Milky Way Stellar Halo. arXiv:1904.03185 (2019) 
174. Nataf, D.M., Udalski, A., Gould, A., Fouqué, P., Stanek, K.Z.: The Split Red Clump of the Galactic Bulge from OGLE-III. ApJ 721, L28-L32 (2010). https://doi.org/10.1088/2041-8205/721/1/L28, arXiv: 1007.5065

175. Nelan, E.: Astrometry with the James Webb Space Telescope. In: Seidelmann, P.K., Monet, A.K.B. (eds.) Astrometry in the Age of the Next Generation of Large Telescopes, Astronomical Society of the Pacific Conference Series, vol. 338, p. 59 (2005)

176. Ness, M., Hogg, D.W., Rix, H.W., Ho, A.Y.Q., Zasowski, G.: The Cannon: A data-driven approach to Stellar Label Determination. ApJ 808(1), 16 (2015). https://doi.org/10.1088/0004-637X/808/1/16, arXiv: 1501.07604

177. Oelkers, R.J., Stassun, K.G., Dhital, S.: Gaia Assorted Mass Binaries Long Excluded from SLoWPoKES (GAMBLES): Identifying Ultra-wide Binary Pairs with Components of Diverse Mass. AJ 153(6), 259 (2017). https://doi.org/10.3847/1538-3881/aa6d55, arXiv:1611.07883

178. Oh, S., Price-Whelan, A.M., Hogg, D.W., Morton, T.D., Spergel, D.N.: Comoving Stars in Gaia DR1: An Abundance of Very Wide Separation Comoving Pairs. AJ 153(6), 257 (2017). https://doi.org/10.3847/1538-3881/aa6ffd, arXiv:1612.02440

179. Paczynski, B.: Gravitational microlensing by the galactic halo. ApJ 304, 1-5 (1986). https://doi. org/10.1086/164140

180. Perryman, M., Hartman, J., Bakos, G.Á., Lindegren, L.: Astrometric Exoplanet Detection with Gaia. ApJ 797, 14 (2014). https://doi.org/10.1088/0004-637X/797/1/14, arXiv:1411.1173

181. Perryman, M.A.C., de Boer, K.S., Gilmore, G., Høg, E., Lattanzi, M.G., Lindegren, L., Luri, X., Mignard, F., Pace, O., de Zeeuw, P.T.: GAIA: Composition, formation and evolution of the Galaxy. A\&A 369, 339-363 (2001). https://doi.org/10.1051/0004-6361:20010085, arXiv:astro-ph/0101235

182. Piffl, T., Binney, J., McMillan, P.J., Steinmetz, M., Helmi, A., Wyse, R.F.G., Bienaymé, O., BlandHawthorn, J., Freeman, K., Gibson, B., Gilmore, G., Grebel, E.K., Kordopatis, G., Navarro, J.F., Parker, Q., Reid, W.A., Seabroke, G., Siebert, A., Watson, F., Zwitter, T.: Constraining the Galaxy's dark halo with RAVE stars. MNRAS 445(3), 3133-3151 (2014). https://doi.org/10.1093/mnras/ stu1948, arXiv:1406.4130

183. Pittordis, C., Sutherland, W.: Testing modified-gravity theories via wide binaries and GAIA. MNRAS 480(2), 1778-1795 (2018). https://doi.org/10.1093/mnras/sty1578, arXiv:1711.10867

184. Pittordis, C., Sutherland, W.: Testing modified gravity with wide binaries in Gaia DR2. MNRAS 488(4), 4740-4752 (2019). https://doi.org/10.1093/mnras/stz1898, arXiv:1905.09619

185. Planck Collaboration, Aghanim, N., Armitage-Caplan, C., Arnaud, M., Ashdown, M., AtrioBarandela, F., Aumont, J., Baccigalupi, C., Banday, A.J., Barreiro, R.B., Bartlett, J.G., Benabed, K., Benoit-Lévy, A., Bernard, J.P., Bersanelli, M., Bielewicz, P., Bobin, J., Bock, J.J., Bond, J.R., Borrill, J., Bouchet, F.R., Bridges, M., Burigana, C., Butler, R.C., Cardoso, J.F., Catalano, A., Challinor, A., Chamballu, A., Chiang, H.C., Chiang, L.Y., Christensen, P.R., Clements, D.L., Colombo, L.P.L., Couchot, F., Crill, B.P., Curto, A., Cuttaia, F., Danese, L., Davies, R.D., Davis, R.J., de Bernardis, P., de Rosa, A., de Zotti, G., Delabrouille, J., Diego, J.M., Donzelli, S., Doré, O., Dupac, X., Efstathiou, G., Enßlin, T.A., Eriksen, H.K., Finelli, F., Forni, O., Frailis, M., Franceschi, E., Galeotta, S., Ganga, K., Giard, M., Giardino, G., González-Nuevo, J., Górski, K.M., Gratton, S., Gregorio, A., Gruppuso, A., Hansen, F.K., Hanson, D., Harrison, D.L., Helou, G., Hildebrandt, S.R., Hivon, E., Hobson, M., Holmes, W.A., Hovest, W., Huffenberger, K.M., Jones, W.C., Juvela, M., Keihänen, E., Keskitalo, R., Kisner, T.S., Knoche, J., Knox, L., Kunz, M., Kurki-Suonio, H., Lähteenmäki, A., Lamarre, J.M., Lasenby, A., Laureijs, R.J., Lawrence, C.R., Leonardi, R., Lewis, A., Liguori, M., Lilje, P.B., Linden-Vørnle, M., López-Caniego, M., Lubin, P.M., Macías-Pérez, J.F., Mandolesi, N., Maris, M., Marshall, D.J., Martin, P.G., Martínez-González, E., Masi, S., Massardi, M., Matarrese, S., Mazzotta, P., Meinhold, P.R., Melchiorri, A., Mendes, L., Migliaccio, M., Mitra, S., Moneti, A., Montier, L., Morgante, G., Mortlock, D., Moss, A., Munshi, D., Naselsky, P., Nati, F., Natoli, P., Nørgaard-Nielsen, H.U., Noviello, F., Novikov, D., Novikov, I., Osborne, S., Oxborrow, C.A., Pagano, L., Pajot, F., Paoletti, D., Pasian, F., Patanchon, G., Perdereau, O., Perrotta, F., Piacentini, F., Pierpaoli, E., Pietrobon, D., Plaszczynski, S., Pointecouteau, E., Polenta, G., Ponthieu, N., Popa, L., Pratt, G.W., Prézeau, G., Puget, J.L., Rachen, J.P., Reach, W.T., Reinecke, M., Ricciardi, S., Riller, T., Ristorcelli, I., Rocha, G., Rosset, C., Rubiño-Martín, J.A., Rusholme, B., Santos, D., Savini, G., Scott, D., Seiffert, M.D., Shellard, E.P.S., Spencer, L.D., Sunyaev, R., Sureau, F., Suur-Uski, A.S., Sygnet, J.F., Tauber, J.A., Tavagnacco, D., Terenzi, L., Toffolatti, L., Tomasi, M., Tristram, M., Tucci, M., Türler, M., Valenziano, L., Valiviita, J., Van Tent, B., Vielva, P., Villa, F., Vittorio, N., Wade, L.A., Wandelt, B.D., White, M., Yvon, D., Zacchei, A., Zibin, J.P., Zonca, A.: 
Planck 2013 results. XXVII. Doppler boosting of the CMB: Eppur si muove. A\&A 571, A27 (2014). https://doi.org/10.1051/0004-6361/201321556, arXiv:1303.5087

186. Poggio, E., Drimmel, R., Lattanzi, M.G., Smart, R.L., Spagna, A., Andrae, R., Bailer-Jones, C.A.L., Fouesneau, M., Antoja, T., Babusiaux, C., Evans, D.W., Figueras, F., Katz, D., Reylé, C., Robin, A.C., Romero-Gómez, M., Seabroke, G.M.: The Galactic warp revealed by Gaia DR2 kinematics. MNRAS 481(1), L21-L25 (2018). https://doi.org/10.1093/mnrasl/sly148, arXiv:1805.03171

187. Portail, M., Wegg, C., Gerhard, O., Martinez-Valpuesta, I.: Made-to-measure models of the Galactic box/peanut bulge: stellar and total mass in the bulge region. MNRAS 448, 713-731 (2015). https://doi.org/10.1093/mnras/stv058, arXiv:1502.00633

188. Posti, L., Helmi, A.: Mass and shape of the Milky Way's dark matter halo with globular clusters from Gaia and Hubble. A\&A 621, A56 (2019). https://doi.org/10.1051/0004-6361/201833355, arXiv: 1805.01408

189. Price-Whelan, A.M., Bonaca, A.: Off the Beaten Path: Gaia Reveals GD-1 Stars outside of the Main Stream. ApJ 863(2), L20 (2018). https://doi.org/10.3847/2041-8213/aad7b5, arXiv:1805.00425

190. Pyne, T., Gwinn, C.R., Birkinshaw, M., Eubanks, T.M., Matsakis, D.N.: Gravitational radiation and very long baseline interferometry. ApJ 465, 566 (1996). https://doi.org/10.1086/177443, arXiv:astro-ph/9507030

191. Queiroz, A.B.A., Anders, F., Santiago, B.X., Chiappini, C., Steinmetz, M., Dal Ponte, M., Stassun, K.G., da Costa, L.N., Maia, M.A.G., Crestani, J., Beers, T.C., Fernández-Trincado, J.G., García-Hernández, D.A., Roman-Lopes, A., Zamora, O.: StarHorse: a Bayesian tool for determining stellar masses, ages, distances, and extinctions for field stars. MNRAS 476(2), 2556-2583 (2018). https://doi.org/10.1093/mnras/sty330, arXiv:1710.09970

192. Queiroz, A.B.A., Anders, F., Chiappini, C., Khalatyan, A., Santiago, B.X., Steinmetz, M., Valentini, M., Miglio, A., Bossini, D., Barbuy, B., Minchev, I., Minniti, D., García Hernández, D.A., Schultheis, M., Beaton, R.L., Beers, T.C., Bizyaev, D., Brownstein, J.R., Cunha, K., FernándezTrincado, J.G., Frinchaboy, P.M., Lane, R.R., Majewski, S.R., Nataf, D., Nitschelm, C., Pan, K., Roman-Lopes, A., Sobeck, J.S., Stringfellow, G., Zamora, O.: From the bulge to the outer disc: StarHorse stellar parameters, distances, and extinctions for stars in APOGEE DR16 and other spectroscopic surveys. A\&A 638, A76 (2020). https://doi.org/10.1051/0004-6361/201937364, arXiv: 1912.09778

193. Quercellini, C., Cabella, P., Amendola, L., Quartin, M., Balbi, A.: Cosmic parallax as a probe of late time anisotropic expansion. Phys. Rev. D 80(6), 063527 (2009). https://doi.org/10.1103/PhysRevD. 80.063527, arXiv:0905.4853

194. Quercellini, C., Amendola, L., Balbi, A., Cabella, P., Quartin, M.: Real-time cosmology. Phys. Rep. 521, 95-134 (2012). https://doi.org/10.1016/j.physrep.2012.09.002, arXiv:1011.2646

195. Ranalli, P., Hobbs, D., Lindegren, L.: Astrometry and exoplanets in the Gaia era: a Bayesian approach to detection and parameter recovery. A\&A 614, A30 (2018). https://doi.org/10.1051/00046361/201730921, arXiv:1704.02493

196. Rauer, H., Catala, C., Aerts, C., Appourchaux, T., Benz, W., Brandeker, A., Christensen-Dalsgaard, J., Deleuil, M., Gizon, L., Goupil, M.J., Güdel, M., Janot-Pacheco, E., Mas-Hesse, M., Pagano, I., Piotto, G., Pollacco, D., Santos, C., Smith, A., Suárez, J.C., Szabó, R., Udry, S., Adibekyan, V., Alibert, Y., Almenara, J.M., Amaro-Seoane, P., Eiff, M.Av., Asplund, M., Antonello, E., Barnes, S., Baudin, F., Belkacem, K., Bergemann, M., Bihain, G., Birch, A.C., Bonfils, X., Boisse, I., Bonomo, A.S., Borsa, F., Brand ão, I.M., Brocato, E., Brun, S., Burleigh, M., Burston, R., Cabrera, J., Cassisi, S., Chaplin, W., Charpinet, S., Chiappini, C., Church, R.P., Csizmadia, S., Cunha, M., Damasso, M., Davies, M.B., Deeg, H.J., Díaz, R.F., Dreizler, S., Dreyer, C., Eggenberger, P., Ehrenreich, D., Eigmüller, P., Erikson, A., Farmer, R., Feltzing, S., de Oliveira Fialho, F., Figueira, P., Forveille, T., Fridlund, M., Garcí, R.A., Giommi, P., Giuffrida, G., Godolt, M., Gomes da Silva, J., Granzer, T., Grenfell, J.L., Grotsch-Noels, A., Günther, E., Haswell, C.A., Hatzes, A.P., Hébrard, G., Hekker, S., Helled, R., Heng, K., Jenkins, J.M., Johansen, A., Khodachenko, M.L., Kislyakova, K.G., Kley, W., Kolb, U., Krivova, N., Kupka, F., Lammer, H., Lanza, A.F., Lebreton, Y., Magrin, D., Marcos-Arenal, P., Marrese, P.M., Marques, J.P., Martins, J., Mathis, S., Mathur, S., Messina, S., Miglio, A., Montalban, J., Montalto, M., Monteiro, M.J.P.F.G., Moradi, H., Moravveji, E., Mordasini, C., Morel, T., Mortier, A., Nascimbeni, V., Nelson, R.P., Nielsen, M.B., Noack, L., Norton, A.J., Ofir, A., Oshagh, M., Ouazzani, R.M., Pápics, P., Parro, V.C., Petit, P., Plez, B., Poretti, E., Quirrenbach, A., Ragazzoni, R., Raimondo, G., Rainer, M., Reese, D.R., Redmer, R., Reffert, S., Rojas-Ayala, B., Roxburgh, I.W., Salmon, S., Santerne, A., Schneider, J., Schou, J., Schuh, S., 
Schunker, H., Silva-Valio, A., Silvotti, R., Skillen, I., Snellen, I., Sohl, F., Sousa, S.G., Sozzetti, A., Stello, D., Strassmeier, K.G., Švanda, M., Szabó, G.M., Tkachenko, A., Valencia, D., Van Grootel, V., Vauclair, S.D., Ventura, P., Wagner, F.W., Walton, N.A., Weingrill, J., Werner, S.C., Wheatley, P.J., Zwintz, K.: The PLATO 2.0 mission. Experiment. Astronom. 38(1-2), 249-330 (2014). https://doi.org/10.1007/s10686-014-9383-4, arXiv:1310.0696

197. Reid, M.J., Menten, K.M., Brunthaler, A., Zheng, X.W., Dame, T.M., Xu, Y., Wu, Y., Zhang, B., Sanna, A., Sato, M., Hachisuka, K., Choi, Y.K., Immer, K., Moscadelli, L., Rygl, K.L.J., Bartkiewicz, A.: Trigonometric Parallaxes of High Mass Star Forming Regions: The Structure and Kinematics of the Milky Way. ApJ 783, 130 (2014). https://doi.org/10.1088/0004-637X/783/2/130, arXiv: 1401.5377

198. Renzini, A., Gennaro, M., Zoccali, M., Brown, T.M., Anderson, J., Minniti, D., Sahu, K.C., Valenti, E., VandenBerg, D.A.: The WFC3 galactic bulge treasury program: Relative ages of bulge stars of high and low metallicity. Astrophysic. J. 863(1), 16 (2018). https://doi.org/10.3847/1538-4357/ aad09b

199. Ricci, L., Testi, L., Natta, A., Scholz, A., de Gregorio-Monsalvo, I.: ALMA observations of $\rho$-Oph 102: Grain growth and molecular gas in the disk around a young brown dwarf. ApJ 761(2), L20 (2012). https://doi.org/10.1088/2041-8205/761/2/L20, arXiv:1211.6743

200. Ricker, G.R., Winn, J.N., Vanderspek, R., Latham, D.W., Bakos, G.Á., Bean, J.L., Berta-Thompson, Z.K., Brown, T.M., Buchhave, L., Butler, N.R.: Transiting Exoplanet Survey Satellite (TESS). In: Proc. SPIE, Society of Photo-Optical Instrumentation Engineers (SPIE) Conference Series, vol. 9143, p. 914320 (2014). https://doi.org/10.1117/12.2063489, arXiv:1406.0151

201. Riess, A.G., Macri, L.M., Hoffmann, S.L., Scolnic, D., Casertano, S., Filippenko, A.V., Tucker, B.E., Reid, M.J., Jones, D.O., Silverman, J.M., Chornock, R., Challis, P., Yuan, W., Brown, P.J., Foley, R.J.: A 2.4Determination of the Local Value of the Hubble Constant. ApJ 826, 56 (2016). https://doi.org/10.3847/0004-637X/826/1/56, arXiv:1604.01424

202. Rizzi, L., Tully, R.B., Makarov, D., Makarova, L., Dolphin, A.E., Sakai, S., Shaya, E.J.: Tip of the Red Giant Branch Distances. II. Zero-Point Calibration. ApJ 661, 815-829 (2007). https://doi.org/10.1086/516566, arXiv:astro-ph/0701518

203. Roškar, R., Debattista, V.P., Quinn, T.R., Stinson, G.S., Wadsley, J.: Riding the spiral waves: Implications of stellar migration for the properties of galactic disks. ApJ 684, L79 (2008). https://doi.org/10.1086/592231, arXiv:0808.0206

204. Rybizki, J., Demleitner, M., Fouesneau, M., Bailer-Jones, C., Rix, H.W., Andrae, R.: A Gaia DR2 mock stellar catalog. PASP 130(7), 074101 (2018). https://doi.org/10.1088/1538-3873/aabd70, 1804.01427

205. Sahlmann, J., Nelan, E.G., Chayer, P., McLean, B., Lallo, M.: Optimisation of jwst operations with the help of gaia. Proc. Int. Astronomic. Union 12(S330), 98-99 (2017). https://doi.org/10.1017/S174 3921317006275

206. Sanders, J.L., Bovy, J., Erkal, D.: Dynamics of stream-subhalo interactions. MNRAS 457, 38173835 (2016). https://doi.org/10.1093/mnras/stw232, arXiv:1510.03426

207. Sanders, J.L., Smith, L., Evans, N.W.: The pattern speed of the Milky Way bar from transverse velocities. arXiv:1903.02009 (2019a)

208. Sanders, J.L., Smith, L., Evans, N.W., Lucas, P.: Transverse kinematics of the Galactic bar-bulge from VVV and Gaia. arXiv:1903.02008 (2019b)

209. Schönrich, R., Binney, J.: Origin and structure of the Galactic disc(s). MNRAS 399, 1145-1156 (2009). https://doi.org/10.1111/j.1365-2966.2009.15365.x, arXiv:0907.1899

210. Sellwood, J.A.: The lifetimes of spiral patterns in disc galaxies. MNRAS 410(3), 1637-1646 (2011). https://doi.org/10.1111/j.1365-2966.2010.17545.x, arXiv:1008.2737

211. Sellwood, J.A., Binney, J.J.: Radial mixing in galactic discs. MNRAS 336, 785-796 (2002). https://doi.org/10.1046/j.1365-8711.2002.05806.x, arXiv:astro-ph/0203510

212. Sharma, S., Bland-Hawthorn, J., Johnston, K.V., Binney, J.: Galaxia: A code to generate a synthetic survey of the Milky Way. ApJ 730, 3 (2011). https://doi.org/10.1088/0004-637X/730/1/3, arXiv: 1101.3561

213. Sim, G., Lee, S.H., Ann, H.B., Kim, S.: Open cluster survey within 1 kpc by the Gaia DR2. arXiv:1907.06872 (2019)

214. Simon, J.D.: Gaia proper motions and orbits of the ultra-faint milky way satellites. Astrophysic. J. 863(1), 89 (2018). https://doi.org/10.3847/1538-4357/aacdfb 
215. Simpson, J.D., Martell, S.L., Da Costa, G., Casey, A.R., Freeman, K.C., Horner, J., Ting, Y.S., Nataf, D.M., Lewis, G.F., Ness, M.K., Zucker, D.B., Cottrell, P.L., Čotar, K., Asplund, M., BlandHawthorn, J., Buder, S., D’Orazi, V., De Silva, G.M., Duong, L., Kos, J., Lin, J., Lind, K., Schlesinger, K.J., Sharma, S., Zwitter, T., Kafle, P.R., Nordlander, T.: The GALAH survey: coorbiting stars and chemical tagging. MNRAS 482(4), 5302-5315 (2019). https://doi.org/10.1093/ mnras/sty3042, arXiv:1804.05894

216. Smart, R.L., Marocco, F., Caballero, J.A., Jones, H.R.A., Barrado, D., Beamín, J.C., Pinfield, D.J., Sarro, L.M.: The Gaia ultracool dwarf sample - I. Known L and T dwarfs and the first Gaia data release. MNRAS 469(1), 401-415 (2017). https://doi.org/10.1093/mnras/stx800, arXiv:1703.09454

217. Smart, R.L., Marocco, F., Sarro, L.M., Barrado, D., Beamín, J.C., Caballero, J.A., Jones, H.R.A.: The Gaia ultracool dwarf sample - II. Structure at the end of the main sequence. MNRAS 485(3), 4423-4440 (2019). https://doi.org/10.1093/mnras/stz678, arXiv:1902.07571

218. Smith, L.C., Lucas, P.W., Kurtev, R., Smart, R., Minniti, D., Borissova, J., Jones, H.R.A., Zhang, Z.H., Marocco, F., Contreras Peña, C., Gromadzki, M., Kuhn, M.A., Drew, J.E., Pinfield, D.J., Bedin, L.R.: VIRAC: the VVV Infrared Astrometric Catalogue. MNRAS 474(2), 1826-1849 (2018). https://doi.org/10.1093/mnras/stx2789, arXiv:1710.08919

219. Solway, M., Sellwood, J.A., Schönrich, R.: Radial migration in galactic thick discs. MNRAS 422(2), 1363-1383 (2012). https://doi.org/10.1111/j.1365-2966.2012.20712.x, arXiv:1202.1418

220. Sovers, O.J., Fanselow, J.L., Jacobs, C.S.: Astrometry and geodesy with radio interferometry: experiments, models, results. Rev. Modern Phys. 70, 1393-1454 (1998). https://doi.org/10.1103/ RevModPhys.70.1393, arXiv:astro-ph/9712238

221. Sozzetti, A.: Astrometric methods and instrumentation to identify and characterize Extrasolar Planets: A Review. PASP 117(836), 1021-1048 (2005). https://doi.org/10.1086/444487, arXiv:astro-ph/0507115

222. Sozzetti, A.: Gaia, non-single stars, brown dwarfs, and exoplanets. Mem. Soc. Astron. Italiana 85, 643 (2014). arXiv: 1406.1388

223. Sozzetti, A., de Bruijne, J.: Space Astrometry Missions for Exoplanet Science: Gaia and the Legacy of Hipparcos, p. 81. Springer, Berlin (2018). https://doi.org/10.1007/978-3-319-55333-7_81

224. Sozzetti, A., Casertano, S., Lattanzi, M.G., Spagna, A.: Detection and measurement of planetary systems with GAIA. A\&A 373, L21-L24 (2001). https://doi.org/10.1051/0004-6361:20010788, arXiv:astro-ph/0104391

225. Sozzetti, A., Giacobbe, P., Lattanzi, M.G., Micela, G., Morbidelli, R., Tinetti, G.: Astrometric detection of giant planets around nearby M dwarfs: the Gaia potential. MNRAS 437(1), 497-509 (2014). https://doi.org/10.1093/mnras/stt1899, arXiv:1310.1405

226. Spergel, D., Gehrels, N., Baltay, C., Bennett, D., Breckinridge, J., Donahue, M., Dressler, A., Gaudi, B.S., Greene, T., Guyon, O.: Wide-Field InfrarRed Survey Telescope-Astrophysics Focused Telescope Assets WFIRST-AFTA 2015 Report. arXiv:1503.03757 (2015)

227. Spoto, F., Milani, A., Knežević, Z.: Asteroid family ages. Icarus 257, 275-289 (2015). https://doi.org/10.1016/j.icarus.2015.04.041, arXiv:1504.05461

228. Standish, E.M., Fienga, A.: Accuracy limit of modern ephemerides imposed by the uncertainties in asteroid masses. A\&A 384, 322-328 (2002). https://doi.org/10.1051/0004-6361:20011821

229. Takada, M., Ellis, R.S., Chiba, M., Greene, J.E., Aihara, H., Arimoto, N., Bundy, K., Cohen, J., Doré, O., Graves, G., Gunn, J.E., Heckman, T., Hirata, C.M., Ho, P., Kneib, J.P., Le Fèvre, O., Lin, L., More, S., Murayama, H., Nagao, T., Ouchi, M., Seiffert, M., Silverman, J.D., Sodré, L., Spergel, D.N., Strauss, M.A., Sugai, H., Suto, Y., Takami, H., Wyse, R.: Extragalactic science, cosmology, and Galactic archaeology with the Subaru Prime Focus Spectrograph. PASJ 66, R1 (2014). https://doi.org/10.1093/pasj/pst019, arXiv:1206.0737

230. Tanga, P., Delbó, M.: Asteroid occultations today and tomorrow: toward the GAIA era. A\&A 474, 1015-1022 (2007). https://doi.org/10.1051/0004-6361:20077470, arXiv:0801.2684

231. Taris, F., Souchay, J., Andrei, A.H., Bernard, M., Salabert, M., Bouquillon, S., Anton, S., Lambert, S.B., Gontier, A.M., Barache, C.: Astrophotometric variability of CFHT-LS Deep 2 QSOs. A\&A 526, A25 (2011). https://doi.org/10.1051/0004-6361/201015500

232. The MSE Science Team, Babusiaux, C., Bergemann, M., Burgasser, A., Ellison, S., Haggard, D., Huber, D., Kaplinghat, M., Li, T., Marshall, J., et al: The Detailed Science Case for the Maunakea Spectroscopic Explorer, 2019 edition. arXiv:1904.04907 (2019) 
233. Titov, O., Lambert, S.B., Gontier, A.M.: VLBI measurement of the secular aberration drift. A\&A 529, A91 (2011). https://doi.org/10.1051/0004-6361/201015718, arXiv:1009.3698

234. Tonry, J.L., Stubbs, C.W., Lykke, K.R., Doherty, P., Shivvers, I.S., Burgett, W.S., Chambers, K.C., Hodapp, K.W., Kaiser, N., Kudritzki, R.P.: The Pan-STARRS1 Photometric System. ApJ 750(2), 99 (2012). https://doi.org/10.1088/0004-637X/750/2/99, arXiv:1203.0297

235. Tremaine, S., Weinberg, M.D.: A kinematic method for measuring the pattern speed of barred galaxies. ApJ 282, L5-L7 (1984). https://doi.org/10.1086/184292

236. Trick, W.H., Bovy, J., D’Onghia, E., Rix, H.W.: Action-based dynamical modeling for the Milky Way Disk: The influence of spiral arms. ApJ 839(1), 61 (2017). https://doi.org/10.3847/1538-4357/aa67db, arXiv:1703.05970

237. Unwin, S.C., Shao, M., Tanner, A.M., Allen, R.J., Beichman, C.A., Boboltz, D., Catanzarite, J.H., Chaboyer, B.C., Ciardi, D.R., Edberg, S.J., Fey, A.L., Fischer, D.A., Gelino, C.R., Gould, A.P., Grillmair, C., Henry, T.J., Johnston, K.V., Johnston, K.J., Jones, D.L., Kulkarni, S.R., Law, N.M., Majewski, S.R., Makarov, V.V., Marcy, G.W., Meier, D.L., Olling, R.P., Pan, X., Patterson, R.J., Pitesky, J.E., Quirrenbach, A., Shaklan, S.B., Shaya, E.J., Strigari, L.E., Tomsick, J.A., Wehrle, A.E., Worthey, G.: Taking the measure of the Universe: Precision astrometry with sim planetquest. PASP 120, 38-88 (2008). https://doi.org/10.1086/525059, arXiv:0708.3953

238. Valenti, E., Ferraro, F.R., Origlia, L.: Red giant branch in near-infrared colour-magnitude diagrams - I. Calibration of photometric indices. MNRAS 351, 1204-1214 (2004a). https://doi.org/10.1111/j. 1365-2966.2004.07861.x, arXiv:astro-ph/0403563

239. Valenti, E., Ferraro, F.R., Origlia, L.: Red giant branch in near-infrared colour-magnitude diagrams - II. The luminosity of the bump and the tip. MNRAS 354, 815-820 (2004b). https://doi.org/10.1111/j.1365-2966.2004.08249.x, arXiv:astro-ph/0404403

240. Valenti, E., Zoccali, M., Gonzalez, O.A., Minniti, D., Alonso-Garcí, J., Marchetti, E., Hempel, M., Renzini, A., Rejkuba, M.: Stellar density profile and mass of the Milky Way bulge from VVV data. A\&A 587, L6 (2016). https://doi.org/10.1051/0004-6361/201527500, arXiv:1510.07425

241. Vallée, J.P.: A guided map to the spiral arms in the galactic disk of the Milky Way. The Astronomical Review 13(3-4), 113-146 (2017a). https://doi.org/10.1080/21672857.2017.1379459, arXiv: 1711.05228

242. Vallée, J.P.: Constraining the pitch angle of the galactic spiral arms in the Milky Way. New A Rev. 79, 49-58 (2017b). https://doi.org/10.1016/j.newar.2017.09.001, arXiv:1709.05303

243. Vokrouhlický, D., Brož, M., Bottke, W.F., Nesvorný, D., Morbidelli, A.: Yarkovsky/YORP chronology of asteroid families. Icarus 182, 118-142 (2006). https://doi.org/10.1016/j.icarus.2005.12.010

244. Watkins, L.L., van der Marel, R.P., Sohn, S.T., Evans, N.W.: Evidence for an Intermediatemass Milky Way from Gaia DR2 Halo Globular Cluster Motions. ApJ 873(2), 118 (2019). https://doi.org/10.3847/1538-4357/ab089f, arXiv:1804.11348

245. Wegg, C., Gerhard, O., Portail, M.: The structure of the Milky Way's bar outside the bulge. MNRAS 450, 4050-4069 (2015). https://doi.org/10.1093/mnras/stv745

246. Wegg, C., Gerhard, O., Bieth, M.: The gravitational force field of the Galaxy measured from the kinematics of RR Lyrae in Gaia. MNRAS 485(3), 3296-3316 (2019). https://doi.org/10.1093/mnras/stz572, arXiv:1806.09635

247. Windmark, F., Lindegren, L., Hobbs, D.: Using Galactic Cepheids to verify Gaia parallaxes. A\&A 530, A76 (2011). https://doi.org/10.1051/0004-6361/201116929, arXiv:1104.2348

248. Wyrzykowski, Ł., Kostrzewa-Rutkowska, Z., Skowron, J., Rybicki, K.A., Mróz, P., Kozłowski, S., Udalski, A., Szymański, M.K., Pietrzyński, G., Soszyński, I., Ulaczyk, K., Pietrukowicz, P., Poleski, R., Pawlak, M., Iłkiewicz, K., Rattenbury, N.J.: Black hole, neutron star and white dwarf candidates from microlensing with OGLE-III ${ }^{\star}$. MNRAS 458, 3012-3026 (2016). https://doi.org/10.1093/mnras/stw426, 1509.04899

249. Wyrzykowski, L., Mróz, P., Rybicki, K.A., Gromadzki, M., Kołaczkowski, Z., Zieliński, M., Zieliński, P., Britavskiy, N., Gomboc, A., Sokolovsky, K., Hodgkin, S.T., Abe, L., Aldi, G.F., AlMannaei, A., Altavilla, G., Al Qasim, A., Anupama, G.C., Awiphan, S., Bachelet, E., Bakiş, V., Baker, S., Bartlett, S., Bendjoya, P., Benson, K., Bikmaev, I.F., Birenbaum, G., Blagorodnova, N., BlancoCuaresma, S., Boeva, S., Bonanos, A.Z., Bozza, V., Bramich, D.M., Bruni, I., Burenin, R.A., Burgaz, U., Butterley, T., Caines, H.E., Caton, D.B., Calchi Novati, S., Carrasco, J.M., Cassan, A., Čepas, V., Cropper, M., Chruślińska, M., Clementini, G., Clerici, A., Conti, D., Conti, M., Cross, S., Cusano, F., Damljanovic, G., Dapergolas, A., D’Ago, G., de Bruijne, J.HJ., Dennefeld, M., Dhillon, V.S., Dominik, M., Dziedzic, J., Erece, O., Eselevich, M.V., Esenoglu, H., Eyer, L., Figuera Jaimes, R., 
Fossey, S.J., Galeev, A.I., Grebenev, S.A., Gupta, A.C., Gutaev, A.G., Hallakoun, N., Hamanowicz, A., Han, C., Handzlik, B., Haislip, J.B., Hanlon, L., Hardy, L.K., Harrison, D.L., van Heerden, H.J., Hoette, V.L., Horne, K., Hudec, R., Hundertmark, M., Ihanec, N., Irtuganov, E.N., Itoh, R., Iwanek, P., Jovanovic, M.D., Janulis, R., Jelínek, M., Jensen, E., Kaczmarek, Z., Katz, D., Khamitov, I.M., Kilic, Y., Klencki, J., Kolb, U., Kopacki, G., Kouprianov, V.V., Kruszyńska, K., Kurowski, S., Latev, G., Lee, C.-H., Leonini, S., Leto, G., Lewis, F., Li, Z., Liakos, A., Littlefair, S.P., Lu, J., Manser, C.J., Mao, S., Maoz, D., Martin-Carrillo, A., Marais, J.P., Maskoliūnas, M., Maund, J.R., Meintjes, P.J., Melnikov, S.S., Ment, K., Mikołajczyk, P., Morrell, M., Mowlavi, N., Moździerski, D., Murphy, D., Nazarov, S., Netzel, H., Nesci, R., Ngeow, C.-C., Norton, A.J., Ofek, E.O., Pakštienè, E., Palaversa, L., Pandey, A., Paraskeva, E., Pawlak, M., Penny, M.T., Penprase, B.E., Piascik, A., Prieto, J.L., Qvam, J.KT., Ranc, C., Rebassa-Mansergas, A., Reichart, D.E., Reig, P., Rhodes, L., Rivet, J.-P., Rixon, G., Roberts, D., Rosi, P., Russell, D.M., Zanmar Sanchez, R., Scarpetta, G., Seabroke, G., Shappee, B.J., Schmidt, R., Shvartzvald, Y., Sitek, M., Skowron, J., Śniegowska, M., Snodgrass, C., Soares, P.S., van Soelen, B., Spetsieri, Z.T., Stankevičiūè, A., Steele, I.A., Street, R.A., Strobl, J., Strubble, E., Szegedi, H., Tinjaca Ramirez, L.M., Tomasella, L., Tsapras, Y., Vernet, D., Villanueva, S., Vince, O., Wambsganss, J., van der Westhuizen, I.P., Wiersema, K., Wium, D., Wilson, R.W., Yoldas, A., Zhuchkov, R.Y.a., Zhukov, D.G., Zdanavičius, J., Zoła, S., Zubareva, A.: Full orbital solution for the binary system in the northern Galactic disc microlensing event Gaia16aye. A\&A 633, A98 (2020). https://doi.org/10.1051/0004-6361/201935097

250. Yano, T., Gouda, N., Kobayashi, Y., Tsujimoto, T., Niwa, Y., Yamada, Y.: The scientific goal of the Japanese small astrometric satellite, Small-JASMINE. In: de Grijs, R. (ed.) Advancing the Physics of Cosmic Distances, IAU Symposium, vol. 289, pp. $433-436$ (2013). https://doi.org/10.1017/S1743921312021898

251. Zhao, G., Zhao, Y., Chu, Y., Jing, Y., Deng, L.: LAMOST Spectral Survey. arXiv:1206.3569 (2012)

252. Zoccali, M., Valenti, E.: The 3D Structure of the Galactic Bulge. PASA 33, e025 (2016). https://doi.org/10.1017/pasa.2015.56, arXiv:1601.02839

253. Zwitter, T.: Galactic astronomy and small telescopes. Contributions of the Astronomical Observatory Skalnate Pleso 49, 320-329 (2019). arXiv:1812.06461

Publisher's note Springer Nature remains neutral with regard to jurisdictional claims in published maps and institutional affiliations. 


\section{Affiliations}

David Hobbs ${ }^{1}$ (1) . Anthony Brown ${ }^{2}$. Erik Høg ${ }^{3}$. Carme Jordi ${ }^{4}$.

Daisuke Kawata ${ }^{5}$. Paolo Tanga $^{6} \cdot$ Sergei Klioner $^{7}$. Alessandro Sozzetti ${ }^{8}$. Łukasz Wyrzykowski ${ }^{9}$. Nicholas Walton ${ }^{10}$. Antonella Vallenari ${ }^{11}$.

Valeri Makarov ${ }^{12}$. Jan Rybizki ${ }^{13}$. Fran Jiménez-Esteban ${ }^{14}$. José A. Caballero ${ }^{14}$. Paul J. McMillan ${ }^{1}$. Nathan Secrest ${ }^{12} \cdot$ Roger Mor $^{4}$. Jeff J. Andrews ${ }^{3}$.

Tomaž Zwitter $^{15}$. Cristina Chiappini ${ }^{16}$. Johan P. U. Fynbo ${ }^{3}$. Yuan-Sen Ting ${ }^{17}$. Daniel Hestroffer ${ }^{18}$. Lennart Lindegren ${ }^{1}$. Barbara McArthur ${ }^{19}$. Naoteru Gouda ${ }^{20}$ - Anna Moore ${ }^{21}$. Oscar A. Gonzalez ${ }^{22} \cdot$ Mattia Vaccari $^{23,24}$

1 Lund Observatory, Lund University, Box 43, SE-22100, Lund, Sweden

2 Leiden Observatory, Leiden University, Niels Bohrweg 2, 2333, CA, Leiden, The Netherlands

3 Niels Bohr Institute, University of Copenhagen, Blegdamsvej 17, 2100, Copenhagen, Denmark

4 Institut de Ciències del Cosmos, ICCUB-IEEC, Barcelona, Spain

5 Mullard Space Science Laboratory, University College London, Holmbury St. Mary, Dorking, RH5 6NT, UK

6 Observatoire de la Côte d'Azur, 06304, Nice, Cedex 4, France

7 Lohrmann Observatory, Technical University Dresden, Dresden, Germany

8 INAF-Osservatorio Astrofisico di Torino, Via Osservatorio 20, 10025, Pino Torinese, Italy

9 Warsaw University Observatory, Al. Ujazdowskie 4, 00-478, Warszawa, Poland

10 Institute of Astronomy, University of Cambridge, Madingley Road, Cambridge, CB3 OHA, UK

11 Osservatorio Astronomico di Padova, INAF, Vicolo dell'Osservatorio 5, I-35122, Padova, Italy

12 U.S. Naval Observatory, 3450 Massachusetts Avenue NW, Washington, DC, 20392, USA

13 Max Planck Institute for Astronomy, Königstuhl 17, D-69117, Heidelberg, Germany

14 Centro de Astrobiología (CSIC-INTA), Camino Bajo del Castillo s/n, 28692, Villanueva de la Cañada, Spain

15 University of Ljubljana, Faculty of Mathematics and Physics, Ljubljana, Slovenia

16 Leibniz-Institut für Astrophysik Potsdam (AIP), An der Sternwarte 16, 14482, Potsdam, Germany

17 Institute for Advanced Study, Princeton, NJ, 08540, USA

18 IMCCE - Observatoire de Paris, F-75014, Paris, France

19 McDonald Observatory, University of Texas at Austin, Austin, TX, 78712-1205, USA

20 National Astronomical Observatory of Japan, 2-21-1, Osawa, Mitaka, Tokyo, 181-8588, Japan

21 The Australian National University, Canberra, ACT 2611, Australia

22 STFC UK Astronomy Technology Centre, The Royal Observatory Edinburgh, EH9 3HJ, Edinburgh, $\mathrm{UK}$

23 Inter-university Institute for Data Intensive Astronomy and Department of Physics and Astronomy, University of the Western Cape, Robert Sobukwe Road, 7535, Bellville, Cape Town, South Africa

24 INAF - Istituto di Radioastronomia, via Gobetti 101, 40129, Bologna, Italy 